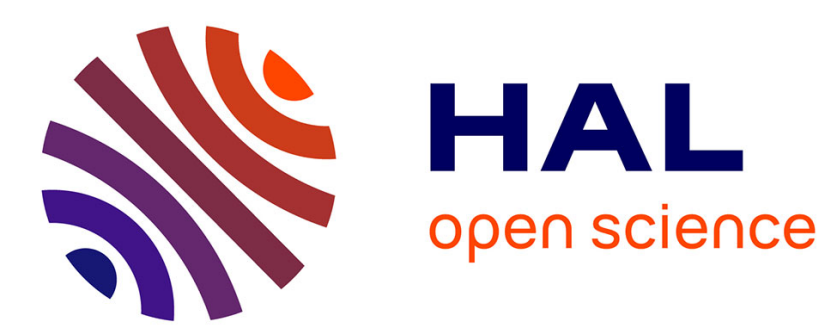

\title{
Coupling between oxidation kinetics and anisothermal oil flow during deep-fat frying
}

Maxime Touffet, Mohamed Hatem Allouche, Mostapha Ariane, Olivier Vitrac

\section{To cite this version:}

Maxime Touffet, Mohamed Hatem Allouche, Mostapha Ariane, Olivier Vitrac. Coupling between oxidation kinetics and anisothermal oil flow during deep-fat frying. 2021. hal-03210292

\section{HAL Id: hal-03210292 \\ https://hal.science/hal-03210292}

Preprint submitted on 27 Apr 2021

HAL is a multi-disciplinary open access archive for the deposit and dissemination of scientific research documents, whether they are published or not. The documents may come from teaching and research institutions in France or abroad, or from public or private research centers.
L'archive ouverte pluridisciplinaire HAL, est destinée au dépôt et à la diffusion de documents scientifiques de niveau recherche, publiés ou non, émanant des établissements d'enseignement et de recherche français ou étrangers, des laboratoires publics ou privés. 


\section{Coupling between oxidation kinetics and anisothermal oil}

3 Maxime Touffet ${ }^{\mathrm{a}}$, Mohamed Hatem Allouche ${ }^{\mathrm{b}}$, Mostapha Ariane ${ }^{\mathrm{b}}$, Olivier Vitrac ${ }^{\mathrm{c} *}$

${ }^{b}$ UMR 5209 Laboratoire Interdisciplinaire Carnot de Bourgogne, Université de Bourgogne-

6

\section{Franche Comté, Dijon, France}

${ }^{c}$ UMR 0782 SayFood Paris-Saclay Food and Bioproducts Engineering Research Unit, INRAE, AgroParisTech, Université Paris-Saclay, 91300 Massy, France

\section{Highlights}

- description of oil flow and temperature distribution in kitchen appliances during heating and holding times;

- simplified descriptions of the generation and decomposition of hydroperoxides from triacylglycerols as a mixture of fatty esters in frying conditions;

- coupling between Eulerian and Lagrangian descriptions to analyze the effects of residence times at different temperatures;

- validation experiments with a modified and instrumented deep-fryer and reference decomposition kinetics in anoxia.

\footnotetext{
* Corresponding author. Tel : +33169935063; fax: +33169935044; E-mail Address:
} olivier.vitrac@agroparistech.fr (O. Vitrac). 


\section{Abstract}

23 Deep-fat frying is a cooking technique that has been used continuously since prehistoric times.

24 A domestic deep-fryer heated from the bottom develops significant convection inside the bath cavity. It is responsible for very high heat transfer coefficients and the deep-frying oil exposure to the atmospheric oxygen. The continuous conversion of gaseous dioxygen into unstable and reactive hydroperoxides and their subsequent advection throughout the bulk volume is at the origin of the main complaints made of frying: odors, fouling, and generation of several toxic compounds. This study analyzes the coupling between natural convection of triacylglycerols and the combinatorial chemistry controlling their autoxidation. At macroscopic scale, anisothermal flow within a typical kitchen appliance, including a cold zone beneath the heating element, was simulated in 3D under the Boussinesq approximation with an Eulerian description during initial heating and temperature holding. At molecular and intermediate scales, the endothermic decomposition of fatty-ester hydroperoxides in mixtures was simulated over several hours using a Lagrangian description. Simulations and the proposed description of the combinatorial generation of hydroperoxides highlighted the complex relationship between deep-fryer design, residence times at different temperatures, and oil composition. The halflifetime of hydroperoxides $(>15 \mathrm{~min})$ is intermediate between vertical mixing times $(<3 \mathrm{~min})$ and longitudinal ones ( $>2$ hours). Hydroperoxides of unsaturated fatty esters are generated at the surface, are preferentially decomposed in heated plumes and accumulate between production cycles in cold stagnant regions. Experimental validation is proposed for the eddy structure, temperature fluctuations, and hydroperoxides decomposition kinetics.

\section{Keywords}

Heat and mass transfer, oil oxidation, CFD, anisothermal oil flow, frying 


\section{Introduction}

Deep-frying consists of immersing in a rich-water product in a liquid with a high boiling point liquid, usually a vegetable oil (denoted oil in the rest of the article). Oil is an ingredient incorporated within the finished product during cooling $[1,2]$ and the heat transfer fluid. Oil is a high Prandtl number fluid enabling intense heat transfer coefficients by natural convection (i.e., in the vertical direction). As a result, fried products (French-fries, potato chips, chicken pieces and fingers, fish fillets, shrimp) are hot and crispy on the outside and cooked safely in the center (low risk of residual bacteria contamination). Due to the cycle of production of fried products in kitchen appliances, the residence time of oil within batch deep-fryers (several hours after repeated heating cycles) is much longer than frying time (indicatively from five to twenty minutes). Oil abuse by thermo-oxidation due to oxygen exposure at high temperatures can lead to various toxic compounds, with possible health concerns [3]. A similar matter exists for Maillard reactions with the apparition of carcinogenic acrylamide in contact with oil at high temperature [4]. Though no regulations have been established in the United States, the apparition of oxidation products is strongly regulated in the EU [5].

Vegetable oils are mixtures of triacylglycerols (TAG), which can be described as three fatty acids esterified onto the same glycerol backbone. Fatty esters (FE) naturally exhibit a variable number of carbon-carbon double bonds (unsaturations), which depend on plan variety [6]. Prevalent mono-, di-, and tri- unsaturated FE are the oleate (C18:1), linoleate (C18:2), and linolenate (C18:3). Unsaturated fatty esters are desirable in the diet with the following preference ratios 1:4:1 for FE with unsaturation in the $n-6, n-3, n-9$ position (also called $\omega-6$, $\omega-3, \omega-9$ FE), respectively $[7,8]$. The positions are counted from the methyl end. These unsaturations are brought in common frying oils by linoleate $(n-6)$, linolenate $(n-3)$, and oleate (n-9), respectively. For example, these three fatty esters represent $c a .90 \%$ of FE in sunflower 
oil. They are also the most sensitive to thermo-oxidation. The complex chemistry of thermooxidation leads to a dramatic loss of nutritional value by a displacement of double bonds (conjugated hydroperoxides), the apparition scission products (e.g., volatile aldehydes and esters [9]) responsible for off-flavors and toxic compounds (e.g., 4-HNE [3]), the production of cross-linked polymers responsible of fouling.

The first stages of the thermo-oxidation process are controlled by the kinetics of oxygen dissolution and intermediary products' transport through the oil bath [10, 11]. Similar effects have been shown in thermoplastics, where oxidation and polymer damages start from the external surfaces and the most exposed to atmospheric oxygen [12]. In simple words, mineral oxygen from the atmosphere is converted at the oil-air interface into chemically bounded oxygen (hydroperoxides). Hydroperoxides are thermally unstable products whose endothermic decomposition (i.e., activated by increasing temperatures) generate either scission products (products responsible for the typical smell of fried products) or alkyl radicals, very reactive with oxygen. When the latter dominate, the oxidation process propagate in chain between the surface, the bulk, and the heating elements. In most of the EU member states, it is considered by law that frying oil should be discarded as soon as total polar compounds (TPC) exceed $25 \%$ in mass or a value similar. Polar compounds are defined as any species incorporating oxygen atoms beyond the initially present esters. Therefore, the legal oil lifetime depends on the rate of renewal of radicals responsible for the production of hydroperoxides and their subsequent decomposition in hot regions. As fried foods represent a significant fraction of the diet in Western and Asian countries, there is an urgent need to identify the possible strategies to minimize oil abuse and reduce oil wastes. Prior research focused on the reduction of oil uptake in fried products $[1,2]$. While improving the dietary balance, this strategy reduces consumer exposure to oxidized products but does not explicitly address the loss of nutritional value in fried products. Little work has been done to relate the design of batch deep-fryers with oxidation 
kinetics. Pioneer works $[13,14]$ in the sixties showed that substantial gain could be achieved by updating the surface-to-volume ratio of oil and oil flow distribution in the bath.

The current study addresses the lifetime hydroperoxides transported by the natural convection in the cavity of a kitchen appliance deep-fryer. We chose as reference a deep-fryer design including a cold zone below the submerged heating electrical resistor. Their decomposition is highly endothermic and is the root cause for all the inconveniences encountered during deepfrying: fouling, bad smells, reduced lifetimes, the apparition of toxic compounds. Coupling an anisothermal flow with a chemical reaction requires special treatment, as the molecular carrier is a TAG carrying different chemical functions with variable sensitivity to oxidation. This work shows as a Lagrangian description can reconcile the models based on mass balance on chemical functions and transport by an anisothermal flow.

The paper is organized as follows. Since deep-frying involves temperatures above the boiling point of pure water, section 2 justifies and details the oxidation model of edible oil between $80^{\circ} \mathrm{C}$ and $180^{\circ} \mathrm{C}$ and the formulation of hydroperoxide decomposition along streamlines. Our simulations cover the heating and holding periods, which is $50 \%$ to $90 \%$ of the deep-fryer's operation time. Indeed, the heat flux density between the oil and the frying product is so high (up to $150 \mathrm{~kW} \cdot \mathrm{m}^{-2}[15,16]$ ) that a batch deep-fryer cannot operate a steady-state. Most of the operation time is used to recover the set temperature (maximum temperature $175^{\circ} \mathrm{C}$ according to [5]). The experimental conditions used to parameterize the hydroperoxides' decomposition model in isothermal conditions and to validate it in anisothermal ones are detailed in Section 3. Simulated results and the coupling between the transport and the degradation are analyzed in Section 4. Finally, the last section summarizes the main findings and the directions to keep oil healthy during deep-frying. 
118

\section{NUMERICAL METHODS}

\section{A. Combinatorial oxidation reaction kinetics}

\section{Principles}

Triplet dioxygen is the common allotrope of oxygen in our environment. Because it carries two unpaired electrons, occupying two $\pi$ molecular orbitals, it is a stable diradical, denoted ${ }^{\bullet} \mathrm{OO}^{\bullet}$ throughout the manuscript, as justified in Ref. [17]. Its unusual electronic structure explains why this abundant molecule is encouraged to react in the deep-fryer with an alkyl radical $\mathrm{L}^{\bullet}$ centered on the carbon by forming bonds. Both radicals (di- and mono-) stabilize themselves, but the extra valence of dioxygen creates a new radical centered on oxygen, so-called peroxyl radicals, and denoted $\mathrm{LOO}^{\bullet}$. At this stage, it is crucial noticing that the abundant ${ }^{\bullet} \mathrm{OO}^{\bullet}$ diradicals cannot directly attack a fatty ester LH. The attack involves an intermediate step of hydrogen abstraction between $\mathrm{LOO}^{\circ}$ and $\mathrm{LH}$ (reaction $R_{05}$ in Eq. (1)). This step between a radical and a stable species preserves free-valence and propagates it from oxygen to carbon atoms. The first oxidation products of fatty esters are hydroperoxides LOOH and radicals $\mathrm{L}^{\bullet}$. In the context of deep-frying, the chain reaction's initiation occurs mainly by the continuous regeneration of $\mathrm{L}^{\bullet}$ radicals at high temperatures [11]. Hydroperoxides are, indeed, unstable above $100^{\circ} \mathrm{C}$ [18] and decompose endothermically by homolytic scission of LO-OH bond (bond energy between 100 and $160 \mathrm{~kJ} \cdot \mathrm{mol}^{-1}$ ) [19].

Bolland and Tobolsky [18, 20-22] pioneered the first general kinetic description of hydroperoxides in unsaturated materials (lipids, thermoplastic polymers). Depending on whether hydrogen bonds stabilize a cage mechanism (see Ref. [23]) or not, their decompositions are considered either unimolecular [24-35] or bimolecular [31, 33, 35, 36]. The molecularity of the decomposition remains an open question (see the discussion in Ref. [11]) in frying 
141 conditions, but first-order decay has been mainly observed [24-26, 37-40]. In this work,

142 unimolecular decomposition was chosen accordingly. The detailed mechanism reads [19, 30,

143 41]:

$$
\begin{aligned}
& \mathrm{R}_{01}: \mathrm{LOOH}^{\stackrel{\mathrm{k}_{\mathrm{d}}}{\longrightarrow}} \mathrm{LO}^{\bullet}+{ }^{\bullet} \mathrm{OH} \\
& \mathrm{R}_{02}: \mathrm{LO}^{\bullet}+\mathrm{LH} \stackrel{\mathrm{k}_{\mathrm{b}}}{\longrightarrow} \mathrm{LOH}+\mathrm{L}^{\bullet} \\
& \mathrm{R}_{03}: \mathrm{HO}^{\bullet}+\mathrm{LH} \stackrel{\mathrm{k}_{\mathrm{b}}^{\prime}}{\longrightarrow} \mathrm{H}_{2} \mathrm{O}+\mathrm{L}^{\bullet} \\
& \mathrm{R}_{04}: \mathrm{L}^{\bullet}+{ }^{\bullet} \mathrm{OO} \stackrel{\mathrm{k}_{\mathrm{o}}}{\longrightarrow} \mathrm{LOO}^{\bullet} \\
& \mathrm{R}_{05}: \mathrm{LOO}^{\bullet}+\mathrm{LH} \stackrel{\mathrm{k}_{\mathrm{pi}}}{\longrightarrow} \mathrm{LOOH}+\mathrm{L}^{\bullet} \\
& \mathrm{R}_{06}: \mathrm{LOO}^{\bullet}+\mathrm{LOO}^{\bullet} \stackrel{\mathrm{k}_{\mathrm{tl}}}{\longrightarrow} \text { Termination products } \\
& \mathrm{R}_{07}: \mathrm{LOO}^{\bullet}+\mathrm{L}^{\bullet} \stackrel{\mathrm{k}_{\mathrm{t}}}{\longrightarrow} \text { Termination products } \\
& \mathrm{R}_{08}: \mathrm{L}^{\bullet}+\mathrm{L}^{\bullet} \stackrel{\mathrm{k}_{\mathrm{t}}}{\longrightarrow} \text { Termination products }
\end{aligned}
$$

145 Eq. (1) is realistic, but it remains a simplified description of the full thermo-oxidation

146 mechanism. It involves chemical functions and does not consider their carriers: either TAG or

147 attached FE. Besides, the notation LH is ambiguous as it represents a generic unsaturated FE

148 with several labile hydrogens, whose number and type depend on the considered FE. Labile

149 hydrogens are in $\alpha$ position of a double bond (hold by the first carbon after the double bond).

150 They are not equivalent as their abstraction energies vary from $313 \mathrm{~kJ} \cdot \mathrm{mol}^{-1}$ down to 209

$151 \mathrm{~kJ} \cdot \mathrm{mol}^{-1}$ for mono- (i.e., adjacent to one single unsaturated carbon atom) and bi-allylic (i.e., 152 adjacent to two unsaturated carbon atoms) hydrogen respectively [42]. The relative oxidability 153 of C18:0 (stearate, no unsaturation), C18:1, C18:2 and C18:3 is ranked 1:11:114:179 [43].

154 Combining Eq. (1) and transport during deep-frying can be performed either by introducing a 155 mass balance on the different types of labile protons and subsequent products or by introducing 156 a mass balance on the different chemical functions held by the different unsaturated FE. Both 157 balances are equivalent when the formation of di-, tri-, and cyclic hydroperoxides is discarded. 158 This assumption was considered acceptable in the normal conditions of frying. Discarding FE 
oxidized twice or three times facilitated the reconstruction of the profile of hydroperoxides from

160 real experiments and the design of a thermal activation model.

161 Figure 1 summarizes the multiscale problem with the following key steps: the oil anisothermal

162 flow, oxygen dissolution, transport of reactive species, and the desorption of scission products.

163 At the scale of the oil tank, natural convection regularly exposes the oil to atmospheric

164 dioxygen, where it is rapidly converted into hydroperoxides. Descending plumes remove

165 hydroperoxides from the surface and spread them in the entire volume. As a result, a sharp

166 oxygen density gradient is expected immediately below the oil-air surface. The detailed

167 mechanism of how organic oxygen is transported and reacts within the oil volume remains

168 unexplored, but with experimental arguments showing the speciation of termination reactions

169 between the tank's top and bottom [10]. Coupling Eq. (1) with transport and temperature

170 activation requires reasonable assumptions on carriers. Two stereospecific indices are needed

171 to maintain the separability of carriers LH associated with labile hydrogens: a first one to

172 designate glycerol derivatives' configuration (carbons 1-3 on the glycerol backbone) and

173 another one to indicate the position of the dioxygen addition. Since the combinatory increases

174 rapidly in real lipid mixtures, three approximation levels are suggested in Figure 1c. The

175 coarsest description considers only oxygenated chemical species (hydroperoxide, alcohol, and

176 carbonyl compounds). This choice prevails in the literature and neglects each FE or carrier

177 site's specific reactivity. "Averaged" temperature activation models prevent reaction rates from

178 being transposed from one deep-fryer to another or from oil to another. FE composition effects

179 are recovered by considering either the oil as a mixture of FE or TAG. Replacing one single

180 TAG by three independent FE (regardless of their branching on the glycerol backbone) in

181 numerical models offers the best tradeoff between extrapolation capabilities and complexity.

182 This level coarse-graining process was chosen in this study. 


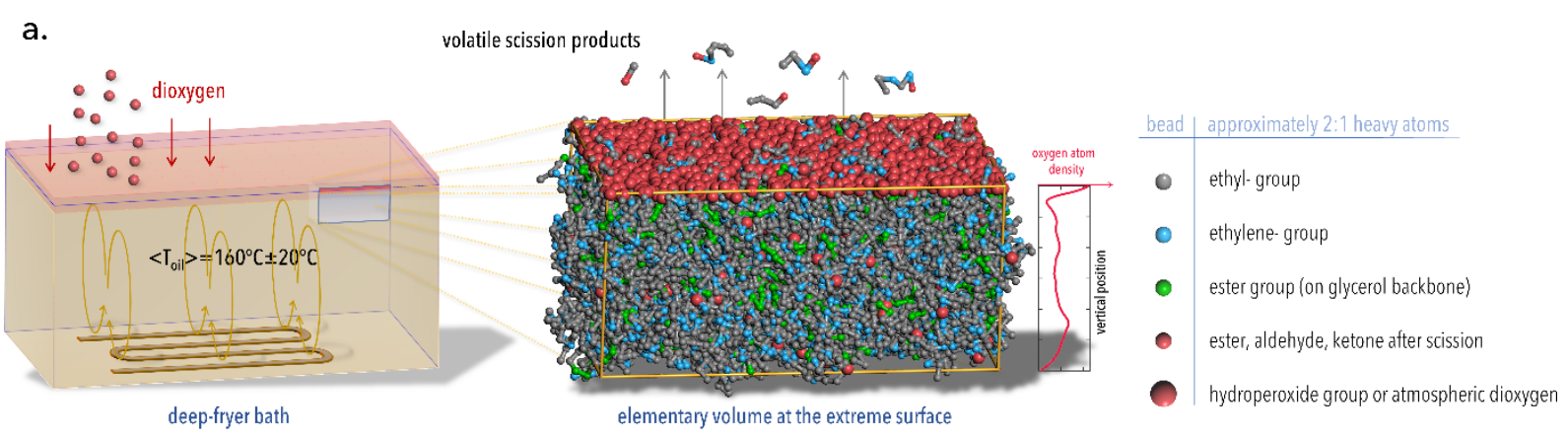

b.

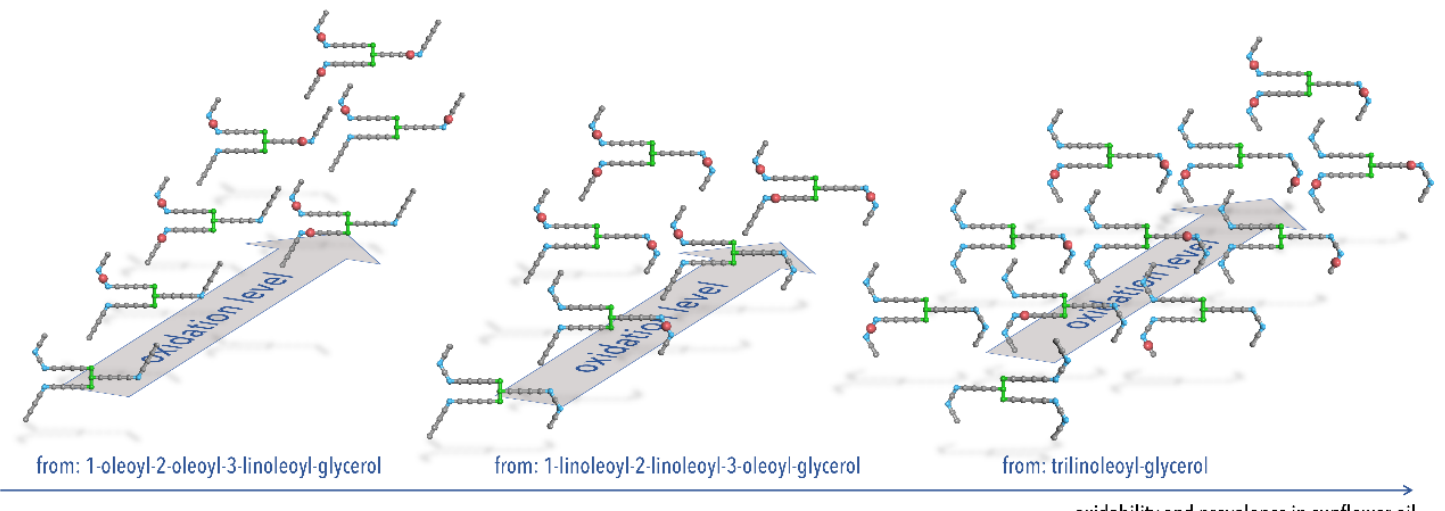

c.

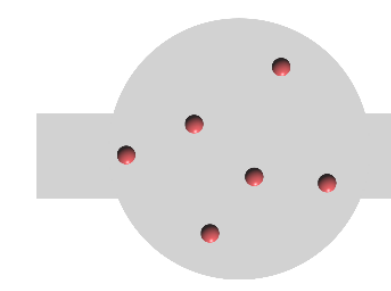

balance in hydroperoxide chemical functions or equivalent $\mathrm{O}_{2}$ :

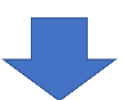

- balance of labile hydrogens

- average reactivity

- no specific thermal activation

- no effect of TAG composition

- no ability to predict the profile of oxidation products

Figure 1: Coarse-grained description of the oxidation process at the scale of the deep-fryer. (a) a coarse-grained molecular description is proposed with building blocks containing two to three heavy atoms. The plotted oxygen profile corresponds to the vertical density of oxygen atoms as calculated on the elementary volume. (b) Illustration of oxygen addition subsequently to a random abstraction of labile hydrogens (i.e., on allylic sites). (c) Pro and cons associated with the loss of molecular details.

\section{Coding the lineage of hydroperoxides}

191 Hydroperoxides of FE, denoted $\mathrm{LOOH}$, interact with native FE (i.e., LH) in a statistical 
oxidation products. The tracking of oxidation products is carried out by maintaining the original

194 FE lineage and introducing prevalence assumptions. For the sake of simplicity, only unsaturated

195 FEs were considered. Minor saturated FEs (laurate, palmitate, and stearate) were discarded

196 because the abstraction energy of aliphatic hydrogens is much higher $\left(418 \mathrm{~kJ} \cdot \mathrm{mol}^{-1}[42]\right)$ than

197 the allylic ones.

198 In this study, FE hydroperoxides were identified with triple indices, as $\mathrm{L}_{\mathrm{i}, \mathrm{j}, \mathrm{k}} \mathrm{OOH}$. All possible

199 hydrogen abstraction cases and subsequent possible rearrangements from C18:1, C18:2, and

$200 \mathrm{C} 18: 3$ are shown in Table 1. The first index $i=1,2,3$ identifies the FE carrier by its number of 201 unsaturations (e.g., $i=2$ codes for C18:2). For the same FE carrier, possible hydroperoxides 202 differ from the position of the hydroperoxide function $(\mathrm{OOH})$ and the configuration of the 203 substituent groups (cis or trans, see [44-46]). $j$ is the isomer index in the delta nomenclature 204 system [47] and $k$ the stereoisomer index. In shorts, 8, 4, and 4 different hydroperoxides can 205 be produced from $i=1,2,3$ respectively. It is worth noticing that the proposed coarse-graining 206 reduced the complexity dramatically to consider. In the real-life, each TAG carries 0 to 3 207 hydroperoxide functions, evolving to one of the $((8+1)+2 \times(4+1))^{3}-((8+1)+2 \times(4+1))^{2}=6498 \quad$ non-palindromic $\quad$ sequences $\quad$ of hydroperoxides. Each of them may exhibit potentially different stabilities with temperature. 
Table 1: Structure of initial fatty esters and hydroperoxides produced by autoxidation and proportion of each isomers at a specific temperature. Empty and filled arrows indicated the position of mono-allylic and bi-allylic hydrogens, respectively.

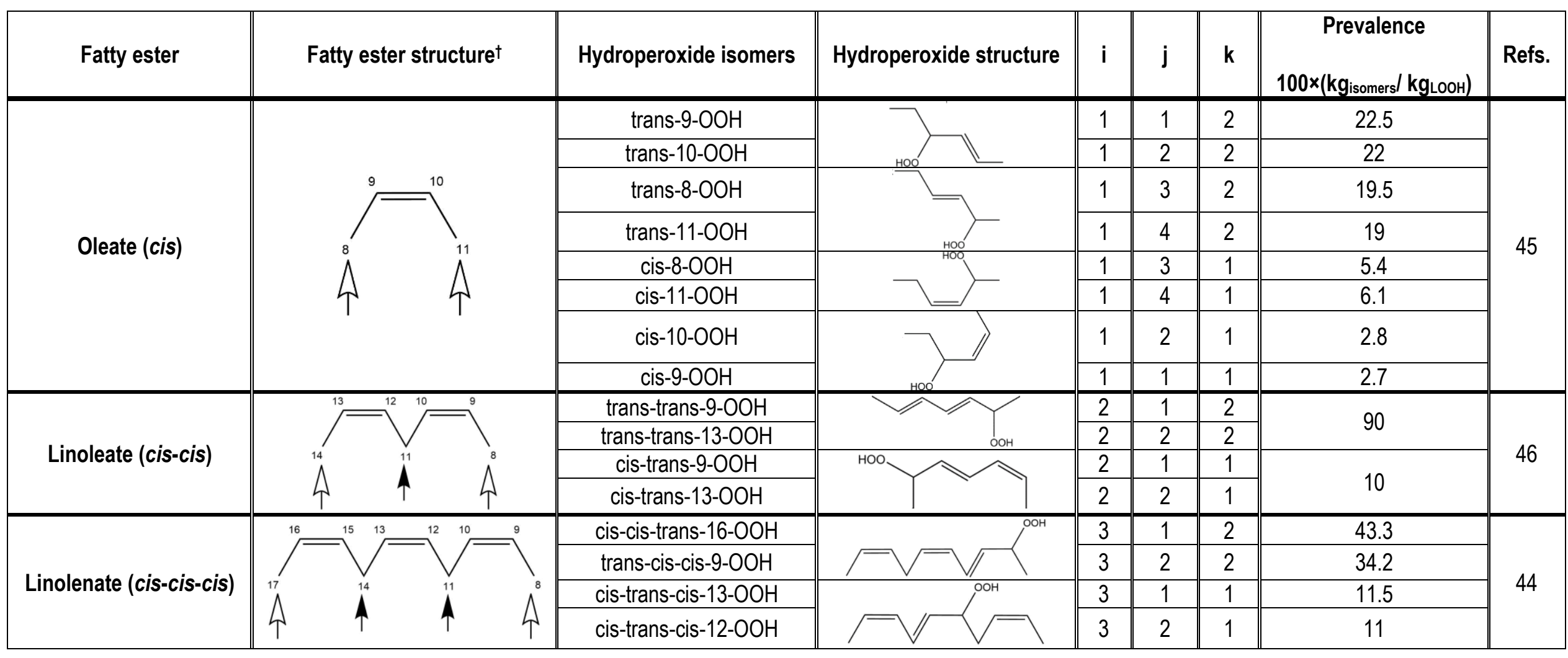

216 [45]: autoxidation at $95^{\circ} \mathrm{C},[46]$ : autoxidation at $75^{\circ} \mathrm{C},[44]$ : autoxidation at $80^{\circ} \mathrm{C}$. tRoman numbers represent the carbon numbers according to the delta nomemclature system 217 [47] 


$$
\left.\left\{i_{1}, j_{1}, k_{1}\right\},\left\{i_{2}, j_{2}, k_{2}\right\},\left\{i_{3}, j_{3}, k_{3}\right\},\left\{i_{4}, j_{4}, k_{4}\right\}\right):
$$

$$
\begin{aligned}
& \mathrm{R}_{01}: \mathrm{L}_{\mathrm{i}, \mathrm{j}, \mathrm{k}} \mathrm{OOH} \stackrel{\mathrm{k}_{\mathrm{i}, \mathrm{j}, \mathrm{k}}}{\longrightarrow} \mathrm{L}_{\mathrm{i}, \mathrm{j}, \mathrm{k}} \mathrm{O}^{\bullet}++^{\bullet} \mathrm{OH} \\
& \mathrm{R}_{02}: \mathrm{L}_{\mathrm{i}, \mathrm{j}, \mathrm{k}} \mathrm{O}^{\bullet}+\mathrm{L}_{\mathrm{i}_{1}, \mathrm{j}_{1}, \mathrm{k}_{1}} \mathrm{H} \stackrel{\mathrm{k}_{\mathrm{b}}}{\longrightarrow} \mathrm{L}_{\mathrm{i}, \mathrm{j}, \mathrm{k}} \mathrm{OH}+\mathrm{L}_{\mathrm{i}_{1}, \mathrm{j}_{1}, \mathrm{k}_{1}} \\
& \mathrm{R}_{03}: \mathrm{HO}^{\bullet}+\mathrm{L}_{\mathrm{i}_{2}, \mathrm{j}_{2}, \mathrm{k}_{2}} \mathrm{H} \stackrel{\mathrm{k}_{\mathrm{b}}{ }^{\prime} \longrightarrow}{\longrightarrow} \mathrm{H}_{2} \mathrm{O}+\mathrm{L}_{\mathrm{i}_{2}, \mathrm{j}_{2}, \mathrm{k}_{2}} \\
& \mathrm{R}_{04}: \mathrm{L}_{\mathrm{i}, \mathrm{j}, \mathrm{k}} \cdot{ }^{\bullet} \mathrm{OO}^{\bullet} \stackrel{\mathrm{k}_{\mathrm{o}}}{\longrightarrow} \mathrm{L}_{\mathrm{i}, \mathrm{j}, \mathrm{k}} \mathrm{OO}^{\bullet}
\end{aligned}
$$

$$
\mathrm{R}_{05}: \mathrm{L}_{\mathrm{i}, \mathrm{j}, \mathrm{k}} \mathrm{OO}^{\bullet}+\mathrm{L}_{\mathrm{i}_{3}, \mathrm{j}_{3}, \mathrm{k}_{3}} \mathrm{H} \stackrel{\mathrm{k}_{\mathrm{p}_{3}, j_{3}, \mathrm{k}_{3}}}{\longrightarrow} \mathrm{L}_{\mathrm{i}, \mathrm{j}, \mathrm{k}} \mathrm{OOH}+\mathrm{L}_{\mathrm{i}_{3}, \mathrm{j}_{3}, \mathrm{k}_{3}} \cdot
$$

$\mathrm{R}_{06}: \mathrm{L}_{\mathrm{i}, \mathrm{j}, \mathrm{k}} \mathrm{OO}^{\bullet}+\mathrm{L}_{\mathrm{i}, \mathrm{j}, \mathrm{k}} \mathrm{OO}^{\bullet} \stackrel{\mathrm{k}_{\mathrm{t1}}}{\longrightarrow}$ Termination products

$\mathrm{R}_{07}: \mathrm{L}_{\mathrm{i}, \mathrm{j}, \mathrm{k}} \mathrm{OO}^{\bullet}+\mathrm{L}_{\mathrm{i}, \mathrm{j}, \mathrm{k}} \stackrel{\mathrm{k}_{\mathrm{1} 2}}{\longrightarrow}$ Termination products

$$
\mathrm{R}_{08}: \mathrm{L}_{\mathrm{i}, \mathrm{j}, \mathrm{k}} \cdot+\mathrm{L}_{\mathrm{i}, \mathrm{j}, \mathrm{k}} \cdot \stackrel{\mathrm{k}_{13}}{\longrightarrow} \text { Termination products }
$$

B. $3 D$ unsteady CFD simulations of turbulent natural convection in a batch deepfryer

\section{Governing equations}

The buoyancy-driven oil flow in the deep-fryer was simulated under the Boussinesq approximation by assuming a constant isobaric thermal expansion coefficient:

$$
\beta=-\frac{1}{\rho}\left(\frac{\partial \rho}{\partial T}\right)_{P}
$$


where $\rho$ is the oil density and $T$ is the oil temperature.

236 The equations of continuity, momentum, and energy are:

237

$$
\begin{aligned}
& \nabla \cdot \mathbf{v}=0 \\
& \rho\left[\frac{\partial \mathbf{v}}{\partial t}+(\mathbf{v} \cdot \nabla) \mathbf{v}\right]=\nabla \cdot(\mu \nabla \mathbf{v})-\nabla p+\rho\left[1-\beta\left(T-T_{0}\right)\right] \mathbf{g} \\
& \rho C_{p} \frac{d T}{d t}=\nabla \cdot(k \nabla T)
\end{aligned}
$$

238 where $\mathbf{v}, p$ and $T$ are the velocity, pressure, and temperature fields, respectively. The

239 dynamic viscosity, $\mu$, the heat capacity, $C_{p}$, and the thermal conductivity, $\lambda$, were allowed to vary with temperature as tabulated in Table $1 . \mathbf{g}$ is the gravitational acceleration.

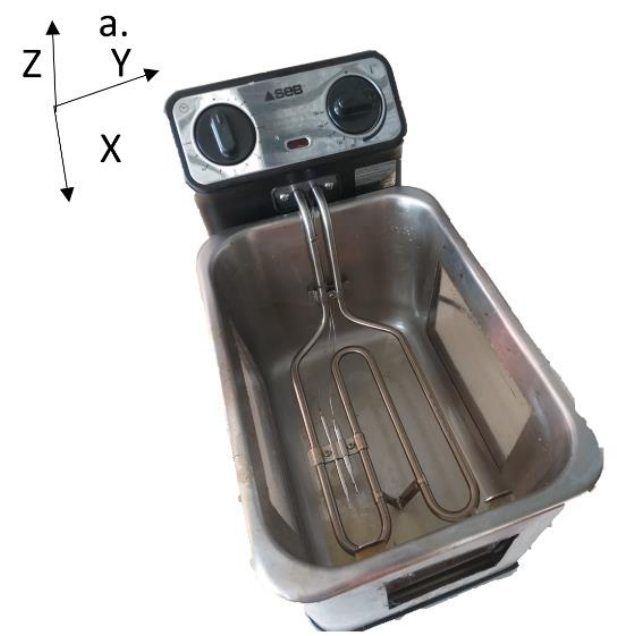

b.
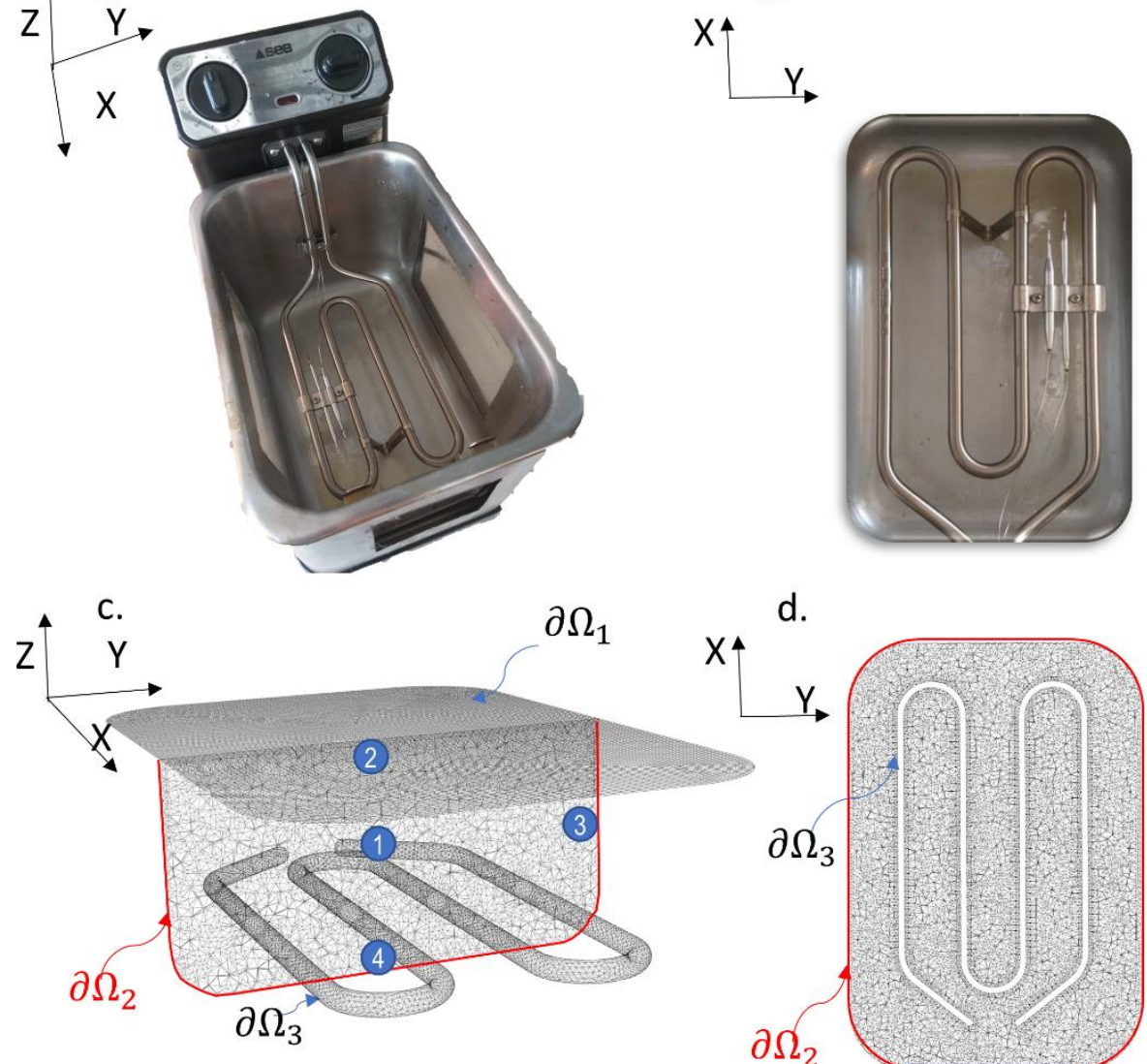

d.

Figure 2. Design of the reference deep-fryer used in this study: (a) overview of the deep-fryer equipped with three windows to image the flow, (b) top view of the tank, (c-d) overview of the mesh. Positions indicated by $1,2,3$ and 4 in blue circles correspond to the center $(X=0, Y=0, Z=0)$, the $\operatorname{top}(X=0, Y=0, Z=0.0514 \mathrm{~m})$, the lateral $(X=0.08, Y=0, Z=0)$ and the bottom $(X=0, Y=0, Z=-0.041 \mathrm{~m})$ respectively. 
No-slip was set for the flow at all boundaries:

$$
\mathbf{v}=0
$$

248 At the walls of the tank $\left(1.2 \mathrm{~mm}\right.$ thick stainless steel for the vertical walls) $\partial \Omega_{2}$ and in 249 agreement with temperature and flux measurements, an effective heat loss by conduction, 250 convection, and radiation (linearized temperature dependence) was applied:

$$
-\left.\mathbf{n}\right|_{\partial \Omega_{2}} \cdot \lambda \nabla T=h_{2}\left(T-T_{\infty}\right)
$$

252

253

At the free surface with air $\left(\partial \Omega_{1}\right)$, a slip boundary condition was applied. Erasing the vertical component of velocity is an acceptable approximation, but, in the real-life, heating plumes at the vertical of the heating elements make the surface rough and turbulent even in the absence of food products. These effects increase the oil surface area exposed to air, and therefore oxygen. A heat loss by convection was enforced at the free surface. Eqs. (6) and (8) assuming that the surrounding temperature, $T_{\infty}$, is kept at $25^{\circ} \mathrm{C}$.

$$
\begin{aligned}
-\left.\mathbf{n}\right|_{\partial \Omega_{1}} \cdot \mathbf{v}= & 0,-\left.\left.\mathbf{t}\right|_{\partial \Omega_{1}} \cdot[-\nabla p+\nabla \cdot(\mu \nabla \mathbf{v})] \mathbf{n}\right|_{\partial \Omega_{1}}=0 \\
& -\left.\mathbf{n}\right|_{\partial \Omega_{1}} \cdot \lambda \nabla T=h_{1}\left(T-T_{\infty}\right)
\end{aligned}
$$

Heat is transferred to oil via a three-coil sheathed tubular heating element (heating surface area: $2.23 \cdot 10^{-2} \mathrm{~m}^{2}$ ) placed above the tank's bottom. Two bulb and capillary thermostats placed in the middle of the first coil control temperature, as shown in Figure 2a. The longest and thinnest bulb positioned near the heating element's bottom plane prevents oil overheating above 200$210^{\circ} \mathrm{C}$. The second bulb is positioned at the mid-height of the heating element and controls the frying temperature. Although very basic, the second sensor's mechanical thermostat offers a somewhat frequency proportional control close to the set temperature. The heating periods are 
long, far from the setpoint, and shorter otherwise. A similar on-off control was implemented

268 numerically by imposing the heat flux, $\boldsymbol{\Phi}_{3}$, at the external wall of the tubular heating element

269 (effective heat flux density $\phi_{3}=9.2 \cdot 10^{4} \mathrm{~W} \cdot \mathrm{m}^{-2}$ ). The skin temperature of the heating element,

$270 T_{\text {skin }}$, was given by:

271

$$
-\left.\mathbf{n}\right|_{\partial \Omega_{3}} \cdot \boldsymbol{\Phi}_{3}^{(t)}=h_{3}\left(T_{s k i n}-T\right)
$$

with $\Phi_{3}=0$ when $T_{\text {frying }}>T_{\text {set }}, \Phi_{3}=\phi_{3}$ otherwise

272 The frying temperature $T_{\text {frying }}$ was defined by frying temperature at position 1 (see Figure 2

$273 \mathrm{X}=0, \mathrm{Y}=0, \mathrm{Z}=0$ ). The set temperature $T_{\text {set }}$ was chosen equal to $165^{\circ} \mathrm{C}$. In the real and simulated

274 oil baths, the skin temperature reached values up to $40^{\circ} \mathrm{C}$ above $T_{\text {frying }}$. All simulations started

275 with an initial temperature of $25^{\circ} \mathrm{C}$ equal to the ambient one $T_{\infty}$.

Table 2. List of parameters used to simulate the anisothermal heat flow within the deep-fryer.

\begin{tabular}{|c|c|c|c|c|}
\hline Parameters & Symbols & Value & Units & Refs. \\
\hline \multicolumn{5}{|c|}{ sunflower oil } \\
\hline density & $\rho$ & $\rho(T)=-0.64 T+1101.1$ with $T$ in $K$ & $\mathrm{~kg} \cdot \mathrm{m}^{-3}$ & [50] \\
\hline $\begin{array}{l}\text { thermal } \\
\text { expansion } \\
\text { coefficient }\end{array}$ & $\beta$ & $7.0 \cdot 10^{-4}$ & $K^{-1}$ & - \\
\hline $\begin{array}{l}\text { dynamic } \\
\text { viscosity }\end{array}$ & $\mu$ & $\begin{array}{c}\mu(T)=4.37 \cdot 10^{-6} \exp \left(-\frac{23.4 \cdot 10^{-6}}{R T}\right) \\
\text { with } T \text { in } \mathrm{K}\end{array}$ & $P a \cdot s$ & [51] \\
\hline $\begin{array}{l}\text { specific heat } \\
\text { capacity }\end{array}$ & $C_{p}$ & $\begin{array}{c}C_{p}(T)=3.477 \cdot 10^{-3}(T-273.15)+2.566 \\
\text { with } T \text { in K }\end{array}$ & $k J \cdot \mathrm{kg}^{-1} \cdot K^{-1}$ & [51] \\
\hline $\begin{array}{l}\text { thermal } \\
\text { conductivity }\end{array}$ & $k$ & $\begin{array}{c}k(T)=0.149+10^{-4} T \\
\text { with } T \text { in } K\end{array}$ & $W \cdot m^{-1} \cdot K^{-1}$ & [52] \\
\hline \multicolumn{5}{|c|}{ deep-fryer (technical specifications) } \\
\hline oil tank length & $L_{e}$ & $26.51 \cdot 10^{-2}$ & $\mathrm{~m}$ & - \\
\hline oil tank width & $W$ & $17.82 \cdot 10^{-2}$ & $\mathrm{~m}$ & - \\
\hline oil height & $H$ & $9.14 \cdot 10^{-2}$ & $\mathrm{~m}$ & - \\
\hline oil volume & $V_{\text {oil }}$ & 4 & $\mathrm{~L}$ & - \\
\hline $\begin{array}{l}\text { oil initial } \\
\text { temperature }\end{array}$ & $T_{0}$ & 25 & ${ }^{\circ} \mathrm{C}$ & - \\
\hline
\end{tabular}




\begin{tabular}{|l|c|c|c|c|}
\hline $\begin{array}{l}\text { oil set } \\
\text { temperature }\end{array}$ & $T_{\text {frying }}$ & 165 & ${ }^{\circ} \mathrm{C}$ & - \\
\hline $\begin{array}{l}\text { maximum } \\
\text { power of the } \\
\text { heating element }\end{array}$ & $P_{\max }$ & 2000 & $\mathrm{~W}$ & - \\
\hline
\end{tabular}

convective heat transfer coefficients (validated experimentally at steady state, $>9$ min after initial heating)

\begin{tabular}{|l|c|c|c|c|}
\hline free surface & $h_{1}$ & $\begin{array}{c}8 \text { from the correlation } N u_{L}=0.14 R a_{L}^{1 / 3} \\
\text { with } L=W\end{array}$ & $W \cdot m^{-2} K^{-1}$ & [53] \\
\hline walls & $h_{2}$ & 2 (experimental determination) & $W \cdot m^{-2} K^{-1}$ & - \\
\hline heating element & $h_{3}$ & $\begin{array}{c}\text { from the correlation } N u_{L}=0.145 R a_{L}^{0.29} \text { with } \\
L=0.07 \mathrm{~m} \text { (oil height above the heating } \\
\text { element) }\end{array}$ & $W \cdot m^{-2} K^{-1}$ & {$[54,55]$} \\
\hline
\end{tabular}

277 where $N u_{L}=\frac{h_{i} L}{\lambda}$ for $i=1,3, R a=\frac{g \beta \Delta T L^{3}}{(\mu / r h o)^{2}} \operatorname{Pr}$ and $\operatorname{Pr}=\frac{\mu C_{p}}{k}$ are Nusselt, Rayleigh and

278 Prandtl dimensionless numbers, respectively.

\section{Numerical resolution}

281 Eq. (3)-(9) were resolved in time and space numerically with the CFD software Ansys-Fluent 282 (version 17, Ansys, USA). Velocity and temperature fields inside the tank were simulated 283 during the first 10-12 minutes of heating until an apparent steady-state was observed in the 284 region above the heating element. Ansys-Gambit (version 2.4.6) has been used for mesh 285 generation and applied to deep-fryer sketched in 3D with an accuracy of $\pm 0.2 \mathrm{~mm}$. The mesh 286 validation study showed an optimal mesh consisting of 637186 tetrahedral cells for the entire 287 oil bath, with the finest mesh size chosen as $\delta_{\min }=\min \left(\frac{L}{2 N u_{L}}\right) \sim 1.5 \mathrm{~mm}$ [55] to capture thermal 288 and dynamic boundary layer effects near cold and hot surfaces. The Nusselt number imposed 289 the minimal thickness at the heating elements (where $L$ is the external diameter of the heating 290 element).

291 The second-order SIMPLE scheme (Semi Implicit Method for Pressure Linked Equations) was 292 used to integrate the pressure-velocity equations in time. Simulations were run up to $2 \times 10^{4}$ time 
293 steps with a fixed time step size chosen as $\Delta t=\frac{\Delta x_{\min }}{v_{\max }}=0.0542$ s. $\Delta x_{\min }=2 \mathrm{~mm}$ was chosen

294 slightly larger than the finest cell's size at the vicinity of the heating element. The maximum 295 velocity was estimated conservatively as $v_{\max }=1.05 \frac{\lambda}{\rho L C_{p}} \operatorname{Ra}_{L}^{0.43} \operatorname{Pr}^{1 / 3}$ by fitting the maximum 296 turbulent velocity determined experimentally in a Rayleigh-Bénard water tank for an equivalent $297 R a \sim 10^{7}$ [55]. It was previously verified on this equivalent configuration that the turbulence 298 treatment would not require Direct Numerical Simulation (DNS). LES (Large-Eddy 299 Simulation) was, therefore, applied for this study. Unresolved small-scale details, particularly 300 the transported temperature-field near boundaries, were estimated with the Smagorinsky-Lilly 301 subgrid-scale model acting as a low-pass filter.

\section{Hydroperoxide decomposition model along streamlines}

303 Figure 1 illustrates the likely coupling between the transport of chemical species after exposure 304 to atmospheric oxygen in regions with different temperatures. This study focuses on the thermal decomposition of $L_{i, j, k} O O H$ considering that they are produced from the top surface, and they are subsequently dispersed by the flow in the bulk volume, including beneath the heating element. The residence time of hydroperoxides away from the surface limits the effective 308 thermo-oxidation rate when the propagation rate is close to the decomposition rate of $L_{i, j, k} O O H$ (i.e., reaction chain length close to unity). This condition is identified on the

310 oxidation kinetics by a concentration maximum in total hydroperoxides (i.e., they are renewed 311 as fast they are decomposed. A detailed map of residence times was required to evaluate the

312 lifetimes of $L_{i, j, k} \mathrm{OOH}$ in the presence of large oil temperature heterogeneities (i.e., up to $80^{\circ} \mathrm{C}$ ).

313 Eulerian descriptions of decomposition kinetics were not favored as they do not offer direct 314 relationships between residence times and recirculation loops. Comparatively, Lagrangian 
315 descriptions can deal efficiently with any source (point, surface, and volume) and are

316 straightforward to implement for unimolecular reactions (first-order).

317 The variation of hydroperoxide concentration of a fluid particle $p$ along the streamline $\aleph_{p}$

318 equipped with the curvilinear coordinate $\ell$ corresponding to the cartesian coordinates $(x, y, z)$

319 depends on the local residence time $\left.\left.\mathbf{U}\right|_{x, y, z} \cdot \boldsymbol{\theta}\right|_{\ell} ^{\aleph_{i}}$ and the local temperature $\left.T\right|_{x, y, z}$. The

320 unimolecular hydroperoxide decomposition along $\aleph_{p}$ reads:

321

$$
\begin{aligned}
\left.\frac{d\left[\mathrm{~L}_{\mathrm{i}, \mathrm{j}, \mathrm{k}} \mathrm{OOH}\right]}{d \ell}\right|_{\ell} ^{\aleph_{p}} & =\left.\left.\frac{d\left[\mathrm{~L}_{\mathrm{i}, \mathrm{j}, \mathrm{K}} \mathrm{OOH}\right]}{d t}\right|_{\ell} ^{\aleph_{p}} \frac{d t}{d \ell}\right|_{\ell} ^{\aleph_{p}} \\
& =-\left.k_{d_{i, j, k}}\left(\left.T\right|_{x, y, z}\right)\left[\mathrm{L}_{\mathrm{i}, \mathrm{j}, \mathrm{k}} \mathrm{OOH}\right]\right|_{\ell} ^{\aleph_{p}} \frac{1}{\left.\left.\mathbf{U}\right|_{x, y, z} \cdot \boldsymbol{\theta}\right|_{\ell} ^{\aleph_{p}}}
\end{aligned}
$$

322 where $\left.\mathbf{U}\right|_{x, y, z}$ is the local velocity vector oil and $\left.\boldsymbol{\theta}\right|_{\ell} ^{\mid x_{p}}$ is the unitary vector tangent to the 323 streamline $\aleph_{p}$.

324 In anoxia conditions (i.e., without hydroperoxide regeneration), the local concentration in any 325 location of the deep-fryer is obtained by averaging the decomposition over a representative set 326 of trajectories starting from a reference region (e.g., surface) and joined in the same point $327(x, y, z)$. By denoting $\left\{\ell_{x, y, z}^{\aleph_{p}}\right\}_{p=1 . . N}$ the lengths of the $N$ trajectories in the set, the variation of 328 hydroperoxide content becomes for an arbitrary initial distribution of hydroperoxides:

$$
\left.\left[\mathrm{L}_{\mathrm{i}, \mathrm{j}, \mathrm{k}} \mathrm{OOH}\right]\right|_{x, y, z}=\left\langle\left.\left[\mathrm{L}_{\mathrm{i}, \mathrm{j}, \mathrm{k}} \mathrm{OOH}\right]\right|_{\text {initial }} ^{\aleph_{p}}\right\rangle-\left\langle\left.\int_{0}^{\aleph_{x, y, z}^{\aleph_{p}}} \frac{d\left[\mathrm{~L}_{\mathrm{i}, \mathrm{j}, \mathrm{k}} \mathrm{OOH}\right]}{d \ell}\right|_{l} ^{\aleph_{p}} d l\right\rangle
$$

where $\langle X\rangle$ is the mean of $X$ along $N$ trajectories. 
331 By noticing that $\left.\frac{d \ln \left[\mathrm{L}_{\mathrm{i}, \mathrm{j}, \mathrm{k}} \mathrm{OOH}\right]}{d \ell}\right|_{\ell} ^{\aleph_{p}}=-\frac{k_{d_{i, j, k}}\left(\left.T\right|_{x, y, z}\right)}{\left.\left.\mathbf{U}\right|_{x, y, z} \cdot \boldsymbol{\theta}\right|_{\ell} ^{\aleph_{p}}}$ for first-order kinetics, the variation of 332 hydroperoxide content is also related to the distribution of residence times at different 333 temperatures for considered trajectories:

$$
\begin{aligned}
\ln \left(\frac{\left.\left[\mathrm{L}_{\mathrm{i}, \mathrm{j}, \mathrm{k}} \mathrm{OOH}\right]\right|_{x, y, z}}{\left\langle\left.\left[\mathrm{~L}_{\mathrm{i}, \mathrm{j}, \mathrm{k}} \mathrm{OOH}\right]\right|_{\text {initial }} ^{\aleph_{p}}\right\rangle}\right) & =\left\langle\left.\int_{0} \frac{d \ln \left[\mathrm{L}_{\mathrm{i}, \mathrm{j}, \mathrm{k}} \mathrm{OOH}\right]}{d \ell}\right|_{l} ^{\aleph_{p}} d l\right\rangle=-\left\langle\int_{0}^{\aleph_{x, y, z}^{\aleph_{p}}} \frac{k d_{i, j, k}\left(\left.T\right|_{x, y, z}\right)}{\left.\left.\mathbf{U}\right|_{x, y, z} \cdot \boldsymbol{\theta}\right|_{\ell} ^{\aleph_{p}}} d l\right\rangle \\
& \approx \sum_{q=1 . . N_{T}} k_{d_{i, j, k}}\left(T_{q}\right)\left\langle\tau_{T_{q}}\right\rangle
\end{aligned}
$$

335 where $\left\langle\tau_{T_{q}}\right\rangle$ is the averaged residence time at temperature $T_{q}$ with $q=1 \cdots N_{T} ; N_{T}$ is the number classes uses to discretizes the considered temperature range.

D. Validation of an activation model for the decomposition of $L_{i, j, k} O O H$ in mixtures in isothermal conditions

According to Table 1 and Eq. (2), sixteen different hydroperoxides may be decomposed according to sixteen different rate constants. All individual values cannot be determined experimentally because the different $\mathrm{L}_{\mathrm{i}, \mathrm{j}, \mathrm{k}} \mathrm{OOH}$ cannot be produced independently, even from a pure FE. Reconstructing their concentrations from TAG or FE initial composition is an option but at the expense of adding unknown production or renewal rates.

344 A feasible approach was followed by tracking only the likeliest hydroperoxide isomers of each

345 FE, as supported by qualitative experiments on pure FE. Since hydroperoxides from oleate, 346 linoleate, and linolenate exhibit different thermal stability $[48,56]$, the index $i$ was kept. 347 Among stereoisomers, trans-hydroperoxides, conjugated or not, are more stable and prevail at 348 high temperatures, so that only the case $k=2$ was considered. For instance, $83 \%$ of trans oleate hydroperoxides are present at $95^{\circ} \mathrm{C}$, and $90 \%$ of conjugated trans-trans linoleate 
hydroperoxides are present at $75^{\circ} \mathrm{C}$. Additionally, since isomers $13-\mathrm{OOH}$ and $9-\mathrm{OOH}$

351 hydroperoxides of linoleate have similar lifetimes at high temperatures [57], the proposed mass 352 balance did not separate them ( or $j=2$ ), and the less probable isomer 11-OOH was neglected 353 (see the justification in Ref. [58]). As a result, indices $j$ and $k$ were dropped and $\mathrm{L}_{\mathrm{i}} \mathrm{OOH}$ 354 represented the likeliest mixture of $\mathrm{L}_{\mathrm{i}, \mathrm{j}=1 \text { or } 2, k=2} \mathrm{OOH}$, with $i=1,2,3$.

The rationale for the transposition of constant rates from pure FE to complex TAG mixtures 356 (with $3 \mathrm{FE}$ attached to glycerol) was tested on two oils from 40 to $120^{\circ} \mathrm{C}$ : poppyseed $(13 \%$ oleate, $75 \%$ linoleate, and $0.6 \%$ linolenate, from Ref. [59]) and linseed (22\% oleate, $13 \%$ 358 linoleate and 54\% linolenate, from Ref. [60]). All oxidation kinetics were obtained in isothermal conditions under mechanical mixing. Reported experimental peroxide values, expressed in milli-equivalents peroxide or $\mathrm{O}_{2}$ per $1 \mathrm{~kg}$ of fat (see interpretation Figure 1c), were related to 361 the total amount of hydroperoxides (i.e., regardless of the FE or TAG carrier) as:

$$
[\mathrm{LOOH}]_{\text {total }}^{(t)}=\sum_{i=1}^{3}\left[\mathrm{~L}_{\mathrm{i}} \mathrm{OOH}\right]^{(t)}
$$

363 Since hydroperoxides were obtained during or after oxygen exposure, the corresponding $\left[\mathrm{L}_{\mathrm{i}} \mathrm{OOH}\right]$ kinetics were reconstructed from $[\mathrm{LOOH}]_{\text {total }}^{(t)}$ ones using a simplified version of 365 Eqs. (2), as detailed in Appendix A. Typical reconstructed kinetics during the decomposition366 dominant phase are shown in Figure 3. Aerated and anoxia conditions reproduced those 367 encountered at the surface and in the oil bulk volume without temperature gradient. 368 Decomposition rate constants, $k_{d_{i}}$, were read from Figure 4. Propagation rate constants $k_{p_{1}}$ and $369 k_{p_{2}}$ were fitted from experimental data in the presence of oxygen (no fitting required in anoxia) 370 [61]. Predictions were highly acceptable at $80^{\circ} \mathrm{C}$ and above, i.e. in the frying temperature range. 371 At $40^{\circ} \mathrm{C}$, the time to reach a steady-state (maximum of $[\mathrm{LOOH}]_{\text {total }}^{(t)}$ ) was underestimated, and 
372 the effective decomposition rate was overestimated (see Figure 3e-f). At ambient temperatures,

373 hydroperoxides' stabilization by hydrogen bonds would favor bimolecular decompositions 374 (cage-effect mechanism) instead of unimolecular ones.

375 The preliminary validation in isothermal conditions generalized to mixtures oxidation schemes 376 proposed for thin films or homogeneous conditions (see Ref. [61]). The remainder of the study 377 will extrapolate previous results from isothermal to anisothermal conditions by introducing an 378 activation energy for the decomposition of each $L_{i} O O H$. As the corresponding activation 379 entropies capture the decomposition's molecularity, only reference kinetics above $80^{\circ} \mathrm{C}$ were 380 subsequently considered. 

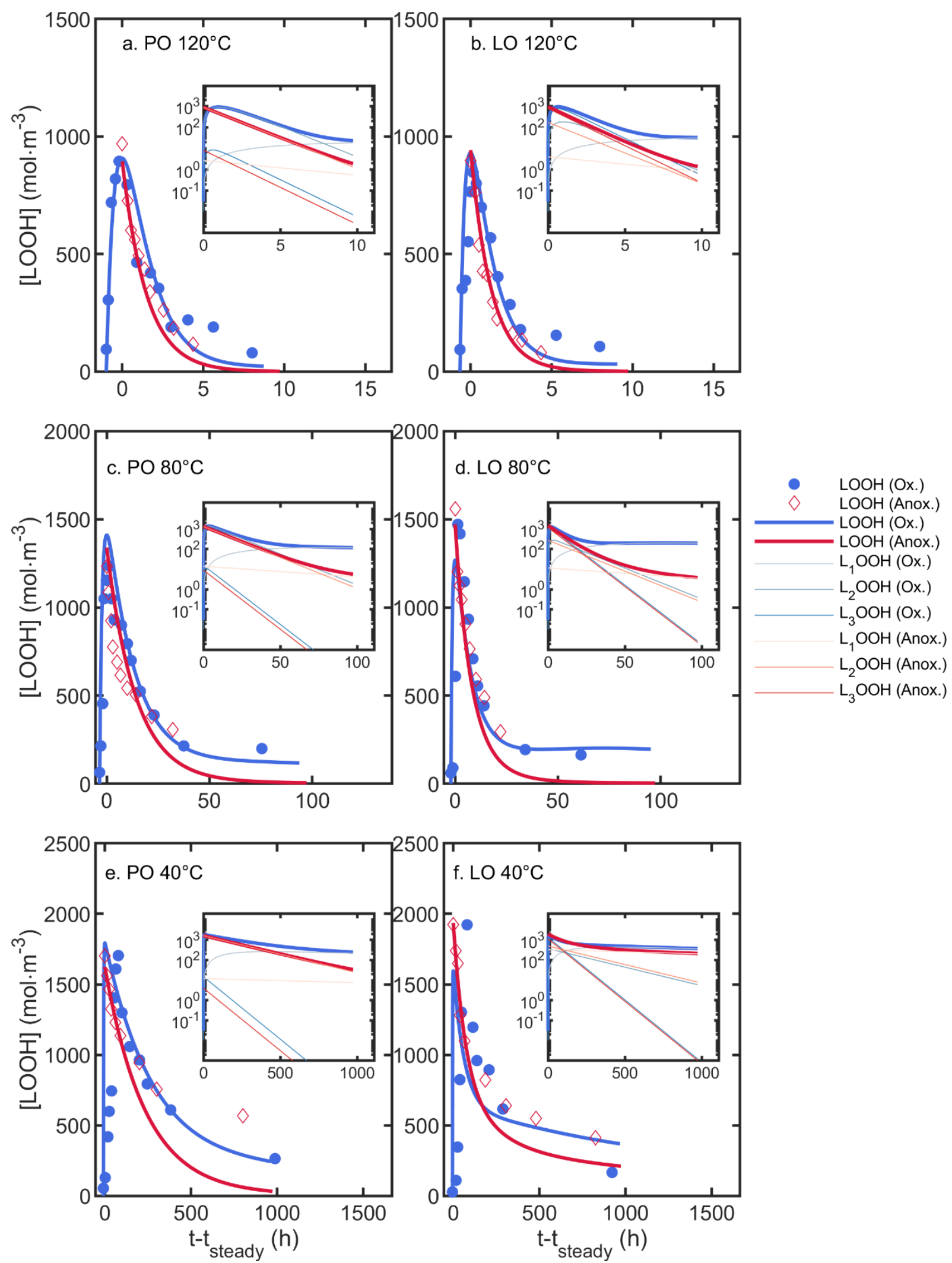

Figure 3: Comparison between predicted (lines) and experimental (symbols) kinetics of total hydroperoxide content in oxygenated conditions (filled symbols) and in anoxia (empty symbols). Experimental data in poppyseed oil (PO) and linseed oil (LO) are from Ref. [61]. The different subpopulations of hydroperoxides are presented in the inlets. When index $i$ has been dropped, the value represents the total concentration in hydroperoxides. 


\section{EXPERIMENTAL METHODS}

A. Oil and fatty acid methyl esters

389 Fresh sunflower oil (SO) was supplied directly by the Lesieur's refinery production plant of

390 Coudekerque (France). Methyl oleate (Alda Aesar, purity of 96\%), methyl linoleate (Acros 391 Organics, purity of 99\%), and methyl linolenate (Sigma Aldrich, purity of 99\%) were 392 purchased.

B. Deep-fryer

394 The reference deep-fryer used in this study was a commercial kitchen appliance (model Semi395 Pro, SEB, France, oil capacity 4 L, 2052W) including an immersed heating element (surface area: $222.7 \mathrm{~cm}^{2}$ ). A second deep-fryer with an alternative design was also considered to highlight the effect of design on the decomposition of hydroperoxides and indirectly on oil 398 lifetime.

\section{Bubbling reactor}

400 Reaction rates of hydroperoxide decomposition of pure FE were determined in a miniaturized 401 bubbling reactor (vertical tube reactor of capacity $30 \mathrm{~mL}$ ), based on the principles as the larger 402 one described in Ref [11]. The FE temperature was controlled by immersing the glass reactor 403 within a large secondary oil bath $(0.5 \mathrm{~L}$, heating power $600 \mathrm{~W})$ equipped with a PID 404 temperature-controller. Dry air or nitrogen was injected from the bottom of the test tube with 405 the help of a Pasteur pipette to reproduce aerated or anoxia conditions, respectively. 
407 The total hydroperoxide content was determined experimentally by differential scanning 408 calorimetry (DSC) according to the method described in Ref. [62]. A small oil aliquot (10-20 $409 \mathrm{mg}$ ) was heated from $25^{\circ} \mathrm{C}$ to $250^{\circ} \mathrm{C}$ at a rate of $3^{\circ} \mathrm{C} / \mathrm{min}$. Since the endothermic decomposition 410 of hydroperoxides in the pan occurs in anoxia, the formed radicals recombine together 411 exothermically into stable products. The total amount of hydroperoxides was quantified by 412 assuming an effective enthalpy of decomposition of $320 \mathrm{~kJ} / \mathrm{mol}$.

\section{E. Temperature measurements}

414 Local temperatures in the oil bath were recorded with thermocouples (type K, TC SA, France) 415 connected to a data acquisition system (Ni cDAQ-9178 and module NI 9213, National 416 Instruments, Austin, Texas, USA) at a frequency of $3 \mathrm{~Hz}$. Temperatures at the surface of the oil 417 bath were imaged with a thermal camera (model Ti9, Fluke, USA) with a emissivity of 0.95.

\section{IV. Results and Discussion}

\section{A. Parametrization of the hydroperoxide decomposition kinetic model}

The assumption of first-order decomposition kinetic of all $L_{i} O O H$ was tested on pure FEs,

421 including seven kinetics of oleate hydroperoxide (ranging from $80^{\circ} \mathrm{C}$ and $180^{\circ} \mathrm{C}$ ), five kinetics 422 of linoleate hydroperoxides (ranging from $120^{\circ} \mathrm{C}$ to $180^{\circ} \mathrm{C}$ ), and seven kinetics of linolenate 423 hydroperoxides (ranging from $100^{\circ} \mathrm{C}$ to $180^{\circ} \mathrm{C}$ ). Decompositions were monitored in the 424 bubbling reactor in strict anoxia. Normalized kinetics are shown as semi-log plots in Figure 4a$425 \mathrm{c}$ with corresponding Arrhenius plots depicted in Figure 4d along with reaction rates reported 426 in the literature. All linearized plots confirmed unimolecular decomposition in frying 427 conditions. Strong activation of decomposition by temperature was evidenced for all 428 hydroperoxides with a lifetime reduction varying from 50 to 400 from $80^{\circ} \mathrm{C}$ to $180^{\circ} \mathrm{C}$. Oleate 

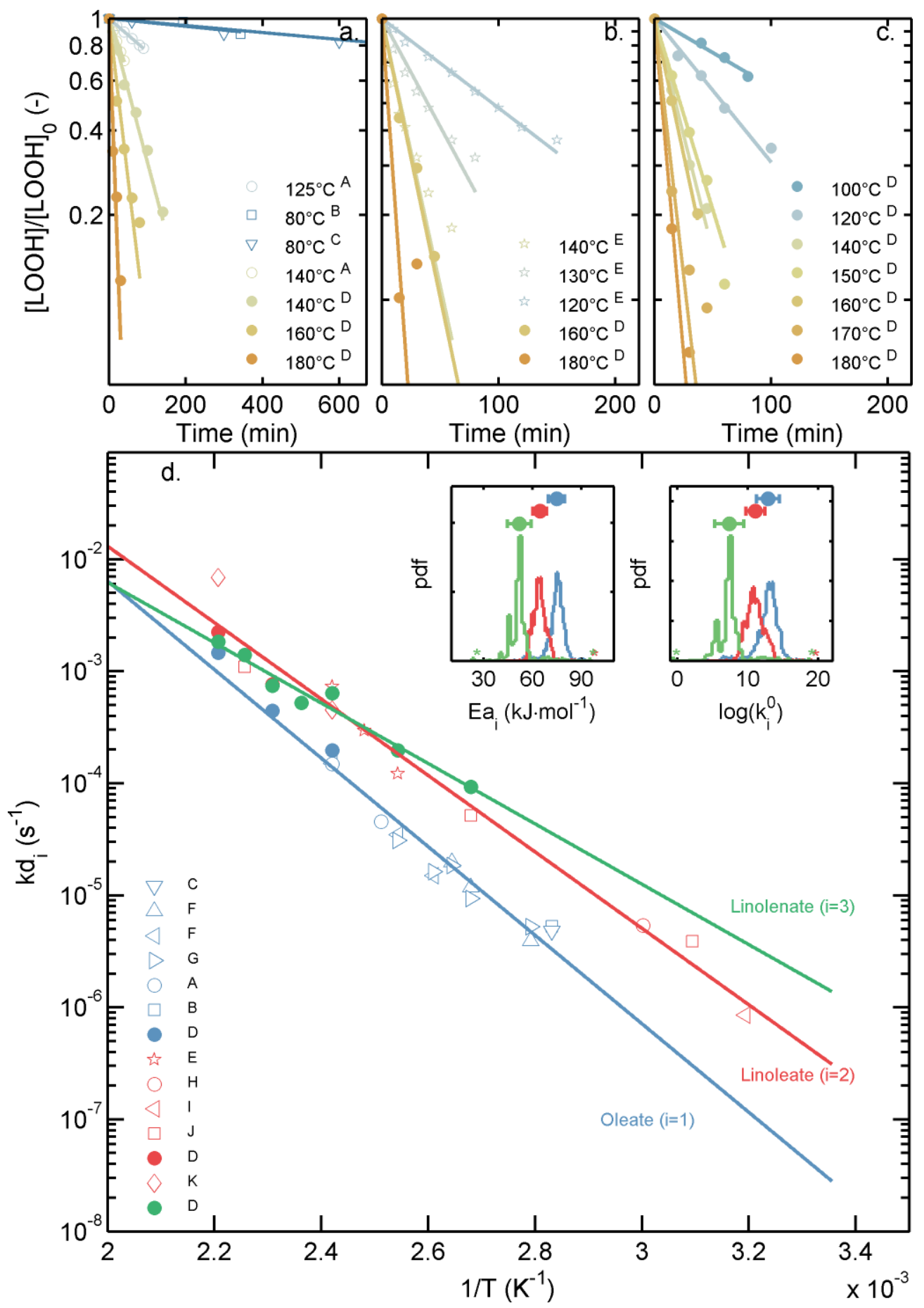

Figure 4. Kinetics of decomposition of hydroperoxides of pure esters (a) methyl oleate (MO), (b) methyl linoleate (ML), (c) methyl linolenate (MLn) in frying conditions, and (d) their corresponding decomposition rate constants and activation energies. Empty symbols are from literature: A from Ref. [38] (ethyl oleate), B from Ref. [39] (MO), C from Ref. [40], E from Ref. [63], F from Ref. [26], G from Ref. [25], H from Ref. [64], I from Ref. [65], J from Ref. [24] and $\mathrm{K}$ from Ref. [66].Filled symbols are from this work and produced in anoxia (nitrogen flow rate of $0.5 \mathrm{~L} \cdot \mathrm{min}^{-1}$ ) in the bubbling reactor. 
439 Decomposition rate constants were fitted with an Arrhenian activation model:

$$
k_{d_{i}}(T)=k_{i}^{0} \exp \left(\frac{-E a_{i}}{R T}\right) \text { with } \mathrm{i}=1,2,3
$$

441 where $k_{i}^{0}$ is the pre-exponential factor, $E a_{i}$ the activation energy, $R$ the gas constant, and $T$ is

442 absolute temperature. The distributions of activation energies and pre-exponential factors due

443 to uncertainties were estimated by bootstrap (1000 samples). It was particularly noticeable that

444 all hydroperoxides' decomposition rates looked similar at $180^{\circ} \mathrm{C}$ whereas they were different 445 at $80^{\circ} \mathrm{C}$. It is, therefore, hinted that all $\mathrm{FE}$ hydroperoxides propagate oxidation at the highest 446 frying temperature, whereas only the most labile ones do during initial heating.

B. CFD results

\section{Validation during the initial heating period}

449 Simulated 3D temperature and velocity fields during the heating step were recorded with a 450 temporal resolution of $\sim 54 \mathrm{~s}$. They are plotted along three principal planes of the deep-fryer in 451 Figure 5. Along the smallest vertical cross-section XZ, the four heating element branches 452 generate four ascensional plumes starting as shown at $\mathrm{t}=54 \mathrm{~s}$. Two convections cells are fully 453 developed after one min and are maintained throughout the whole heating period (up to $542 \mathrm{~s}$ ). 454 The circulation operates mainly within the plane $\mathrm{XZ}$ without a significant mixing in the plane 455 YZ. The ascensional sections are small and associated with ascensional velocities up to 2.8 $456 \mathrm{~cm} \cdot \mathrm{s}^{-1}$. Descent regions are located along the walls are thicker with velocities up to $4 \mathrm{~mm} \cdot \mathrm{s}^{-1}$ 457 The region below the heating element remains stagnant with delayed heating. 


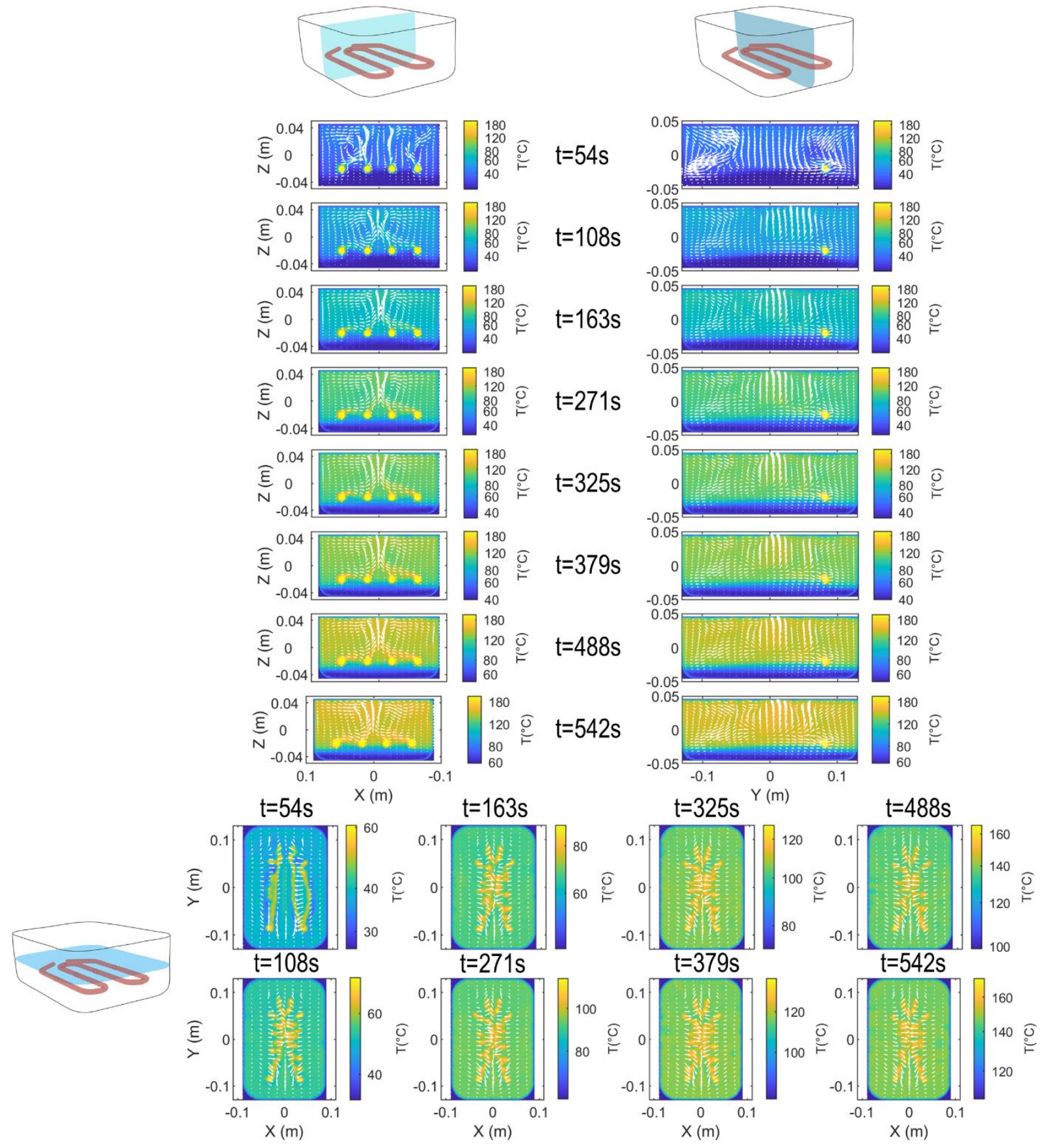

Figure 5: Temperature and velocity distributions during initial heating in three principal planes of the deep fryer

461 Measured temperature kinetics and surface temperatures are shown in Figure 6. Simulated

462 temperatures at the center-of-mass and $20 \mathrm{~mm}$ below the heating element are also given. They

463 were in good agreement. Measured surface temperatures confirmed hot spots aligned with

464 elements coils, but each of them separated by 3 to $4 \mathrm{~cm}$. Surface temperatures were

465 heterogeneous, with variations reaching up to $15-20^{\circ} \mathrm{C}$ while continuous heating was applied. 
Once the set temperature was reached after ca. 9 min, surface heterogeneities decreased down

468 the heating element, but it would have required 2 hours to reach steady-state below the heating element.

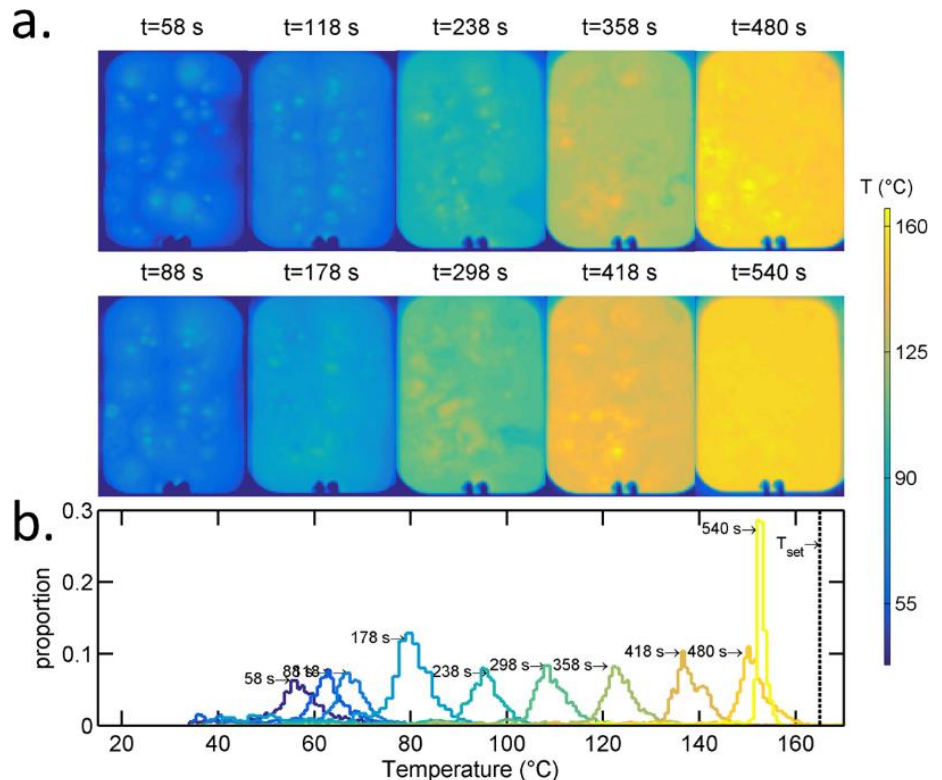

C.

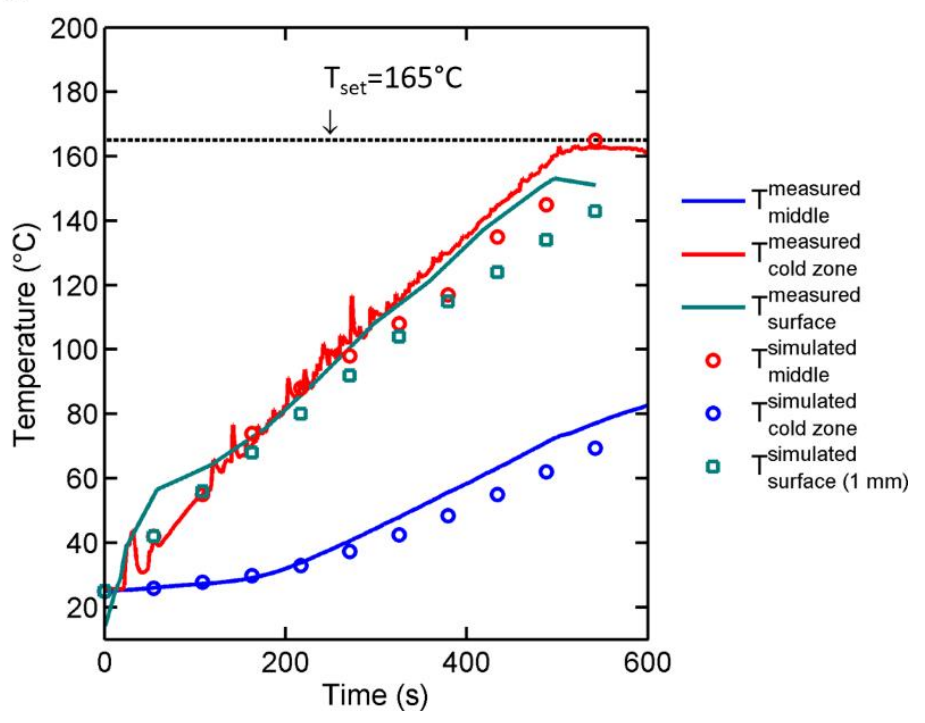

471 Figure 6. a) Thermal imaging of the oil bath surface during the heating period and b) the corresponding temperature 472 distribution and c) the comparison between measured (continuous lines) and simulated (symbols) local temperature. 
474 Simulation's consistency was evaluated by plotting in Figure 7 the temperature $z$-profile along 475 the bath's centreline. Profile details near the free surface (Figure $7 \mathrm{~b}$ ) show a ca. $3 \mathrm{~mm}$ thick 476 boundary layer, that matches the thermal boundary layer simulated from DNS calculations with $477 \quad R a_{L}^{-0.291}[67,68]$.
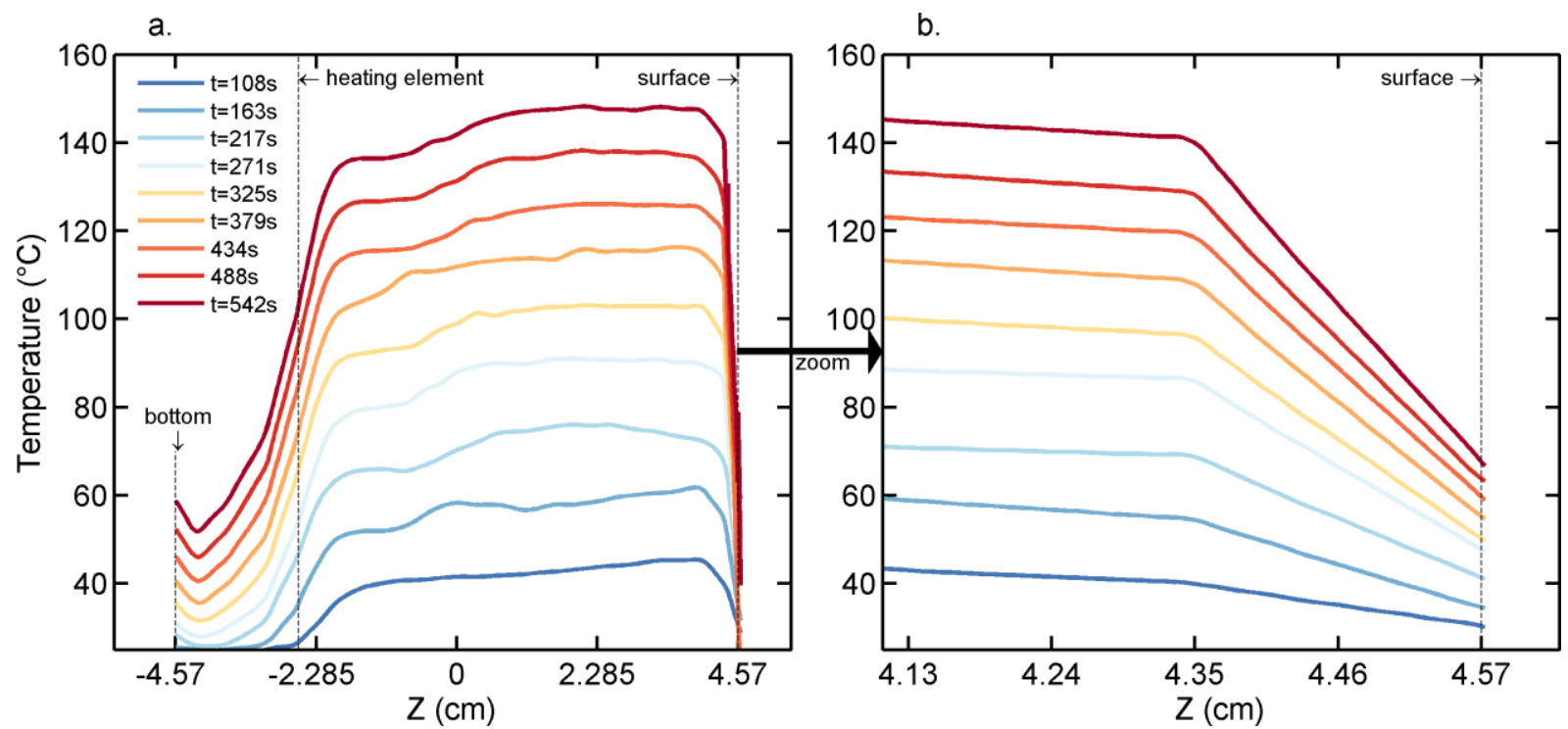

Figure 7: a) Kinetics of temperature profile along $\mathrm{Z}$ during heating and b) zoom near the surface region

Oil flew as smooth plumes at the vertical of the heating coils before spreading in multiple whorls and eddies. Large-scale turbulent patterns were visually revealed by the change of the refraction index and the displacements of solid particles (see, for example, Figure 9b). The flow was asymmetrically structured between ascending and descending flows. Hot plumes rose rapidly in an almost laminar fashion before spreading rapidly along the shortest cross-section. The descent was conversely more turbulent and dissipated progressively in the entire volume. Turbulence on small scales was analyzed in Figure 8 from the temporal fluctuations of velocities and temperatures at four typical positions in the deep-fryer (reported in Figure 2). Although the entire system was out of equilibrium during heating, velocity fluctuations were thought ergodic enough on short and intermediate length scales so that Taylor's hypothesis of 
frozen turbulence held. Normalized spectra of velocity autovariance are plotted vs. natural

493 frequency, $f$ in Figure 8a. Its interpretation within the Kolmogorov turbulence phenomenology

494 requires to relate temporal fluctuations with spatial ones. By assuming that the convected eddy

495 pasts the probe with a mean convection velocity $U$ and unchanged characteristics, the frozen

496 turbulence assumption associates the wavelength $L / n$ with $f=n U / L . L$ represents the

497 largest length scale and $n$ any number greater than 1. All Power spectra were similar and

498 exhibited power-law decays as $f^{-m}$ with exponents $m \approx 5 / 3$ at intermediate frequencies

499 ranging from 0.4 and $8 \mathrm{~Hz}$. Spectra invariant with position and 5/3's scaling signed locally

500 isotropic turbulence in the inertial subrange, where turbulent kinetic energies cascaded from

501 production scales (low frequencies) down to dissipation scales (high frequencies) [69]. This

502 result, never shown in a deep-fryer, suggests an efficient mixing down to the millimeter scale

503 within the deep-fryer due to turbulence. On intermediate time scales, the power spectra of

504 temperature fluctuations decreased rapidly with $f$, but not isotropically or in a self-similar

505 manner. Simulated and measured spectra were quite similar and were approximately associated

506 with an exponent $m \approx 7 / 5$, in agreement with stratified turbulence $[70,71]$ The stratification

507 was exemplified by fluctuations of velocities and temperatures three magnitude orders lower

508 below the heating element (bottom position).

509 The flow described with a deep-fryer under constant heating differed from the classic

510 configuration of Rayleigh-Bénard natural convection cells [68, 72]. In particular, fluctuations

511 did not decrease at the vicinity of the free surface and did not increase at the deep-fryer center.

512 The differences arose from the heating element's geometry covering only a small fraction of

513 the deep-fryer's bottom, the heat loss at the surface. The presence of a cold zone but not

514 isothermal below the heating element also affected the gap between the vertical walls and the

515 heating element. The contribution of heating on temperature fluctuations at the centre of the

516 deep-fryer were analysed by plotting the windowed mean square of experimental fluctuations 
517 at different time scales, $\tau=1 / f$, in Figure $8 \mathrm{c}$. The passage of plumes is visible on short time 518 scales with an external envelope decreasing exponentially according to two times scales: $28 \mathrm{~s}$ 519 and $127 \mathrm{~s}$. The fastest decay was associated with the oscillations of plumes and the longest to 520 vertical mixing. Fluctuations were lower than $\pm 2.1^{\circ} \mathrm{C}$ at steady state and were independent $\tau$ 521 

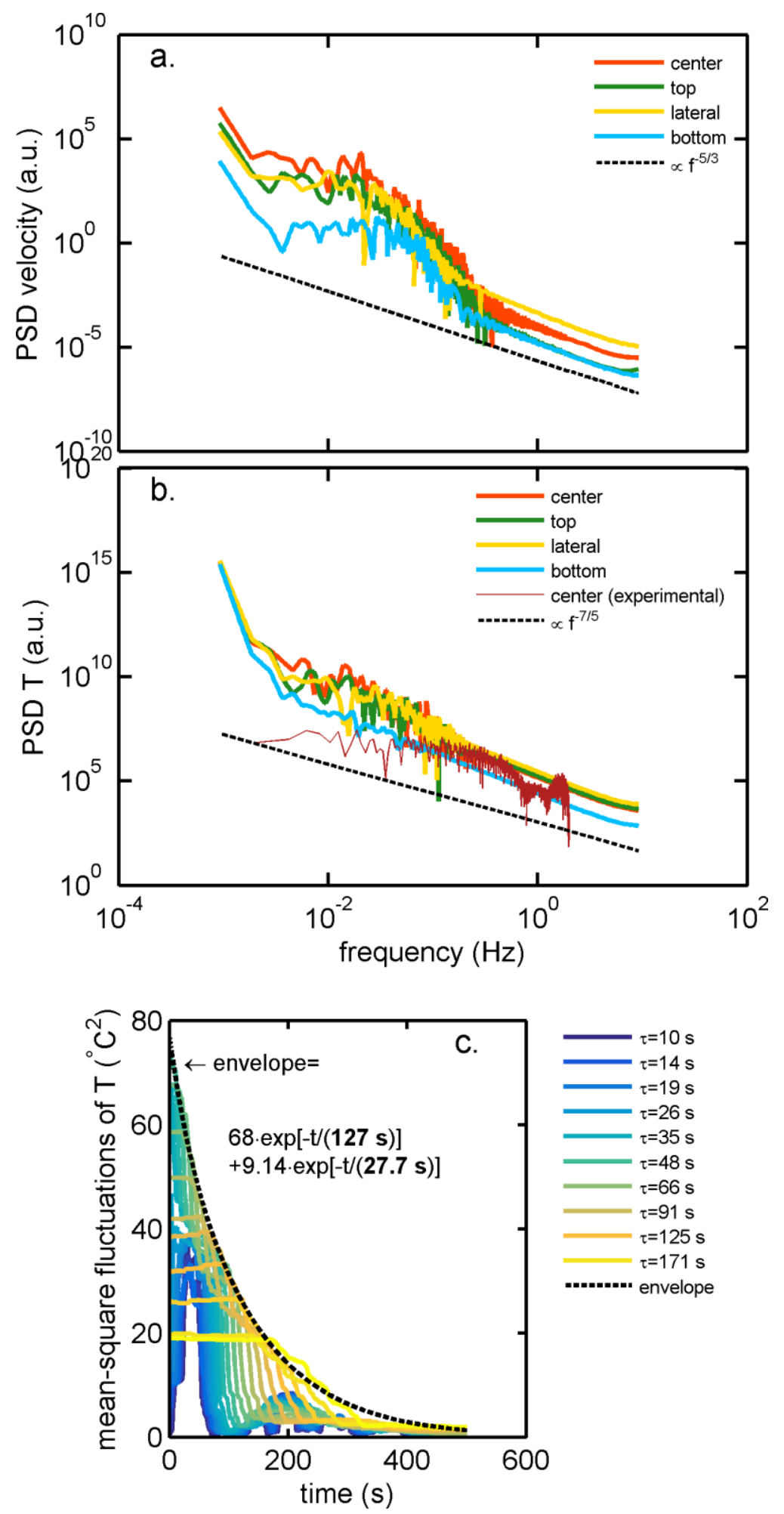

524 Figure 8. Analyses of fluctuations of (a) velocities and (b-c) temperatures. PSD. (a-b) Temporal power spectrum densities at different positions and comparison with its scaling as $f={ }^{-m}$ with $m=5 / 3$ for isotropic scaling [69] and $m=7 / 5$ for stratified scaling [70]. (c) Windowed mean-square fluctuations as measured at the center of the deep-fryer ( $\tau$ is the width of the window). 
529 At steady state, the natural convection is fully developed in the entire oil bath, as shown in 530 Figure 9. The spatial distribution of residence times was estimated on a regular grid of 531 elementary volumes $V_{r}$ from mass flow considering a compressible flow according to the 532 following equation:

$$
t_{r}(x, y, z)=\frac{\int_{V_{r}} \rho(x, y, z) d x d y d z}{\sum_{f=1 . .6} \int_{\partial \Omega_{f}}\left(\rho(x, y, z) \mathbf{U}(x, y, z) \cdot \mathbf{n}_{\partial \Omega_{f}}\right) \mathrm{H}\left[\mathbf{U}(x, y, z) \cdot \mathbf{n}_{\partial \Omega_{f}}\right] d \Omega_{f}}
$$

534 where $\mathbf{U}(x, y, z)$ is the velocity field at positions $(x, y, z), \rho(x, y, z)$ is the oil density at the

535 same positions calculated from the local temperature. $\left\{\partial \boldsymbol{\Omega}_{f}\right\}_{f=1 . .6}$ are the faces of the element 536 with normals $\mathbf{n}_{\partial \Omega_{f}}$ and $H$ the Heaviside function.

537 Local residence times shown in Figure 9a were calculated with a spatial resolution of $1.7 \mathrm{~mm}$. 538 They highlighted the presence of a central chimney were oil transited rapidly. Its presence was 539 confirmed by the direct observation of the flow (Figure 9b). The tracked particle shows that the 540 chimney compartmented recirculation loops on both sides of the cross section. Dwelling times 541 below the heating element reaches several minutes up to almost one hour with temperatures $54280^{\circ} \mathrm{C}$ lower than those observed above. As first approximation and in agreement with the overrepresentation of cold surfaces, residence times were significantly greater in cold regions

544 than in cold ones. Non-uniform residence times correlated with non-uniform temperature 545 distributions would extend the lifetime of hydroperoxides and confirmed our initial intuition 546 about the likely relationship between deep-fryer design and oil abuse. 
a.
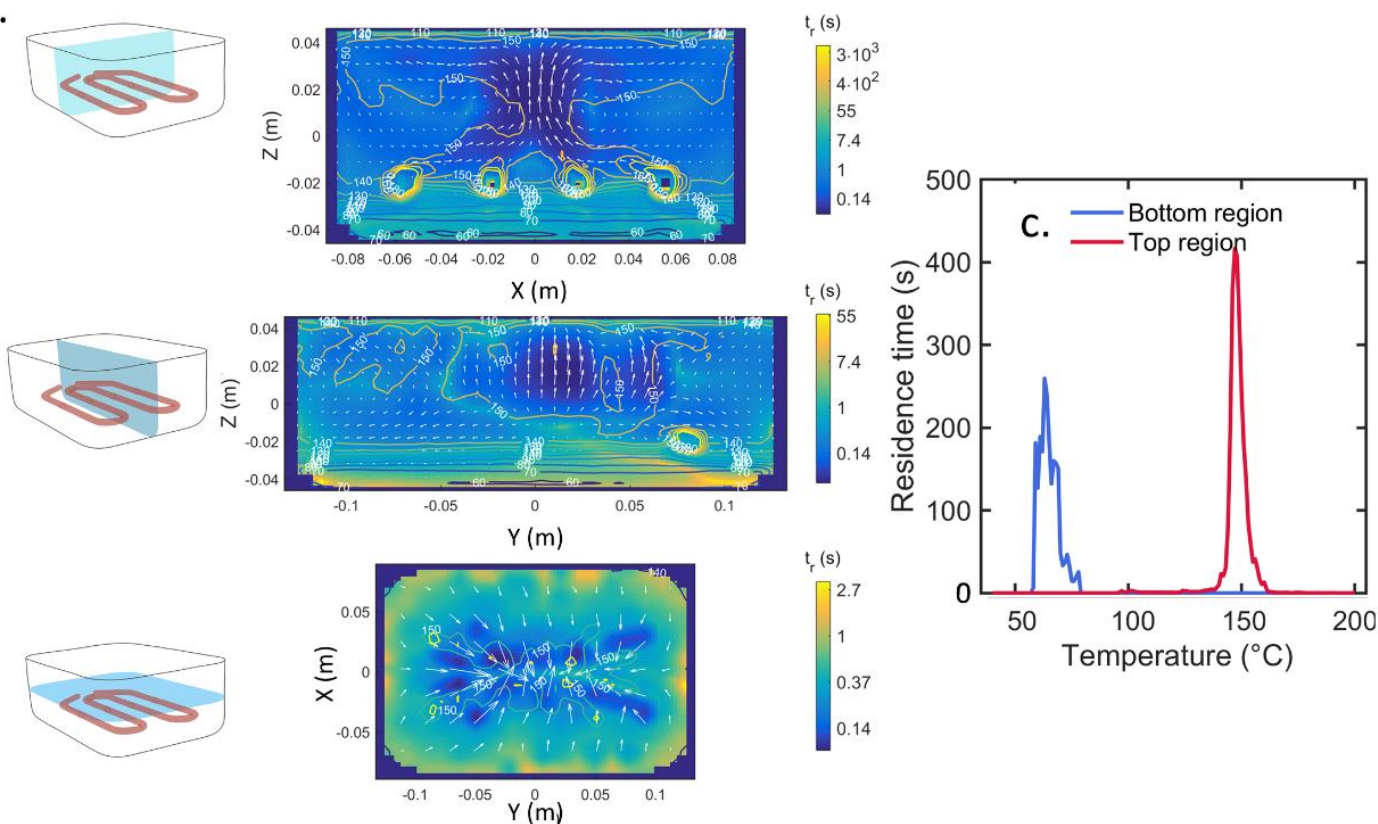

b.

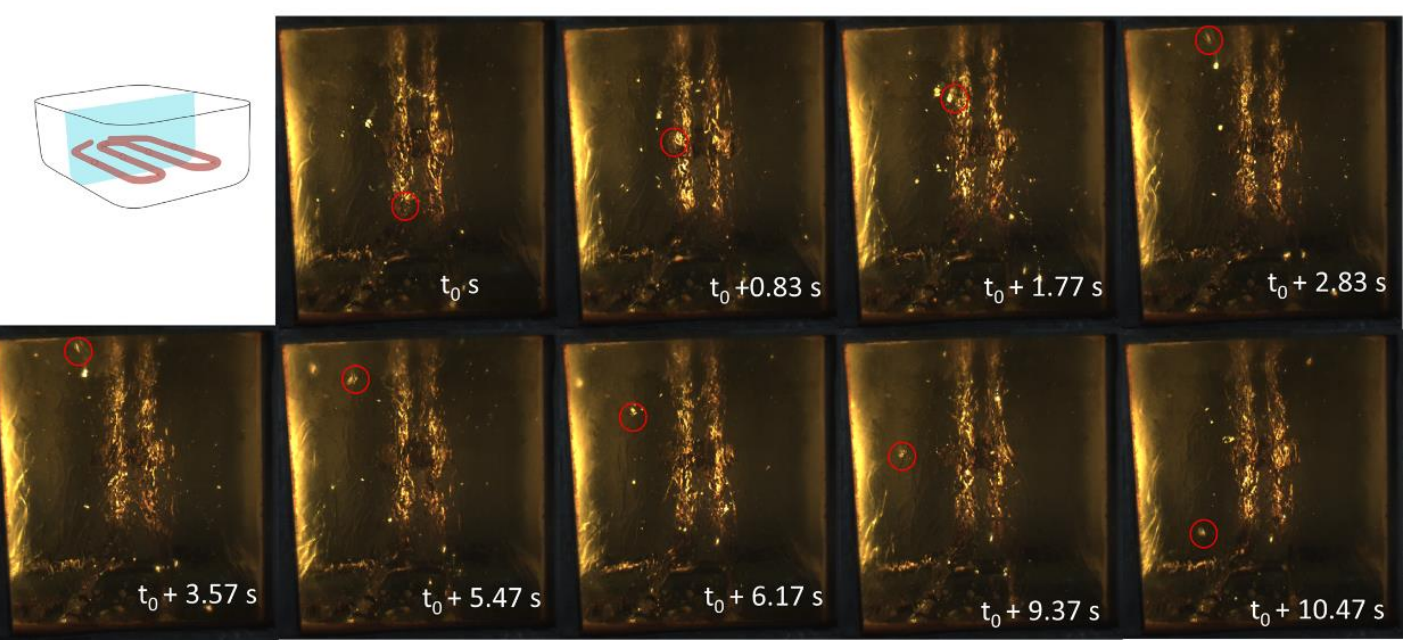

Figure 9. (a) Temperature (iso-contours), velocity (arrows) and residence time (color) fields at steady state in the principal planes of the deep-fryer (resolution of $1.7 \mathrm{~mm}$ ) and (b) experimental observations of scattered light at $90^{\circ}$ through the plane $\mathrm{XZ}$ at steady state. The amount of scattered light characterized temperature gradients and the presence advected particles ( 20 flat wood chip particles with a diameter of ca. 3-5 mm were dispersed in oil bath). 

decomposition in the deep-fryer

560 The transport of hydroperoxides from one region to another one was simulated at steady state

561 using a Lagrangian formulation. This description enables to simulate transport on time scales

562 (several hours) much longer than those use to simulate the flow velocity at steady state ( $\left.\mathbf{U}\right|_{x, y, z} ^{\text {steady }}$

563 ). The trajectory of fluid particle " $\alpha$ " containing hydroperoxides was integrated explicitly over 564 time, where the displacement $\Delta \mathbf{r}_{\alpha}$ during $\Delta t$ is given by:

$$
\left.\Delta \mathbf{r}_{\boldsymbol{\alpha}}\right|_{x, y, z}=\left.\mathbf{U}\right|_{x, y, z} ^{\text {steady }} \Delta t+\sqrt{\left\langle\left.\Delta \tilde{\mathbf{r}}_{\alpha}^{2}\right|_{x, y, z}\right\rangle} \underline{\underline{\mathbf{N}}}(\mathbf{0 , 1})
$$

566 where $\underline{\underline{\mathbf{N}}}(\mathbf{0 , 1})$ is a $3 \times 3$ diagonal matrix whose diagonal elements are random drawn from the

567 standard normal distribution. $\left\langle\left.\Delta \tilde{\mathbf{r}}_{\alpha}^{2}\right|_{x, y, z}\right\rangle$ is the mean-square displacement due to the random

568 contribution of turbulence, where angular brackets denote an ensemble average.

569 Following Taylor's theory, fluctuations of particle velocities were assumed to be governed by 570 a stationary random process so that $\left\langle\left.\Delta \tilde{\mathbf{r}}_{\alpha}^{2}\right|_{x, y, z}\right\rangle$ could be estimated from their local 571 autocorrelation function $\left.\rho_{\alpha}^{2}\right|_{x, y, z}(\tau)$ depending on lag $\tau$ but not on time $t$. From Eq. 12.148 of 572 Ref. [73] it reads:

$$
\left\langle\left.\Delta \tilde{\mathbf{r}}_{\alpha}^{2}\right|_{x, y, z}\right\rangle=\left.2\left\langle\left.\mathbf{u}\right|_{x, y, z} ^{2}\right\rangle \Delta t \int_{0}^{\Delta t}\left(1-\frac{\tau}{\Delta t}\right) \rho_{\alpha}^{2}\right|_{x, y, z}(\tau) d \tau
$$


574 where $\left\langle\left.\mathbf{u}\right|_{x, y, z} ^{2}\right\rangle$ is the variance of fluctuations. By choosing an integration time step sufficiently 575 small comparatively to the total correlation time $\left.\Lambda\right|_{x, y, z}=\left.\int_{0}^{+\infty} \rho_{\alpha}^{2}\right|_{x, y, z}(\tau) d \tau$, Eq. (17) becomes:

$$
\left\langle\left.\Delta \tilde{\mathbf{r}}_{\alpha}^{2}\right|_{x, y, z}\right\rangle=\left\langle\left.\mathbf{u}\right|_{x, y, z} ^{2}\right\rangle \Delta t^{2}
$$

577 In the central part of the deep-fryer where the flow rate was the highest, correlation time $\left.\Lambda\right|_{0,0,0}$ 578 was about $24 \pm 8 \mathrm{~s}$. As a result, choosing $\left.\Delta t \approx 0.1 \mathrm{~s} \ll \Lambda\right|_{0,0,0}$ justified that the contribution of 579 velocity fluctuations could be described as random ballistic trajectories (i.e., proportional to 580 time). The decomposition of hydroperoxides was simulated using a root mean square $581\left\langle\left.\mathbf{u}\right|_{x, y, z} ^{2}\right\rangle^{1 / 2}=\left.0.025 \mathbf{U}\right|_{x, y, z}$ (i.e., $95 \%$ of fluctuations $\pm 5 \%$ ), enabling smooth trajectories of 582 particles as experimentally observed in Figure 9b, while enabling particles to explore the entire 583 bath.

584 As a scalar field, temperature fluctuations are passively affected by the turbulent velocity field. 585 They decorrelated, therefore, almost similarly as velocity on time scales, $\Delta t$ (see Figure 9b), 586 where $L$ is the largest eddy scale, typically half-width. On time scales commensurable to $\Delta t$, 587 several complications complicated the sampling of thermal fluctuations as reported in Refs. 588 [74-77] In liquids with high Prandtl numbers as oil, intense sources and intermittently rising 589 plumes were responsible for strong memory effects, including the persistence of temperature 590 fronts and profiles mimicking the shape of heating elements (see Figures 5 and 9). In the sake 591 of simplicity, turbulent variations of temperatures were introduced in our Lagrangian 592 formulation by authorizing the visited temperature $\left.T\right|_{x, y, z}$ to fluctuate as a random white noise 593 normally distributed around 0 with a standard deviation proportional to $\frac{\left.T\right|_{x, y, z}-70}{T_{s k i n}-70} 5^{\circ} C$. The 
594 proposed noise scaling reproduced minimal fluctuations within the stagnant region and 595 maximal in heated ones as experimentally assessed.

596 Though hydroperoxides may be present at the beginning of the heating cycle in the oil bath, 597 they are continuously regenerated at the extreme surface. As a result, the trajectories of fluid 598 particles emitted from the surface are more relevant to envision their fate in the oil bath. Typical 599 fluid particle trajectories originating from the surface, in the median plane ( $Z=0$ ), and below 600 the heating element are shown in Figure 10. They correspond to a convective transport longer 601 than $1200 \mathrm{~s}$. The rapid recirculation from the heating element back to the free surface confirms 602 that an oil particle transporting hydroperoxides may be rapidly exposed again to atmospheric 603 oxygen, facilitating their renewal. The recirculation above the heating element much faster than 604 the decomposition rate of hydroperoxides (between $5 \cdot 10^{-4}$ and $1 \cdot 10^{-3} \mathrm{~s}^{-1}$ at $160^{\circ} \mathrm{C}$ ) justifies that 605 they are decomposed at a steady rate at frying temperature.

606 In detail, recirculation loops were similar whether the particles came from the surface (Figure 607 10a-d) or the bulk oil above the heating element (Figure 10e-h). Particles close to the vertical 608 symmetry plane $Y=0$ remained almost confined within the plane $X Y$. Outside the central 609 region, particles were oriented along the main cross-sections (diagonal planes), exploring all 610 corners. The merging of ascending plumes into one single central chimney forced the 611 convergence of trajectories towards the symmetry axis $X=Y=0$. The outside branches 612 followed the shape of the oil bath and the symmetry planes while avoiding the inner part of the 613 loops. This configuration maximizes the exposure to high temperature (path along the heating 614 resistor) and oxygen (path along the free surface). Mixing times were evaluated using the 615 concept of random walks in Figure 10m and defined as the average mixing time $(\tau)$ required 616 to get a mean-square displacement (MSD) equal to the surface area of the explorable section. 617 Mixing was said isotropic when MSD grew similarly along the main axes of the cross-section. 
618 By analogy with diffusion on a line of length $L$, the mixing length along direction $d$ is given

619 by $6 \max \left(M S D_{\text {along } d}\right)$. Along direction X and Z, MSD increased proportionally with vising

620 time (parabolic with $\sqrt{\tau}$ ) in a very similar manner in less than $200 \mathrm{~s}$. This behavior was typical

621 of uncorrelated displacements and corresponded to a maximum visited distance of $67 \pm 3 \mathrm{~mm}$,

622 which was periodically achieved approximately every $460 \pm 20$ s. Along Y, displacements

623 followed ballistic trajectories proportional to $\tau$. Forty-minutes were required to visit half-

624 length of the deep-fryer without reaching a perfect mixing, while it was obtained in almost 3

625 minutes along the half-section of $\mathrm{XZ}$.

626 The incursions under the heating element from the upper part of the bath remained exceptional

627 and brief (see Figure 10c). The particles under the heating element move essentially

628 horizontally at a very low speed due to pressure fluctuations. The difference in viscosity on

629 both sides prevents mixing. The zone is stagnant (Figure 10i-1) and does not exhibit any

630 significant turnover under simulated conditions (first ten minutes of heating). These conditions

631 are responsible for the long duration (two hours) needed to reach a steady thermal regime under

632 the heating resistor. Lower temperatures during the first ten minutes exacerbated dead volume

633 effects. 
a.

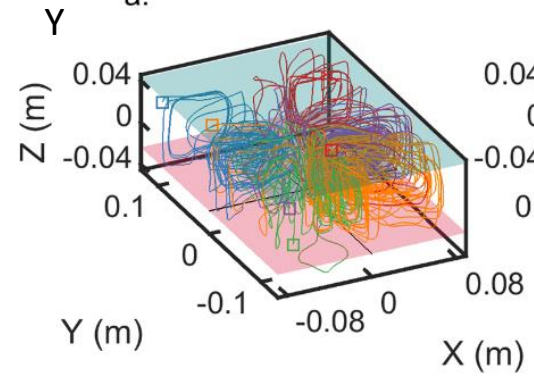

e.

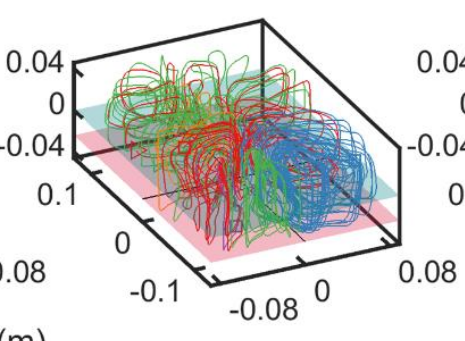

i.

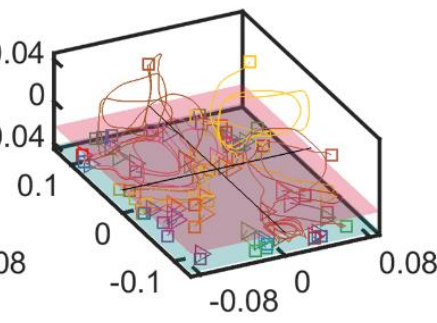

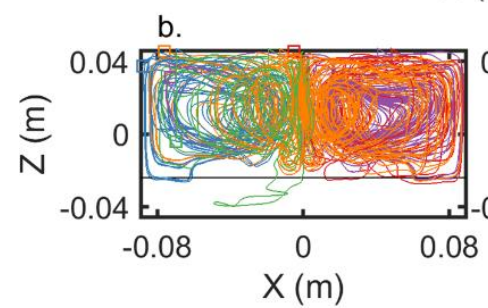
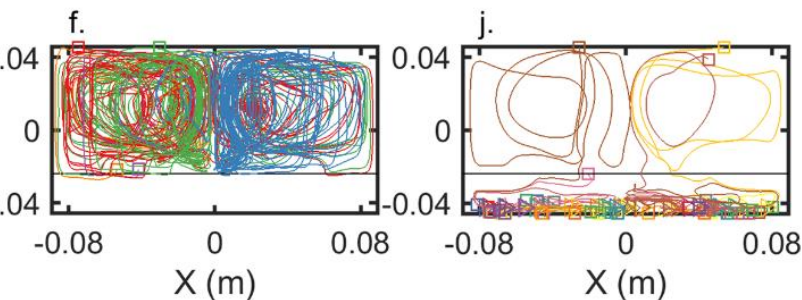

c.

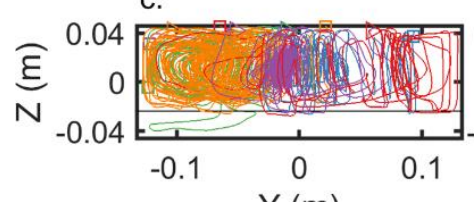

g.

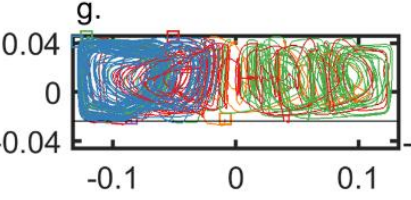

k.
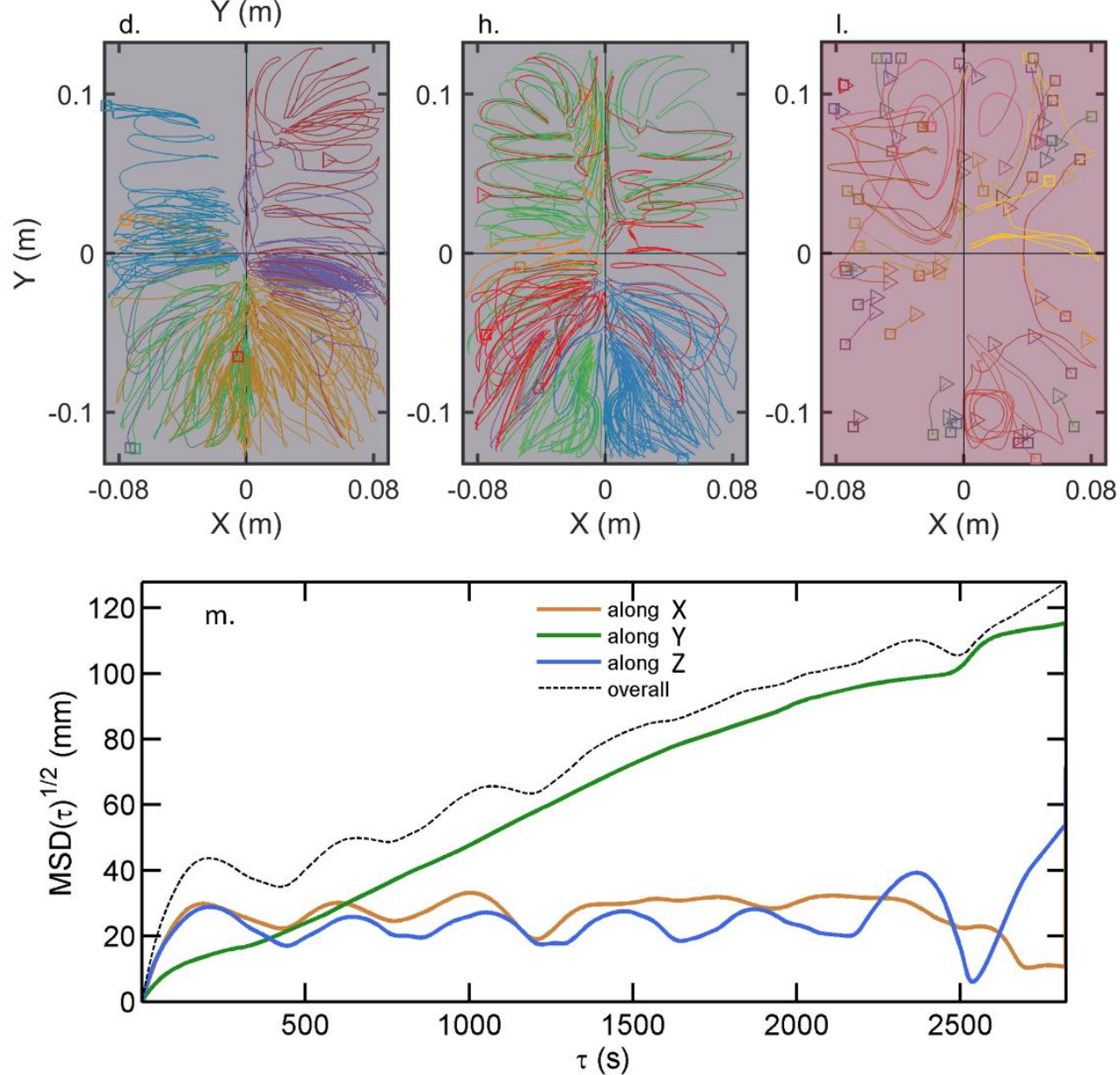

$\tau(\mathrm{s})$

Figure 10: Simulated trajectories originated from (a-d) the surface ( 5 trajectories), (e-h) the median plane ( $\mathrm{Z}=\mathbf{0}, 5$ 
640 The vertical distributions of particles on short and long-time scales are plotted in Figure 11,

641 based on the sampling of more than $3 \times 10^{3}$ trajectories starting from the top, median, and bottom

642 positions. On short time scales, the vertical dispersion was faster from the median position than

643 from top and bottom ones. Residence times were indeed much longer at the free surface and

644 bottom (see Figure 10). On longer time scales, dispersions were similar from top and median

645 positions almost uniform, but with large fluctuations in time. Vertical mixing times were above

646 ca. $10^{2} \mathrm{~s}$ and beyond $10^{4} \mathrm{~s}$ above and below the heating element, respectively. As a result, only

$6472 \pm 1 \%$ of particles initially present in the cold zone could reach the region above (Figure 11f).

648 The risk of accumulation of hydroperoxides in the cold zone by convection was low during

649 initial heating and temperature holding. On the corollary, hydroperoxides present at the

650 beginning of heating would decompose very slowly in the stagnant region without escaping.

651 Without mixing and in extreme anoxia, polymerization and cyclization termination reactions

652 (exothermic) will dominate, whereas scission reactions (endothermic) would dominate

653 immediately above the heating element due to much higher temperatures and rapid renewal.

654 The production of volatile compounds far from the surface, where they are finally desorbed,

655 has been previously characterized by local sampling [10]. 

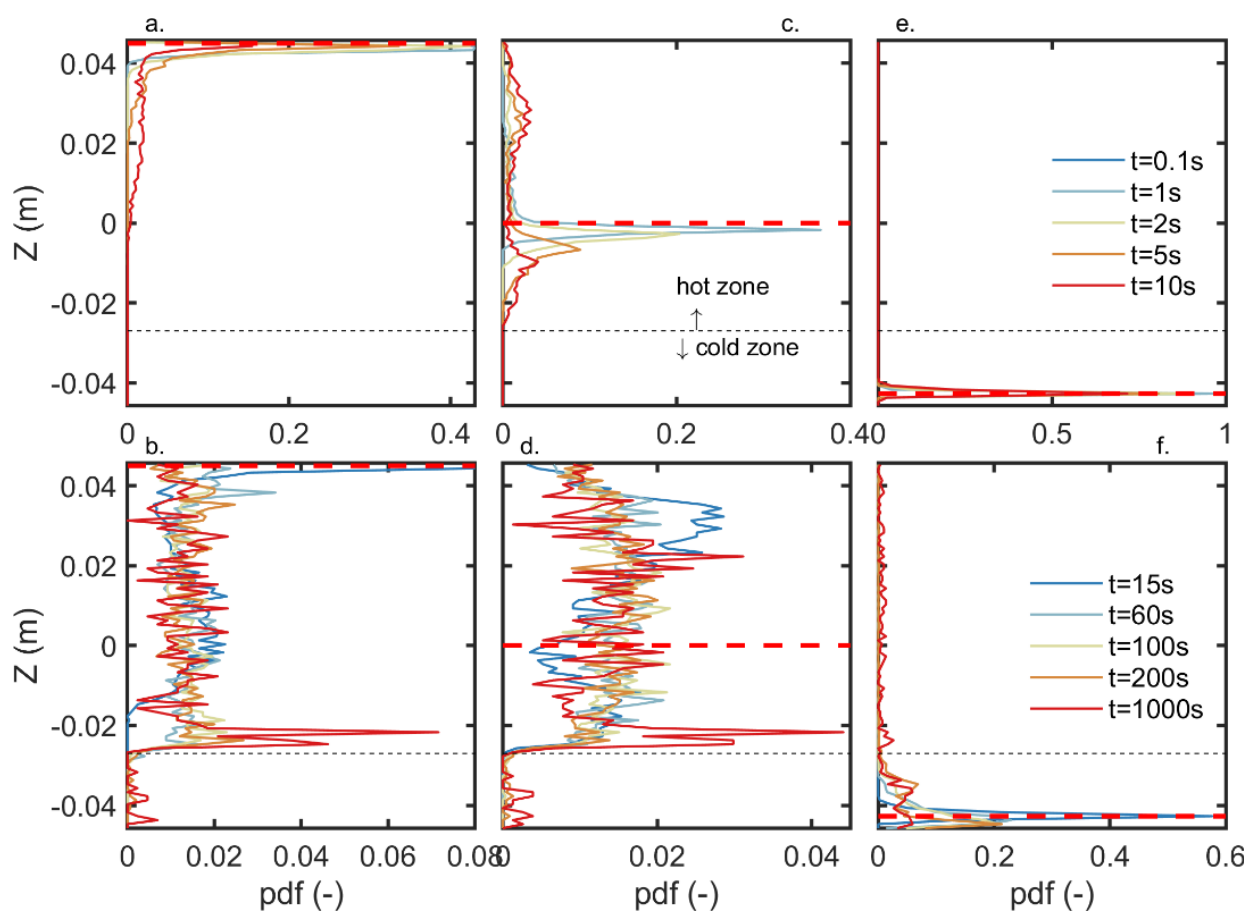

Figure 11: position distributions of oil particles with time according to the starting point from the surface (a-b), the median plan $(c, d)$ and under the heating element (e,f). The red dotted lines represent the initial position of the particles along Z.

661 The decomposition of hydroperoxides in anoxia was simulated during four hours, starting from 662 a uniform initial distribution in the deep-fryer, mimicking a real production cycle using

663 sunflower oil. The effective decomposition for the regions above and below the heating element 664 are compared with experimental values inFigure 12. The initial hydroperoxides were generated by bubbling air at $140^{\circ} \mathrm{C}$ (this temperature was shown to maximize hydroperoxide production

666 in a reasonable time according to Ref. [11]). The initial composition in hydroperoxides was

667 reconstructed from Ref. [11]. Experimentally, only the total amount of hydroperoxides was 668 measured by FTIR [10]. The concentration in hydroperoxides was integrated along $1.2 \cdot 10^{3}$ 669 trajectories initiated from equispaced points distributed uniformly via Eq.(11) and calculated 670 independently with Eq.(12) from the distribution of residence times in temperature in the bath 
as shown in Figure 12a. The separation between upper and lower regions was artificially imposed by the starting position, but the exchange between regions was permitted.

673 For the upper volume, single-particle tracking (denoted PT) and residence time (denoted RT)

674 approaches are theoretically equivalent for durations longer than mixing time $(\sim 3$ min along

$675 \mathrm{XY}$ ). In this case, the exploration of regions at different temperatures is independent of the

676 order in which they are visited. Residence times depicted in Figure 12a were sampled from

677 random walks initiated from the upper region to avoid sampling biases due to hydroperoxides

678 decomposing faster than the time of passages from one region to the next (see justification in

679 the general discussion of Ref. [78] ). The distribution for particles originating from arbitrary

680 positions is shown in Figure 9c. Only the PT approach gives access to the variability within the deep-fryer due to dead volumes or overheated regions close to the heating element. More than $60 \%$ of PT values were indiscernible from RT determinations. Only three values were

683 significantly above and corresponded trajectories reaching the bottom region underneath the

684 heating element. Lower values were comparatively more frequent and were observed as soon 685 as the trajectory approached the heating element (with temperatures above $160^{\circ} \mathrm{C}$ ), leading to 686 much faster decomposition. The detailed reconstruction for each hydroperoxides showed that, 687 though hydroperoxides from oleic $\left(\mathrm{L}_{1} \mathrm{OOH}\right)$ and linoleic fatty esters $\left(\mathrm{L}_{2} \mathrm{OOH}\right)$ were produced 688 in almost similar amounts in the bubbling reactor, the second decompose four times faster. $689 \mathrm{~L}_{2} \mathrm{OOH}$ were all depleted in one hour whereas only half of $\mathrm{L}_{1} \mathrm{OOH}$ was. During a typical 690 production cycle of one hour (heating, temperature holding, frying, cooling), these results 691 would demonstrate that $\mathrm{L}_{2} \mathrm{OOH}$ would responsible for most of the inconvenience (smell, 692 fouling, loss of nutritional value) whereas $\mathrm{L}_{1} \mathrm{OOH}$ would certainly reinitiate the oxidation of 693 lipids during the next production cycle. 
694 For the lower region, thermal equilibration times ( 2 hours) much longer than simulated heating 695 times $(10 \mathrm{~min})$ make simulated temperatures $\left(\sim 70^{\circ} \mathrm{C}\right)$ unsuitable for predicting the 696 decomposition of hydroperoxides. A constant temperature of $135^{\circ} \mathrm{C}$ close to experimental 697 measurements $\left(135 \pm 3^{\circ} \mathrm{C}\right)$ was applied instead. Since the region was uniform in temperature, no 698 PT results are shown. It is nevertheless underlined that the displacements are slow in the 699 stagnant region (see Figure 101). Since the exchange between upper and lower regions was 700 minimal, the proposed approximation led to excellent predictions below the resistance during 701 the two first hours. Beyond two hours, their amount was overestimated due to a significant mass 702 transfer loss by diffusion and advection. 

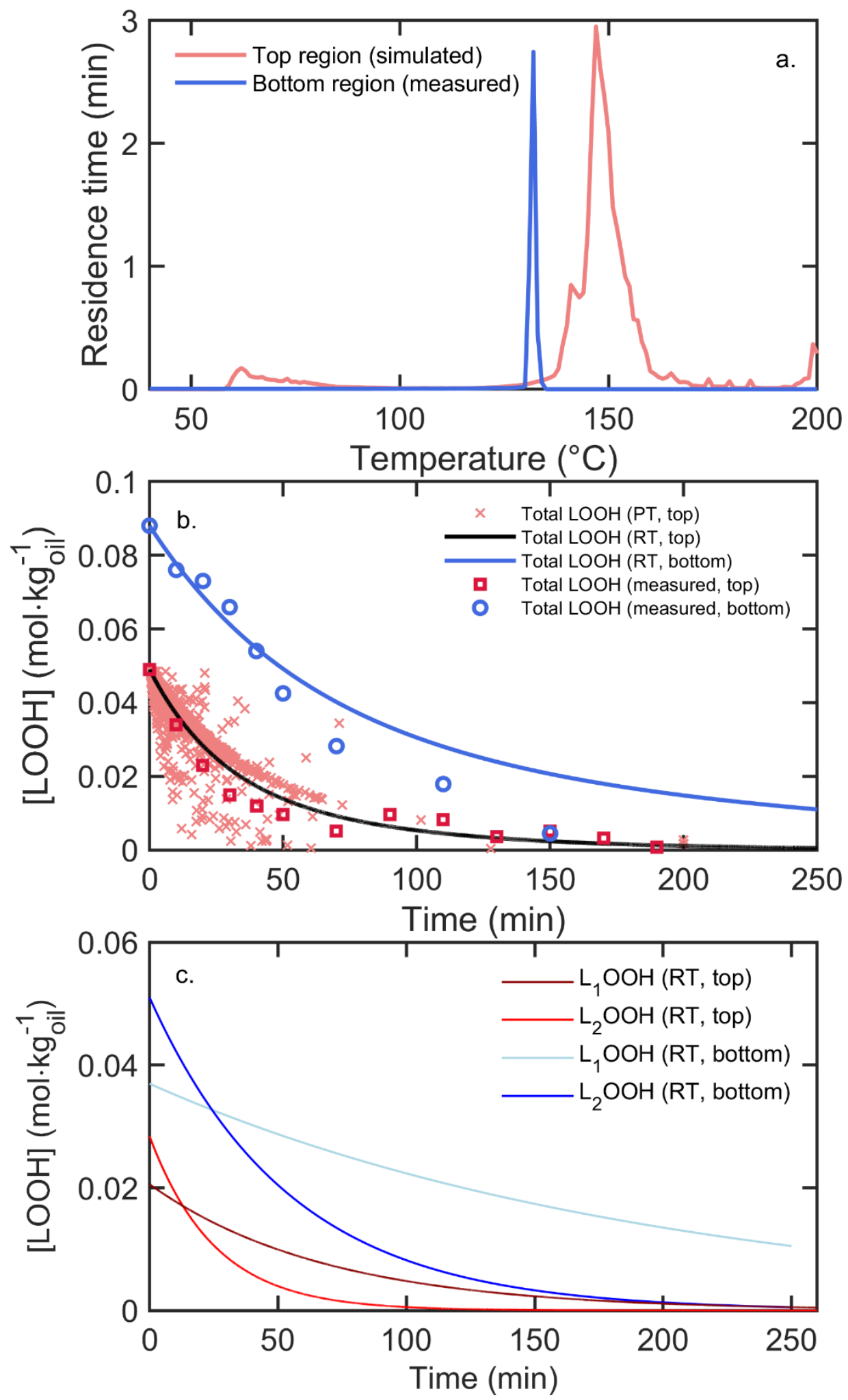

Figure 12: Residence time at each temperature calculated for 1200 trajectories (a) and comparison between measured and predicted values of $\mathrm{LOOH}$ concentration in the top and bottom regions (b) 
709 Since the lifetime of hydroperoxides is longer than vertical mixing times, the distribution of

710 residence times and temperatures govern the kinetics of decomposition of hydroperoxides.

711 Figure 13 shows the oil flow structure and residence times within a deep-fryer directly heating

712 from a rounded bottom and a limited cold zone. Heating elements are located at the top-end and

713 at the bottom of the rounded part of the tank. Depicted simulations showed similar temperatures

714 averaged over the entire oil volume but with different distributions (Figure 13b). The theoretical

715 effects of the decomposition of hydroperoxides is shown in Figure 13c. In de depicted

716 configuration, the new design without cold zone doubled the effective lifetime of

717 hydroperoxides. The effects could not be guessed without simulation as some effects are

718 antagonist. The modified configuration introduced hot spots, but also accelerated the dispersion

719 in a larger accessible volume. The higher efficiency originates from the rounded bottom

720 performing the recirculation and the creation of three independent plumes. 
a.

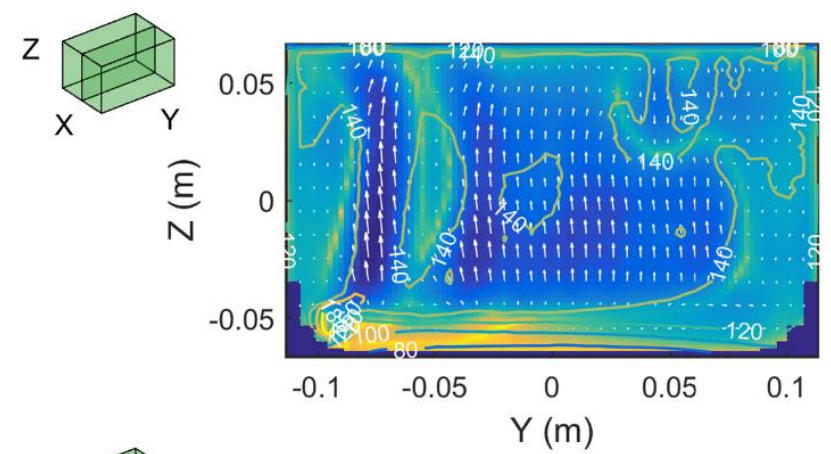

Z

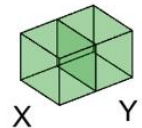

z
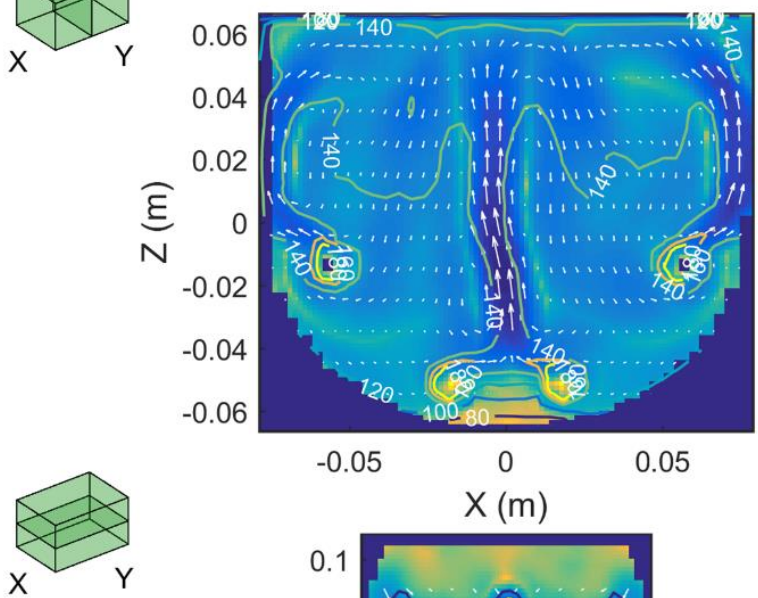

722

724

725

726

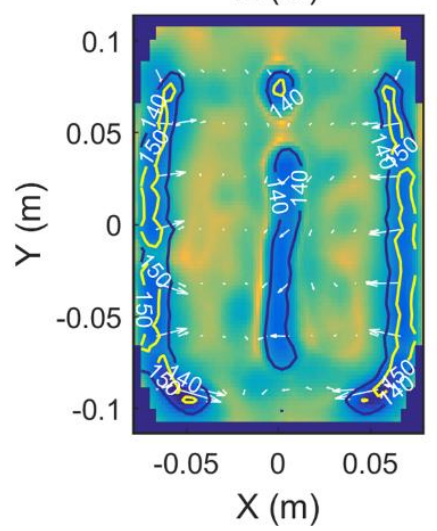
hydroperoxides decomposition. b.
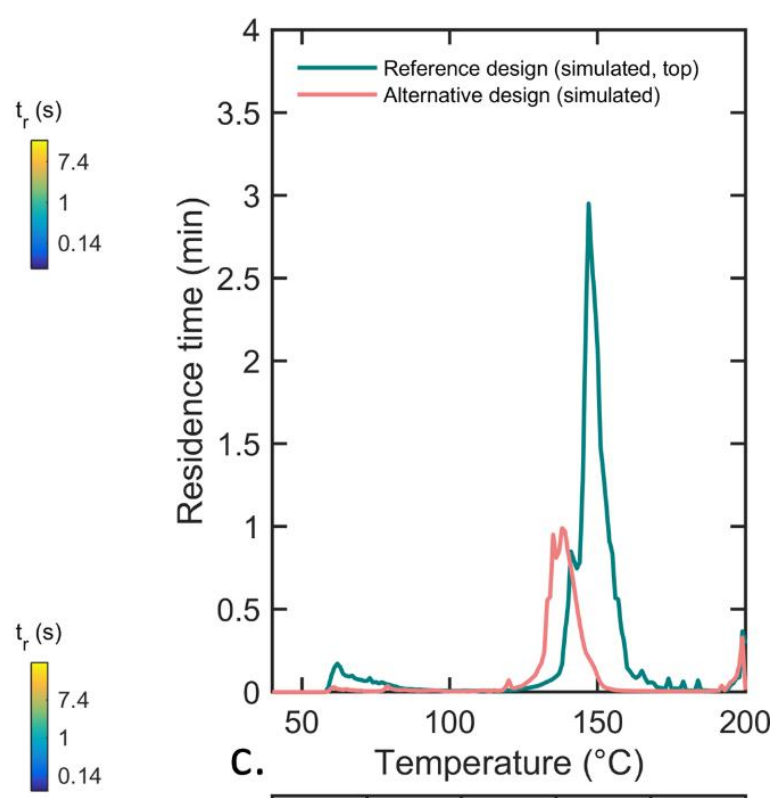

C.

Temperature $\left({ }^{\circ} \mathrm{C}\right)$
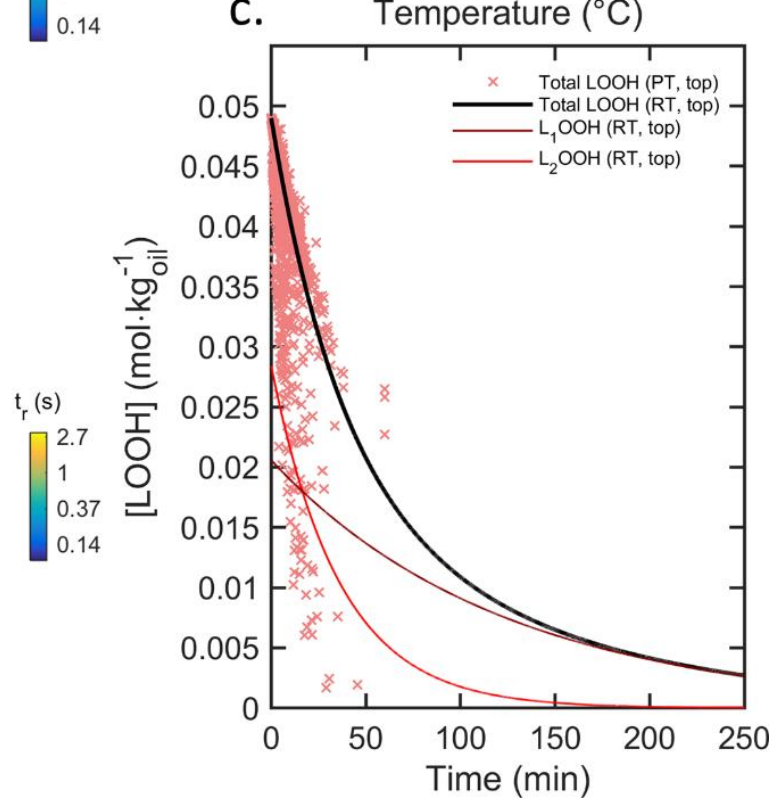

Figure 13. (a) Anisothermal oil flow within a $4 \mathrm{~L}$ deep-fryer without cold zone and equipped with four heating coils (two at the bottom and two at the transition between the rounded bottom and the vertical walls). (b) Comparison of temperature residence times with the reference design (see Figure 9) at steady-state and (c) corresponding

\section{Conclusions}

728 Autoxidation of triacylglycerols during frying has previously been studied as a chemical problem without considering temperature spatial heterogeneities and the coupling with vertical recirculation of oil between the heating element and the free surface. The flow is structured 
during the first minutes of heating with fluctuations that dissipate exponentially with the

732 decrease of temperature difference between the hottest and coldest regions. The heating of the

733 oil under the electrical element coils is, conversely, delayed by almost two hours. The stationary

734 temperature is $30-40^{\circ} \mathrm{C}$ below the frying temperature set above the heating element.

735 Temperaure fluctuations $\left( \pm 3^{\circ} \mathrm{C}\right)$ in the frying region are much lower than spatial heterogeneities

$73670-80^{\circ} \mathrm{C}$ with strong variations of residence times of fluid parcels. At steady state, CFD

737 simulations showed rapidly rising plumes rapidly reorienting along the free surface where they

738 cooled before beginning their slow subduction. This process disperses triacylglycerols and their

739 hydroperoxides in almost of one quarter of the deep-fryer. The merging of several plumes in

740 the central region generates a strong updraft forcing periodic exposure of hydroperoxides to

741 high temperatures with pseudo-periods ranging from 3 to 5 minutes. The orientation of

742 streamlines parallel to the free surface favors the dissolution of oxygen and thus the formation

743 of hydroperoxides. The lifetime of hydroperoxides depends strongly on parent fatty esters at

744 low and intermediate temperatures but in a less extent at frying temperatures. The presence of

745 cold regions is essential to the preservation of unsatured fatty esters, with the highest nutritional

746 value. By contrast, hot surfaces destroy without differentiation all fatty esters. Transfer across

747 the symmetry planes and mass transfer between the cold and the frying regions remains 748 infrequent.

749 Since the time scales of hydroperoxide decomposition and vertical mixing times are separable,

750 the decomposition kinetics of hydroperoxides and the rate of oxidation can be reconstructed

751 from the distribution of residence times at different temperatures. This simplifying assumption

752 facilitates the exploration of the relationship between fryer design and oil life by CFD

753 simulations. The principles of a concurrent design methodology were outlined for two

754 immersion fryers with a single heating plane in a parallelepipedic tank (standard design) and in

755 a tank with a rounded bottom and two heating planes. Increasing the oil height and reducing the 
cross-sectional area reduces the exposure to oxygen and extends the useful volume for frying.

757 Larger volumes reduce the need for extreme temperatures. Current analyses do not take into 758 account the presence of the basket and the product which can not only modify the structure of

759 the flow and the product. Preliminary studies (results not shown) have shown that elements that

760 obstruct the vertical flow are responsible for vertical temperature stratification and hot spots.

761 This study demonstrates the strong spatialization of oxidation radical reactions. The upward

762 velocities although fast in the central part of the fryer are insufficient to justify a transport of 763 highly unstable alkoxyl, hydroperoxyl and alkyl radicals (lifetimes of the order of

764 microseconds) from the heating elements to the free surface. The decomposition of 765 hydroperoxides in depth and in anoxia produces essentially scission compounds in competition 766 with the polymerization reactions responsible for the fouling of the heating surfaces. The 767 coupling between the production of voluble organic compounds by scission, their transport and 768 their desorption at the surface will be detailed in a companion paper.

\section{Acknowledgements}

771 The authors would like to thank the project Investissements d'Avenir FUI-AAP17 "Fry'In", 772 which contributed to fund a part of the study. Mathilde Blondel (SEB) and Laurent Berthier 773 (Lesieur) are particularly thanked for supplying the deep-fryers and the vegetable oil, 774 respectively.

\section{Declaration of interest}

777 The authors declare no conflict of interest. 


\section{Data availability statement}

780 The data that support the findings of this study are available from the corresponding author

781

upon reasonable request.

782

783

\section{Appendix A: Simplified oxidation model in mixture}

784

785

786

787

As the propagation via alkoxyl and hydroxyl radicals and oxygen addition are several magnitudes faster than the hydrogen abstraction via peroxyl radicals and hydroperoxide decomposition [19], the lumped mass balance reads:

$$
\begin{aligned}
& \frac{d\left[L_{i} O O H\right]}{d t}=-k_{d_{i}}(T)\left[L_{i} O O H\right]+k_{p_{i}}\left(T,\left[O_{2}\right]\right)\left[L_{i} H\right] \\
& \frac{d\left[L_{i} H\right]}{d t}=-k_{p_{i}}\left(T,\left[O_{2}\right]\right)\left[L_{i} H\right]
\end{aligned}
$$

788 where $\left\{k_{d_{i}}\right\}_{i=1,2,3}(T)$ and $\left\{k_{p_{i}}\right\}_{i=1,2,3}\left(T,\left[O_{2}\right]\right)$ are the temperature dependent reaction rates of

789 decomposition and apparent propagation, respectively. By definition, the apparent reaction rate $790 \quad k_{p_{i}}=0$ when $\left[O_{2}\right]=0$.

791 Simplified model (Eq. (7)) is applicable to any isothermal dataset in anoxia and in oxygenated 792 conditions if $k_{p_{i}}$ constants are determined independently. Following Refs. [79, 80], the equality $793 k_{p_{3}}=2 k_{p_{2}}$ holds.

\section{References}

795 [1] M. Touffet, G. Trystram, O. Vitrac, Revisiting the mechanisms of oil uptake during deep796 frying, Food and Bioproducts Processing, 123 (2020) 14-30.

797 [2] J.-M. Vauvre, A. Patsioura, R. Kesteloot, O. Vitrac, Multiscale modeling of oil uptake in 798 fried products, AIChE Journal, 61(7) (2015) 2329-2353. 
[3] C. Schneider, N.A. Porter, A.R. Brash, Routes to 4-hydroxynonenal: Fundamental issues in the mechanisms of lipid peroxidation, Journal of Biological Chemistry, 283(23) (2008) 1553980115543.

802 [4] D.S. Mottram, B.L. Wedzicha, A.T. Dodson, Acrylamide is formed in the Maillard reaction, 803 Nature, 419(6906) (2002) 448-449.

804 [5] European Commission, COMMISSION REGULATION (EU) 2017/2158 of 20 November 8052017 establishing mitigation measures and benchmark levels for the reduction of the presence 806 of acrylamide in food, Official Journal of the European Union, L304 (2017) 24-44.

807 [6] F.D. Gunstone, Vegetable Oils in Food Technology, 1st ed., Blackwell Publishing, Osney 808 Mead, Oxford OX2 0EL, UK, 2002.

809 [7] M. Asif, Health effects of omega-3,6,9 fatty acids: Perilla frutescens is a good example of 810 plant oils, Oriental Pharmacy \& Experimental Medicine, 11(1) (2011) 51-59.

811 [8] A.P. Simopoulos, The importance of the ratio of omega-6/omega-3 essential fatty acids, 812 Biomedicine \& pharmacotherapy = Biomedecine \& pharmacotherapie, 56(8) (2002) 365-379.

813 [9] E.N. Frankel, Volatile lipid oxidation products, Progress in Lipid Research, 22(1) (1983) 181433.

815 [10] M. Touffet, A. Patsioura, A.M. Ziaiifar, L. Eveleigh, O. Vitrac, Online reconstruction of 816 oil oxidation kinetics and reaction schemes during deep-frying by deconvolution of ATR-FTIR 817 spectra, Journal of Food Engineering, 224 (2018) 1-16.

818 [11] A. Patsioura, A.M. Ziaiifar, P. Smith, A. Menzel, O. Vitrac, Effects of oxygenation and 819 process conditions on thermo-oxidation of oil during deep-frying, Food and Bioproducts 820 Processing, 101 (2017) 84-99.

821 [12] A. François-Heude, E. Richaud, A. Guinault, E. Desnoux, X. Colin, Impact of oxygen 822 transport properties on the kinetic modeling of polypropylene thermal oxidation. II. Effect of 823 oxygen diffusivity, Journal of Applied Polymer Science, 132(6) (2015).

[13] S.P. Rock, Roth, H, Factors Affecting the Rate of Deterioration in the Frying Qualities of Fats. 2. Type of heater and method of heating., Journal of the American Chemical Society, 48(8) (1964) 531-533.

[14] S.P. Rock, Roth, H, Factors Affecting the Rate of Deterioration in the Frying Qualities of Fats. 1. Exposure to air, The Journal of the American Oil Chemists' Society, 41 (1964) 228 230.

[15] O. Vitrac, G. Trystram, A method for time and spatially resolved measurement of convective heat transfer coefficient (h) in complex flows, Chemical Engineering Science, 60(5) (2005) 1219-1236.

[16] O. Vitrac, G. Trystram, A.-L. Raoult-Wack, Continuous measurement of convective heat flux during deep-frying: validation and application to inverse modeling, Journal of Food Engineering, 60(2) (2003) 111-124.

[17] W.T. Borden, R. Hoffmann, T. Stuyver, B. Chen, Dioxygen: What Makes This Triplet Diradical Kinetically Persistent?, Journal of the American Chemical Society, 139(26) (2017) 9010-9018.

[18] A.V. Tobolsky, D.J. Metz, R.B. Mesrobian, Low Temperature Autoxidation of Hydrocarbons: the Phenomenon of Maximum Rates1,2, Journal of the American Chemical Society, 72(5) (1950) 1942-1952.

[19] K.M. Schaich, Challenges in Elucidating Lipid Oxidation Mechanisms. When, Where, and How Do Products Arise ?, in: A. Logan, U. Nienaber, X. Pan (Eds.) Lipid Oxidation, AOCS, 2013, pp. 1-51.

[20] J.L. Bolland, P. ten Have, Kinetic studies in the chemistry of rubber and related materials. VII. The mechanism of chain propagation in the oxidation of polyisoprenes, Transactions of the Faraday Society, 45(0) (1949) 93-100. 
[21] J.L. Bolland, G. Gee, Kinetic studies in the chemistry of rubber and related materials. II. The kinetics of oxidation of unconjugated olefins, Transactions of the Faraday Society, 42(0)

$850 \quad$ (1946) 236-243. [22] J.L. Bolland, G. Gee, Kinetic studies in the chemistry of rubber and related materials. III. Thermochemistry and mechanisms of olefin oxidation, Transactions of the Faraday Society, 42(0) (1946) 244-252.

[23] J. Śliwiok, T. Kowalska, W. Kowalski, A. Biernat, The influence of hydrogen-bond association on the destruction of hydroperoxides in the autoxidation process of oleyl alcohol, oleic acid, and methyl oleate, Microchemical Journal, 19(4) (1974) 362-372.

[24] T. Nishiike, H. Takamura, T. Matoba, Stability of Linoleic Acid Hydroperoxide in the Oil System, Journal of Cookery Science of Japan, 33(3) (2000) 377-380.

861

862

863

864

865

866

867

868

869

870

871

872

873

874

875

876

877

878

879

880

881

882

883

884

885

886

887

888

889

[25] Y. Kawano, H. Nakatake, Y. Hatate, Degradation of fats and oils by autoxidation VI : Production and decomposition rates of hydrperoxide during the autoxidation of trioleil, (1989) 87-91.

[26] Y. Hatate, Y. Kawano, H. Sone, Autoxidation rates of oleic-acid and oleates, Journal of Chemical Engineering of Japan, 22(4) (1989) 407-412.

[27] A.J. Shin, D.H. Kim, A Mechanism for The hydroperoxyde Decomposition in a Soybean oil during Thermal Oxidation, Korean Journal Food Science Technology, 17(2) (1985) 71-74.

[28] I. Erkilla, T. Fung, M. Kandiah, J. Wilkins, J.J. Moran, J.A. Blake, Study of the accelerated oxidation of low and high erucic rapeseed oil, Journal of the American Oil Chemists' Society, 55(3) (1978) 303-309.

[29] J. Pokorny, J. Rzepa, G. Janicek, Lipid Oxidation. Part.1. Effetc of Free Carboxyl Group on the Decomposition of Lipid Hydroperoxide, Molecular Nutrition Food Research, 20(1) (1976) 1-6.

[30] O.S. Privett, Autoxidation and autoxidative polymerization, Journal of the American Oil Chemists Society, 36(10) (1959) 507-512.

[31] V. Stannett, A.E. Woodward, R.B. Mesrobian, Autoxidation of 1,4-Dimethylcyclohexane, The Journal of Physical Chemistry, 61(3) (1957) 360-364.

[32] J.R. Thomas, The Thermal Decomposition of Alkyl Hydroperoxides1, Journal of the American Chemical Society, 77(1) (1955) 246-248.

[33] L. Bateman, H. Hughes, A.L. Morris, Hydroperoxide decomposition in relation to the initiation of radical chain reactions, Discussions of the Faraday Society, 14(0) (1953) 190-199. [34] E.R. Bell, J.H. Raley, F.F. Rust, F.H. Seubold, W.E. Vaughan, Reactions of free radicals associated with low temperature oxidation of paraffins, Discussions of the Faraday Society, 10(0) (1951) 242-249.

[35] V. Stannett, R.B. Mesrobian, The Kinetics of the Decomposition of Tertiary Hydroperoxides in Solvents 1,2, Journal of the American Chemical Society, 72(9) (1950) 41254130.

[36] L. Bateman, H. Hughes, 895. The thermal decomposition of cyclohexenyl hydroperoxide in hydrocarbon solvents, Journal of the Chemical Society (Resumed), (0) (1952) 4594-4601.

[37] Y. Kawano, H. Sone, Y. Hatate, Autoxidation Rate of Methyl Oleate Diluted by Methyl Laureate, Journal of Chemical Engineering of Japan, 22(6) (1989) 703-705.

890 [38] Y. Hatate, Y. Kawano, T. Makise, A. Ikari, Degradation of fats and oils by autoxidation 891 III - Production and decomposition rates of hydroperoxide during the autoxidation of ethyl 892 oleate, Research Reports of the Faculty of Engineering, (28) (1986) 171-177.

893

894

895 [39] Y. Mochida, T. Yamada, Effects of Metal Chlorides of Periodic Group V, VI, and VII on the Autoxidation of Methyl Oleate and the Decomposition of Methyl Oleate Hydroperoxide, Journal of Japan Oil Chemists' Society, 34(7) (1985) 539-544. 
[40] Y. Mochida, T. Yamada, Effects of oxides and Chlorides of Metal of Periodic Groups 5, 6 and 7 on the Autoxidation of Methyl Oleate abd the Decomposition of Hydroperoxides of Methyl Oleate, Journal of Japan Oil Chemists'Society, 24(2) (1975) 107-111. [41] L. Bateman, Olefin oxidation, Quarterly Reviews, Chemical Society, 8(2) (1954) 147-167. [42] E. Choe, D.B. Min, Chemistry of Deep-Fat Frying Oils, Journal of Food Science, 72(5) (2007) 77-86.

[43] M.H. Brodnitz, Autoxidation of Saturated Fatty Acids. A Review, Journal of Agricultural and Food Chemistry, 16(6) (1968) 994-999.

[44] E.N. Frankel, Hydroperoxide formation, in: E.N. Frankel (Ed.) Lipid Oxidation, Woodhead Publishing Limited, 1518 Walnut Street, Suite 1100, Philadelphia, PA 19102-3406, USA, 2012, pp. 25-50.

[45] E.N. Frankel, R.F. Garwood, B.P.S. Khambay, G.P. Moss, B.C.L. Weedon, Stereochemistry of olefin and fatty-acid oxidation .3. The allylic hydroperoxides from the autoxidation of methyl oleate, Journal of the Chemical Society-Perkin Transactions 1, (10) (1984) 2233-2240.

[46] E.N. Frankel, R.F. Garwood, J.R. Vinson, B.C.L. Weedon, Stereochemistry of olefin and fatty-acid oxidation .1. Autoxidation of hexene and hepta-2,5-diene isomers, Journal of the Chemical Society-Perkin Transactions 1, (11) (1982) 2707-2713.

[47] A.C. Rustan, C.A. Drevon, Fatty Acids: Structures and Properties, in: J.W. Sons (Ed.) Encyclopedia of Life Sciences, 2005, pp. 1-7.

[48] E.N. Frankel, Hydroperoxides, in: H.W. Schultz, E.A. Day, R.O. Sinnhuber (Eds.) Symposium on Foods: Lipids and Their Oxidation, Avi Publishing, Westport, USA, 1962, pp. 51-78.

[49] S.H. Fatemi, E.G. Hammond, Analysis of oleate, linoleate and linolenate hydroperoxides in oxidized ester mixtures, Lipids, 15(5) (1980) 379-385.

[50] P. Bouchon, D.L. Pyle, Modeling oil absorption during post-frying cooling. II: Solution of the Mathematical Model, Model Testing and Simulations, Food and Bioproducts Processing, 83(4) (2005) 261-272.

[51] O.O. Fasina, Z. Colley, Viscosity and Specific Heat of Vegetable Oils as a Function of Temperature: $35^{\circ} \mathrm{C}$ to $180^{\circ} \mathrm{C}$, International Journal of Food Properties, 11(4) (2008) 738-746. [52] E.E.G. Rojas, J.S.R. Coimbra, J. Telis-Romero, Thermophysical Properties of Cotton, Canola, Sunflower and Soybean Oils as a Function of Temperature, International Journal of Food Properties, 16(7) (2013) 1620-1629.

[53] B. Eyglunent, Manuel de thermique: théorie et pratique., 2nd ed., Hermès-Lavoisier, 2003. [54] S. Globe, D. Dropkin, Natural-Convection Heat Transfer in Liquids Confined by Two Horizontal Plates and Heated From Below, Journal of Heat Transfer, 81(1) (1959) 24-28.

[55] T. Hiroaki, M. Hiroshi, Turbulent natural convection in a horizontal water layer heated from below, International Journal of Heat and Mass Transfer, 23(9) (1980) 1273-1281.

[56] M.T. Morales, R. Przybylski, Olive Oil Oxidation, in: J. Harwood, R. Aparicio (Eds.) Handbook of Olive Oil: Analysis and Properties, Springer US, Boston, MA, 2000, pp. 459-490. [57] M. Tokita, Morita, M., Different Rates of Geometric Isomers of Linoleate Hydroperoxide in Acid-catalyzed Decomposition, Agricultural and Biological Chemistry, 49(12) (1985) 35453550.

[58] S. Bergström, Autoxidation of Linoleic Acid, Nature, 156(3972) (1945) 717-718.

[59] B. Bozan, F. Temelli, Chemical composition and oxidative stability of flax, safflower and poppy seed and seed oils, Bioresource Technology, 99 (2008) 6354-6359.

[60] J. Mallégol, J.-L. Gardette, J. Lemaire, Long-term behavior of oil-based varnishes and paints. Photo- and thermooxidation of cured linseed oil, Journal of the American Oil Chemists' Society, 77(3) (2000) 257-263. 
[61] J. Mallégol, J.L. Gardette, J. Lemaire, Long-term behavior of oil-based varnishes and paints. Fate of hydroperoxides in drying oils, Journal of the American Oil Chemists Society, 77(3) (2000) 249-255.

948 [62] J. Mallégol, L. Gonon, S. Commereuc, V. Verney, Thermal (DSC) and chemical (iodometric titration) methods for peroxides measurements in order to monitor drying extent of alkyd resins, Progress in Organic Coatings, 41(1) (2001) 171-176. Autoxydation von Estern ungesättigter Fettsäuren 5. Mitt. Über den Mechanismus des Hydroperoxidabbaus, Food / Nahrung, 17(3) (1973) 313-322.

[64] A. Hopia, S.W. Huang, E.N. Frankel, Effect of alpha-tocopherol and trolox on the decomposition of methyl linoleate hydroperoxides, Lipids, 31(4) (1996) 357-365.

[65] M. Natake, G.-i. Danno, Antioxidative Action of Methionine in the Autoxidation of Linoleic Acid, Eiyo To Shokuryo, 26(4) (1973) 245-250.

958 [66] M.-E. Cuvelier, Boussard, A., Keller, S., Golitin, L., Roux, S., Kinetic modelling of hydroperoxide degradation amplified by Fenton reactions in a model of edible oils., in: Engineering Future Food, Milano, 2017, pp. 1.

[67] B. Castaing, G. Gunaratne, F. Heslot, L. Kadanoff, A. Libchaber, S. Thomae, W.-Z. Wu, S. Zaleski, G. Zanetti, Scaling of hard thermal turbulence in Rayleigh-Bénard convection, Journal of Fluid Mechanics, 204 (2006) 1-30.

964

965

966

967

968

969

970

971

972

973

[68] M. Kaczorowski, C. Wagner, Analysis of the thermal plumes in turbulent Rayleigh-Bénard convection based on well-resolved numerical simulations, Journal of Fluid Mechanics, 618 (2009) 89-112.

[69] A.M. Obukhov, Some specific features of atmospheric turbulence, Journal of Geophysical Research (1896-1977), 67(8) (1962) 3011-3014.

[70] R. Bolgiano Jr., Turbulent spectra in a stably stratified atmosphere, Journal of Geophysical Research (1896-1977), 64(12) (1959) 2226-2229.

[71] A. Basu, J.K. Bhattacharjee, Kolmogorov or Bolgiano-Obukhov scaling: Universal energy spectra in stably stratified turbulent fluids, Physical Review E, 100(033119) (2019) 1-8.

[72] F.X. Trias, D. Folch, A. Gorobets, A. Oliva, Spectrally-Consistent Regularization of Navier-Stokes Equations, Journal of Scientific Computing, 79(2) (2019) 992-1014.

[73] P.K. Kundu, I.M. Cohen, D.R. Dowling, Fluid Mechanics, 6th ed., Elsevier, London, UK, 2015.

[74] P.G. Mestayer, C.H. Gibson, M.F. Coantic, A.S. Patel, Local anisotropy in heated and cooled turbulent boundary layers, The Physics of Fluids, 19(9) (1976) 1279-1287.

[75] C.H. Gibson, C.A. Friehe, S.O. McConnell, Structure of sheared turbulent fields, The Physics of Fluids, 20(10) (1977) S156-S167.

[76] M. Holzer, E.D. Siggia, Turbulent mixing of a passive scalar, Physics of Fluids, 6(5) (1994) 1820-1837.

[77] A. Pumir, A numerical study of the mixing of a passive scalar in three dimensions in the presence of a mean gradient, Physics of Fluids, 6(6) (1994) 2118-2132.

[78] R. Metzler, J.-H. Jeon, A.G. Cherstvy, E. Barkai, Anomalous diffusion models and their properties: non-stationarity, non-ergodicity, and ageing at the centenary of single particle tracking, Physical Chemistry Chemical Physics, 16(44) (2014) 24128-24164.

[79] J.A. Howard, K.U. Ingold, Absolute rate constants for hydrocarbon autoxidation. VI. Alkyl aromatic and olefinic hydrocarbons, Canadian Journal of Chemistry, 45(8) (1967) 793-802.

[80] A.J. Stirton, J. Turer, R.W. Riemenschneider, Oxygen Absorption of Methyl Esters of Fat Acids, and the Effect of Antioxidants, Journal of the American Oil Chemists Society, 22(4) (1945) 81-83. 
a.

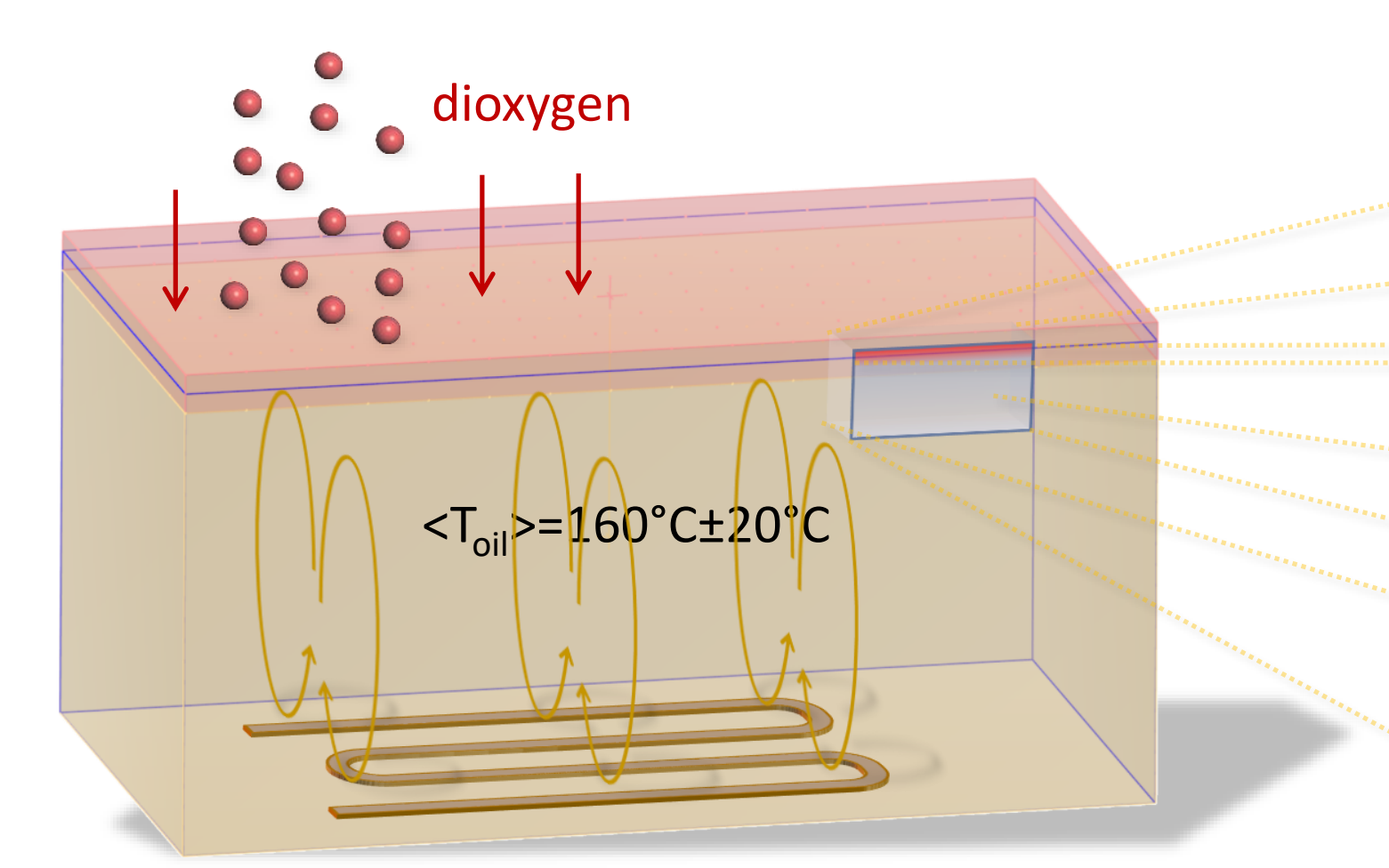

deep-fryer bath

b.

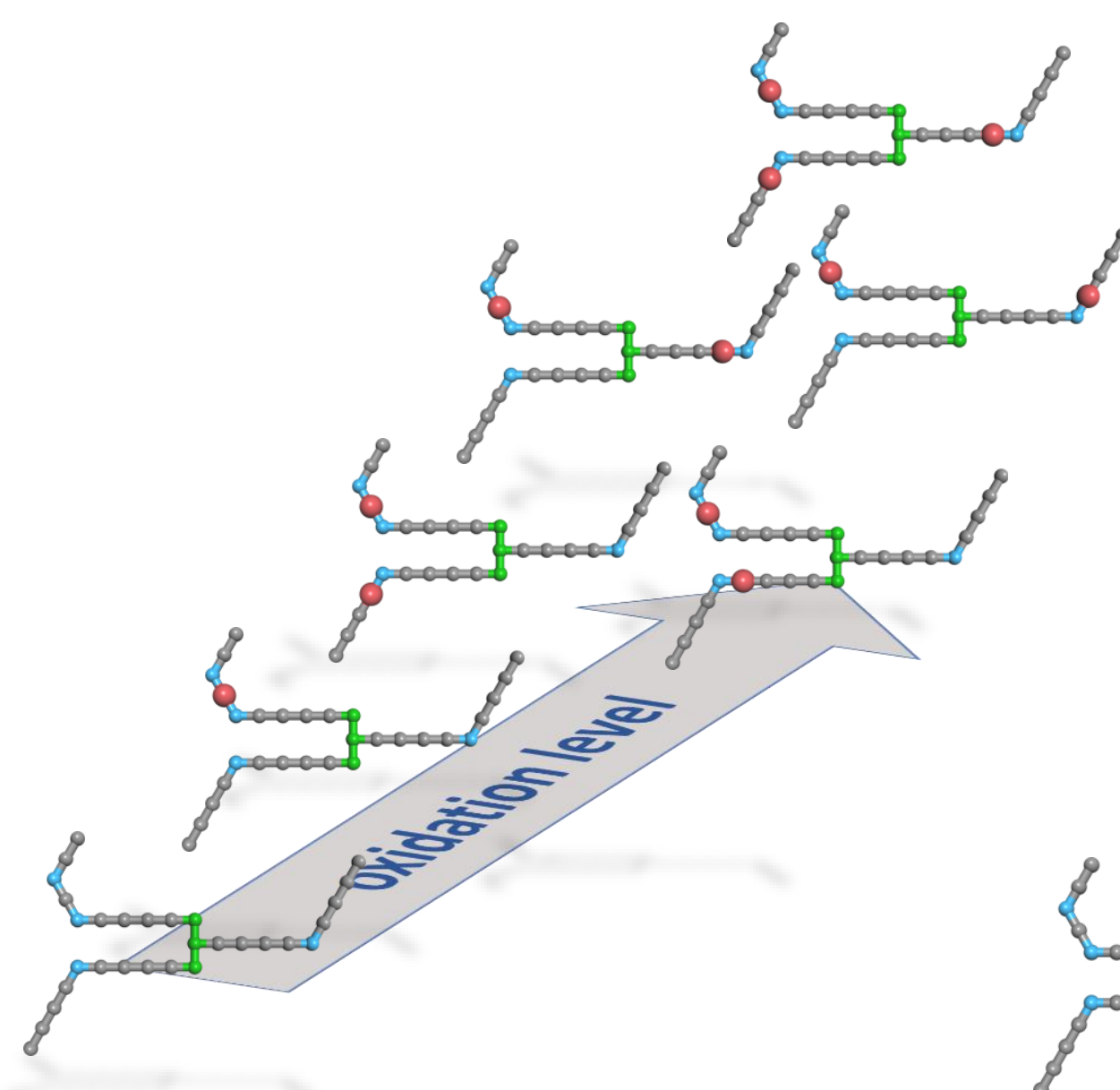

elementary volume at the extreme surface

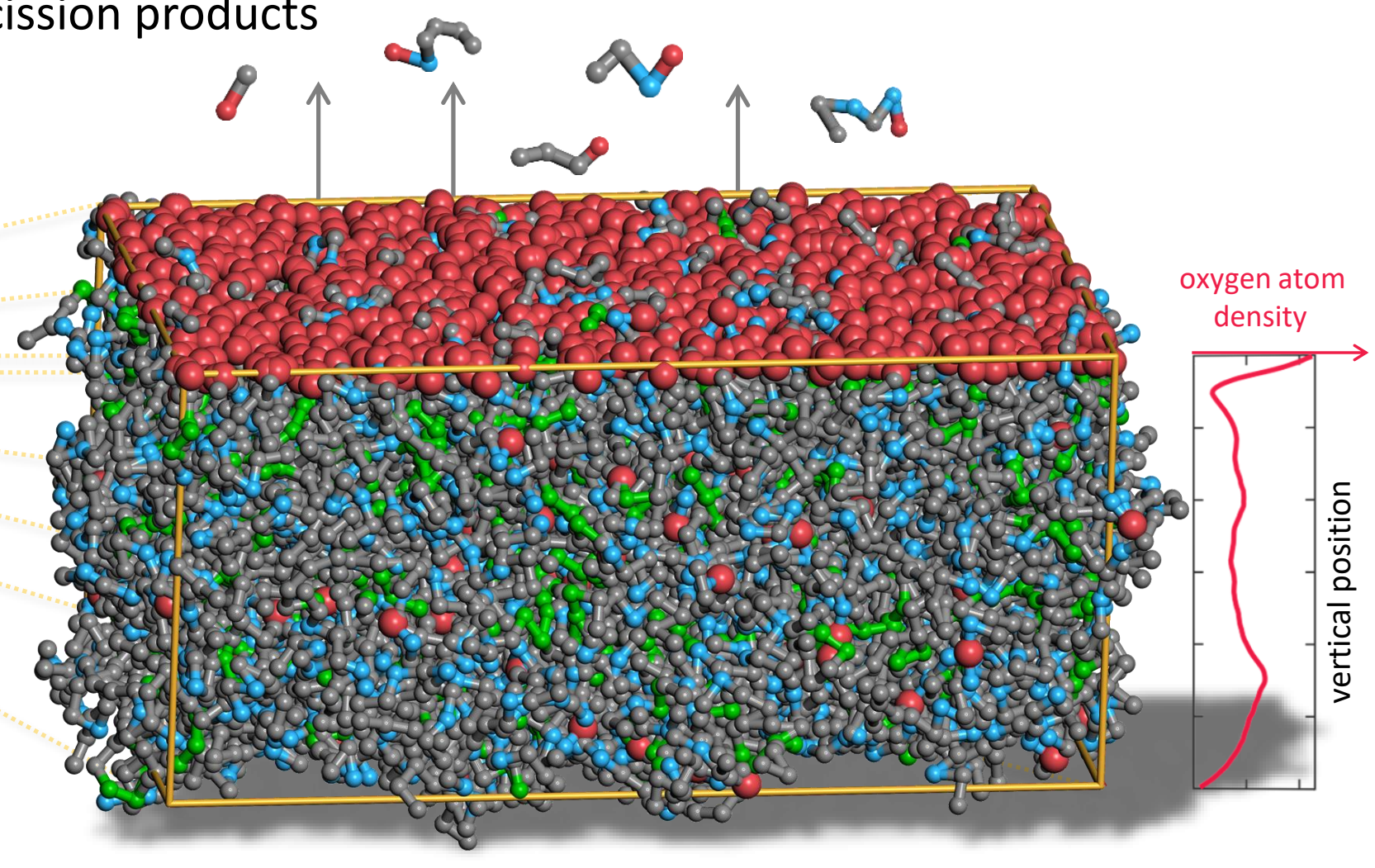

bead approximately 2:1 heavy atoms

ethyl-group

ethylene- group

ester group (on glycerol backbone)

ester, aldehyde, ketone after scission

hydroperoxide group or atmospheric dic

C.

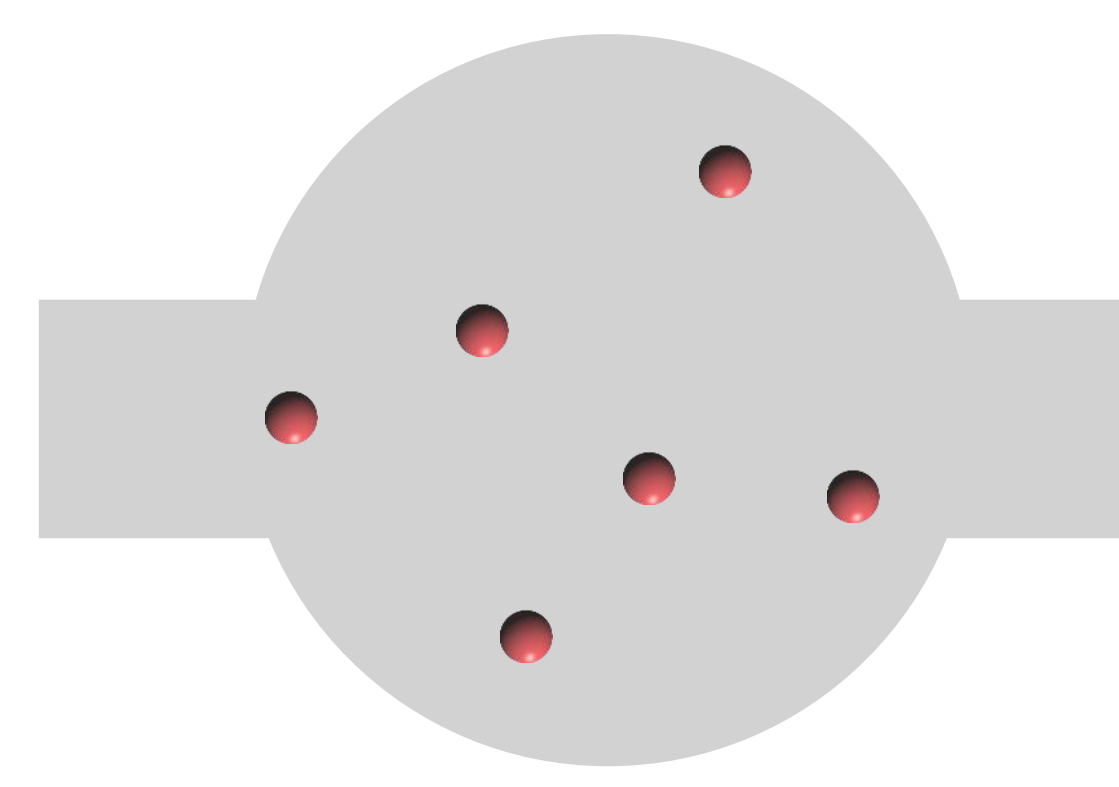

balance in hydroperoxide chemical functions or equivalent $\Upsilon_{2}$

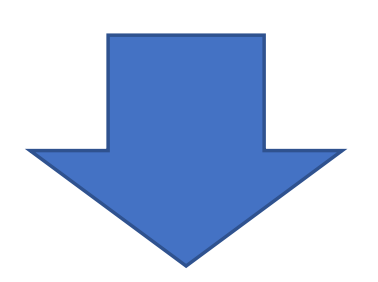

- balance of labile hydrogens

- average reactivity

- no specific thermal activation

- no effect of TAG composition

- no ability to predict the profile of oxidation products

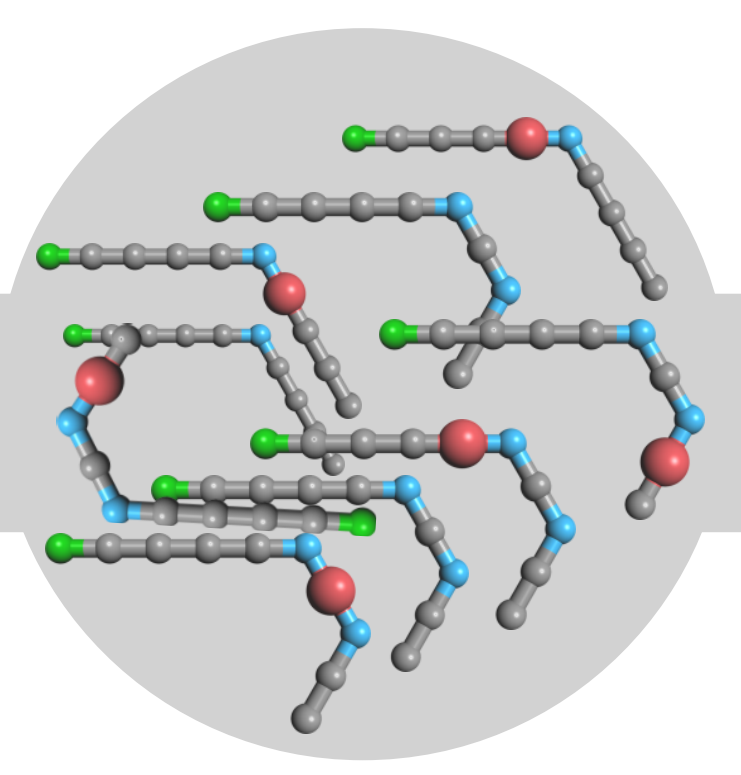

balance in fatty esters (FE)

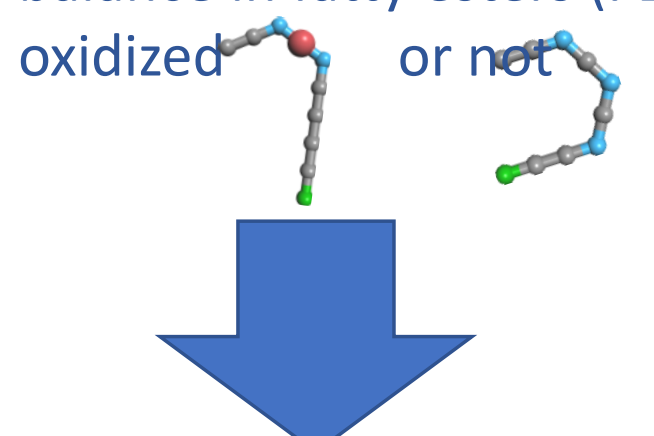

- specific reactivity based on FE composition

- specific thermal activation

- effect of TAG composition

- lineage of oxidation products

- no correlated transport for FE attached to the same glycerol backbone

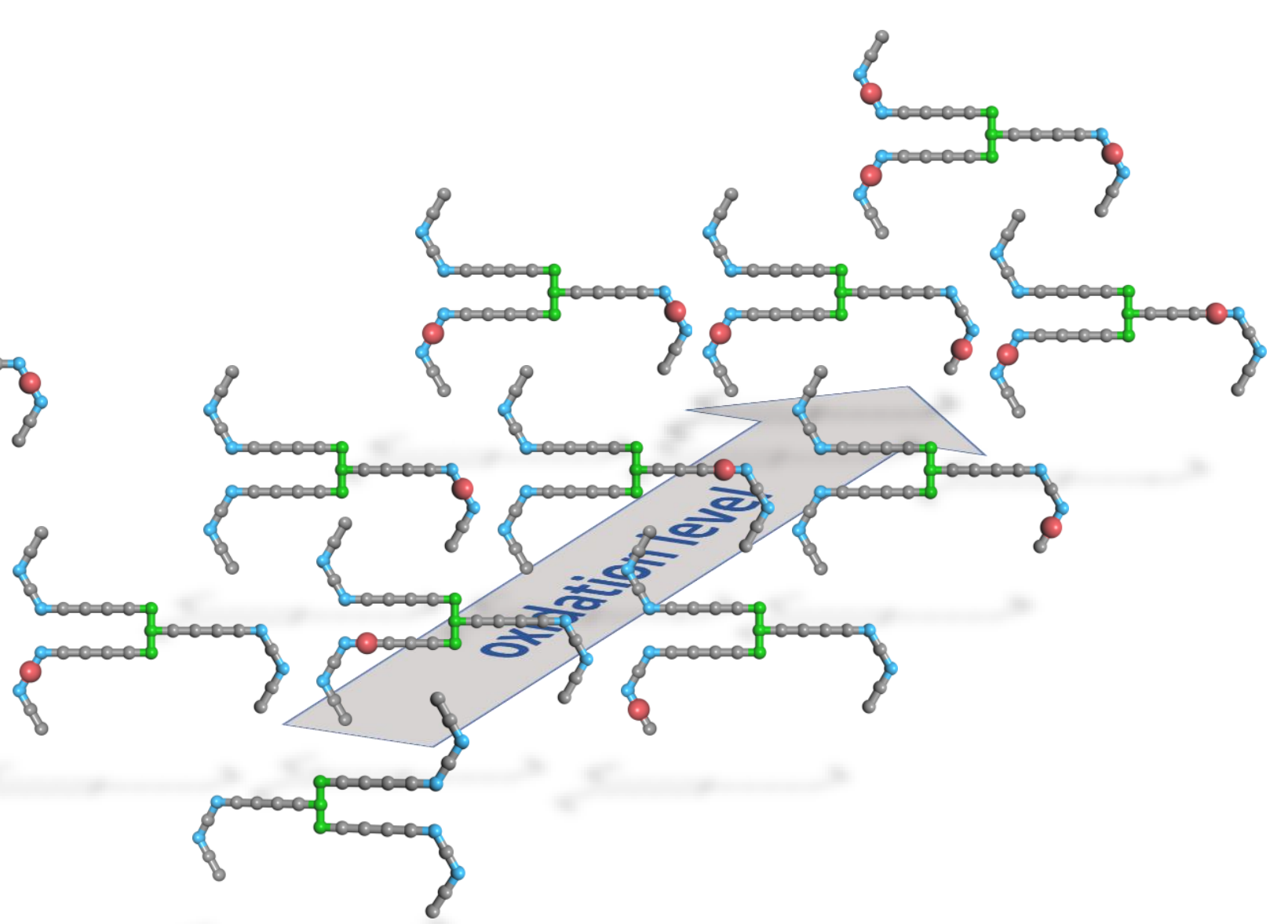

oxidability and prevalence in sunflower oil

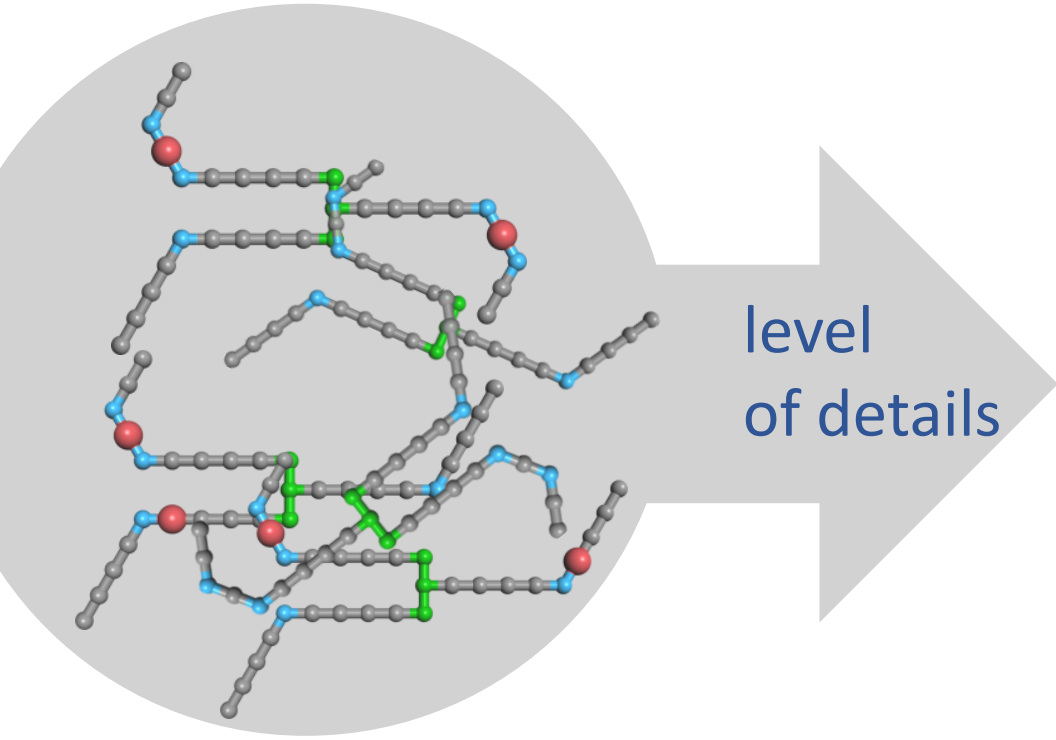

balance in triacylglycerols (TAG) oxidized

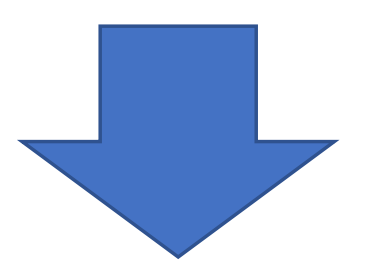

- realistic description

- correlated transport of FE attached to the same glycerol

- profile of oxidized TAG.

- possibility to add mono- and diacylglycerols. 


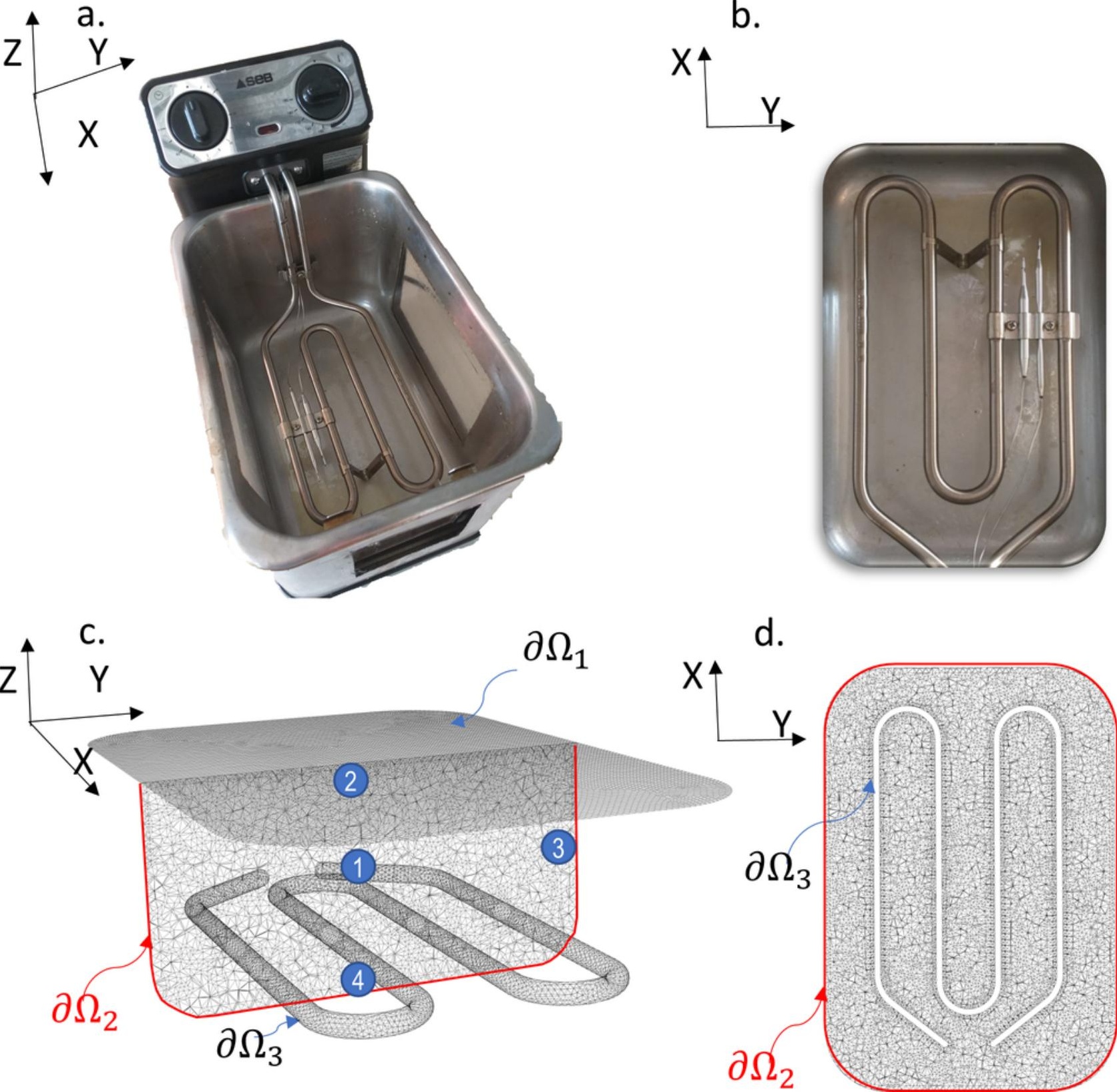




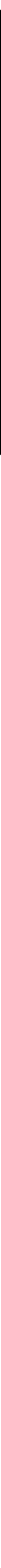




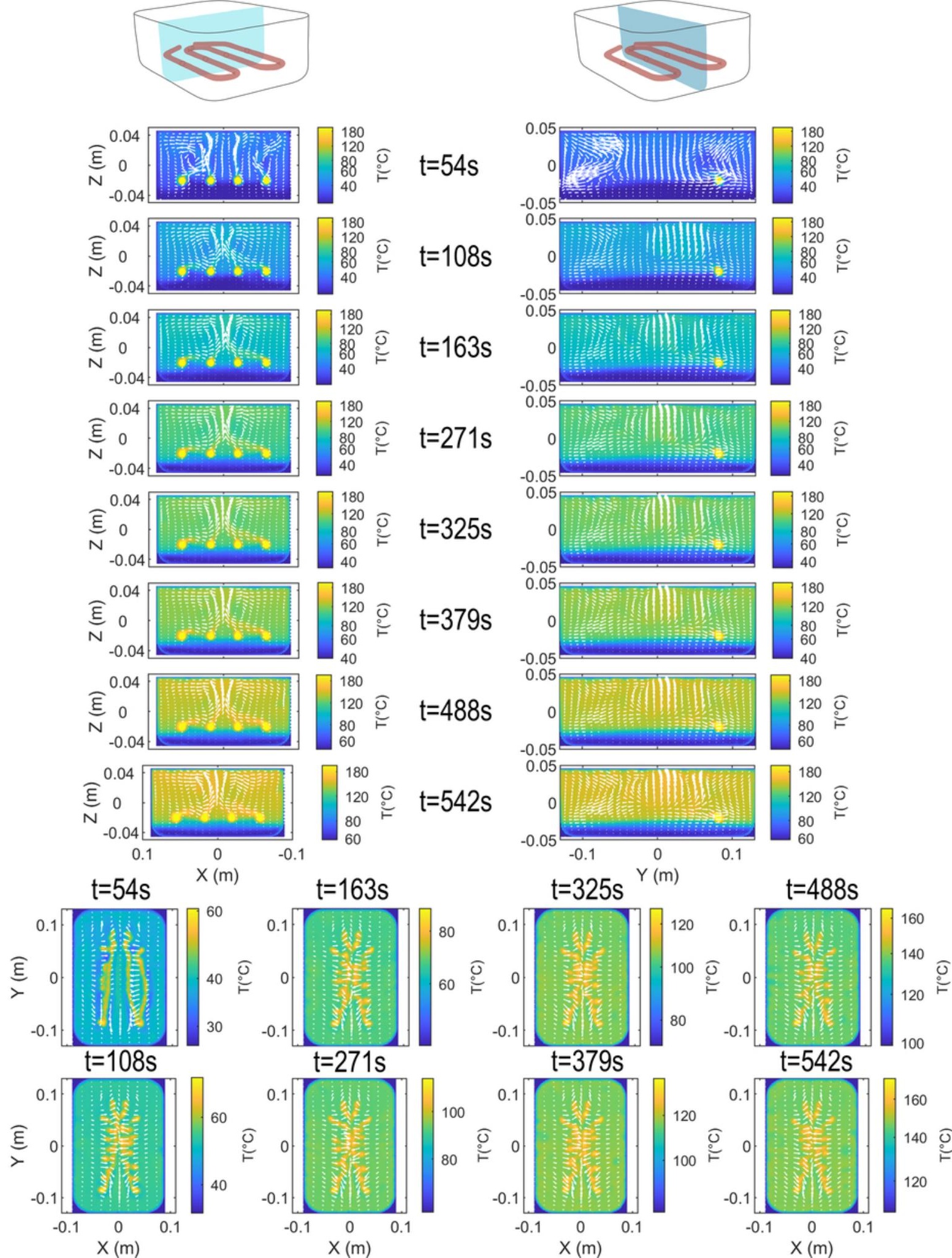




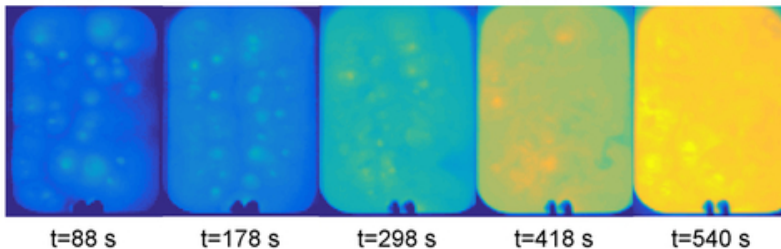

$\mathrm{T}\left({ }^{\circ} \mathrm{C}\right)$
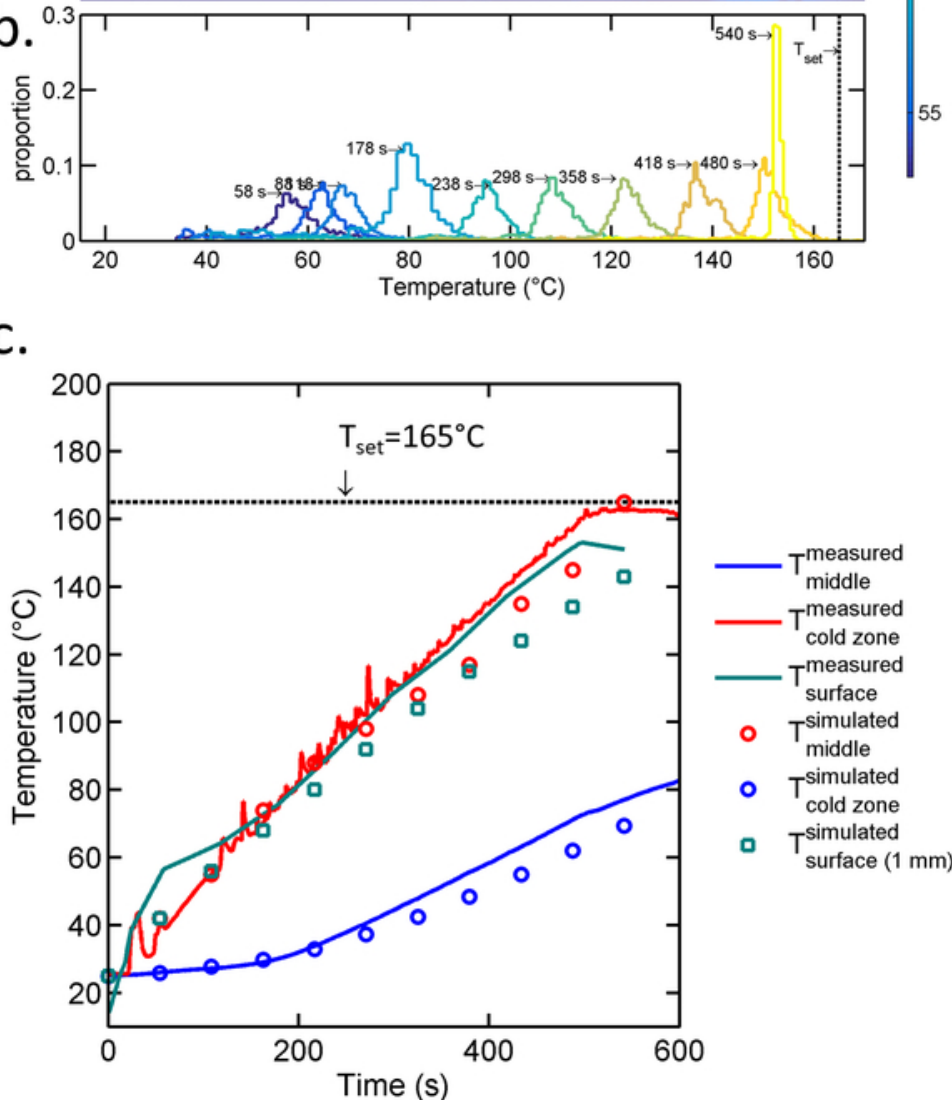


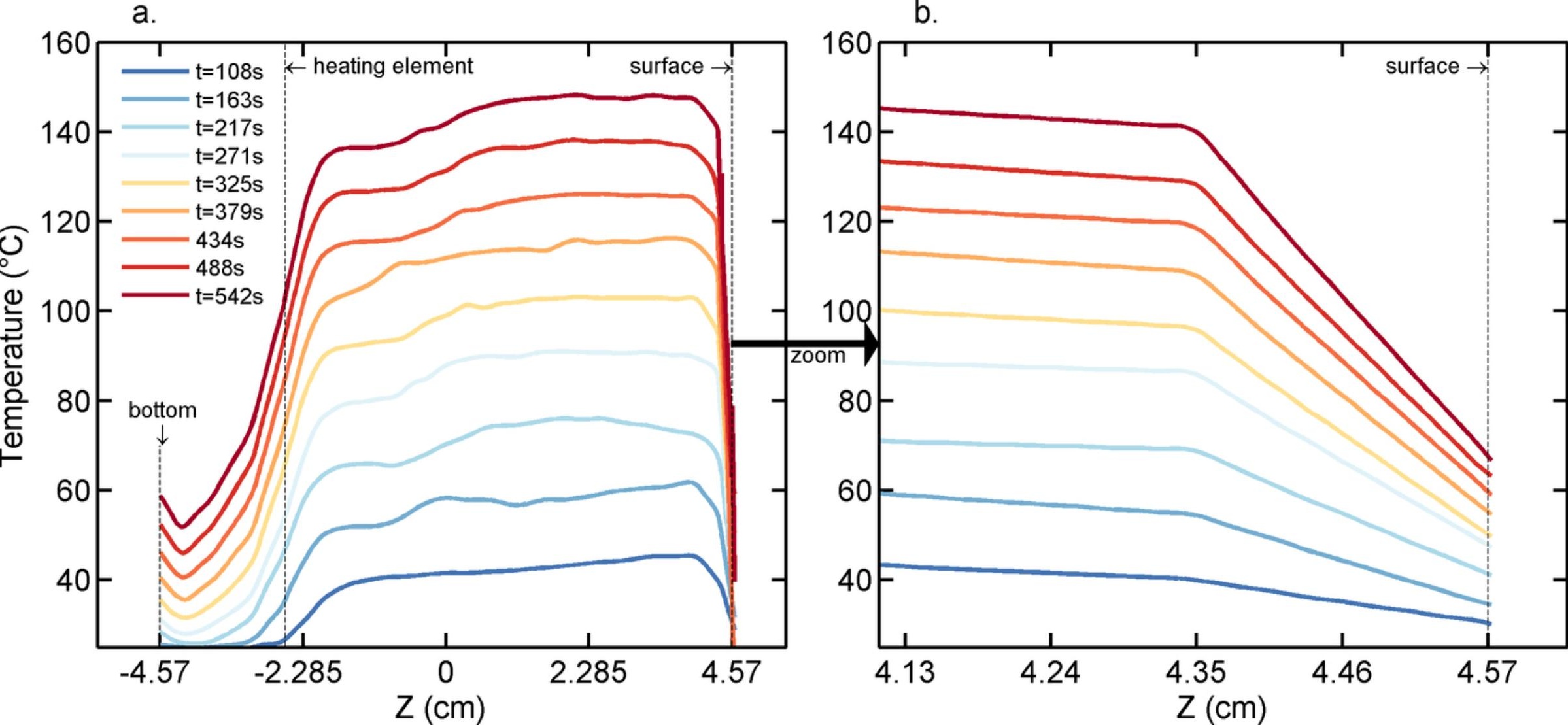



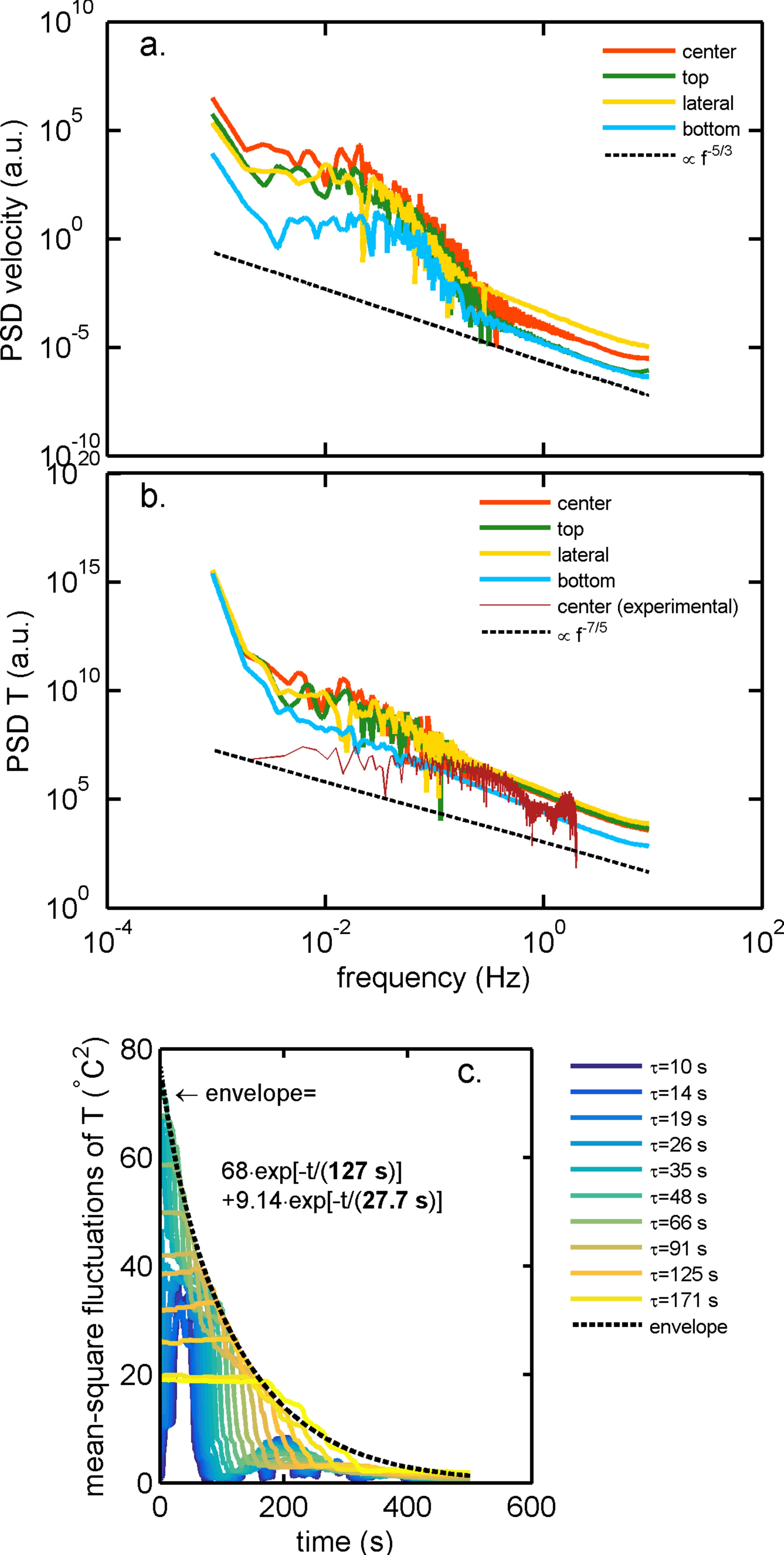
a.
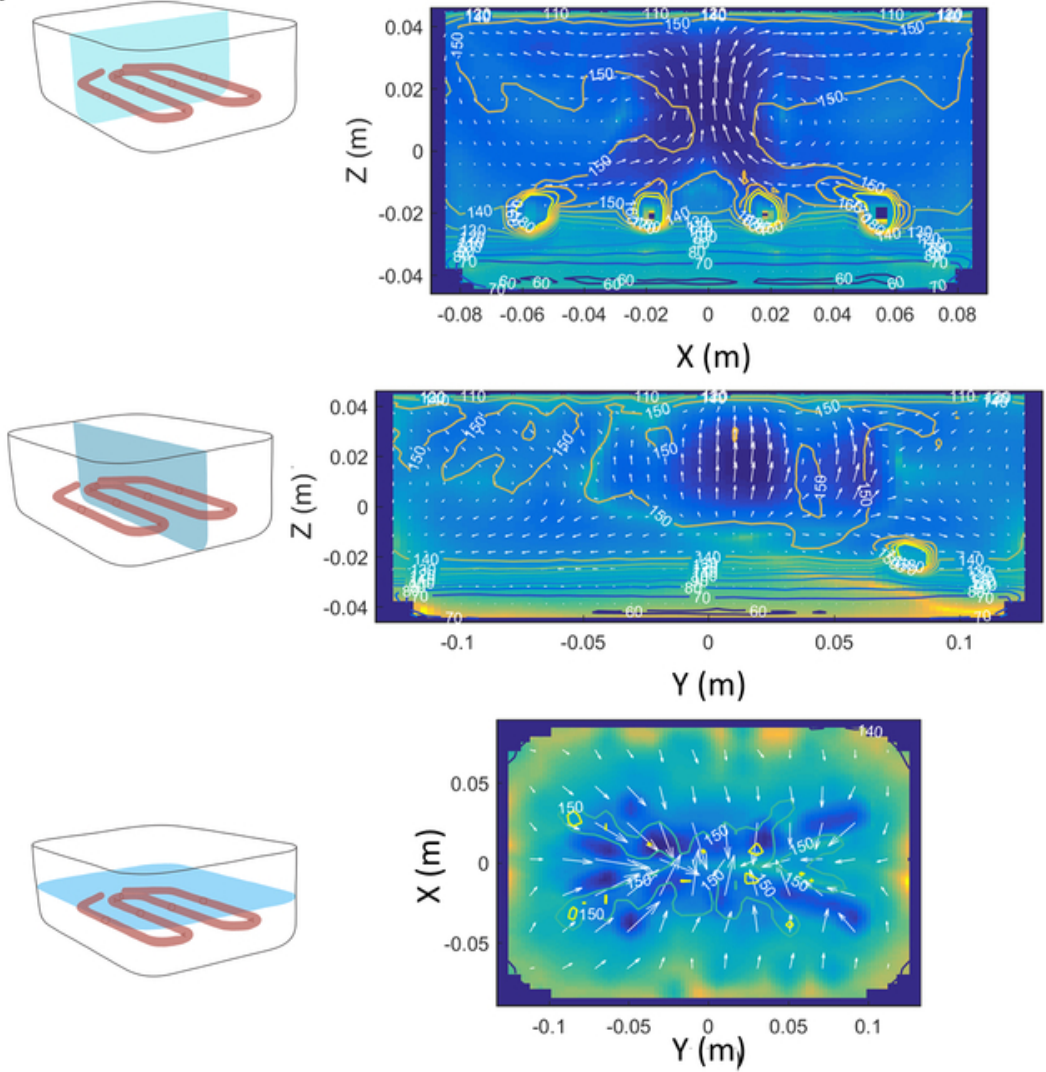

b.
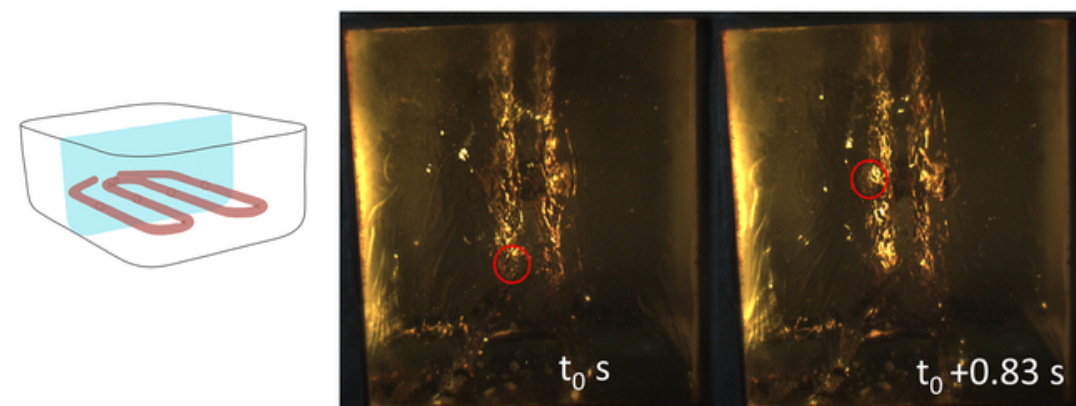

$\mathrm{t}_{\mathrm{r}}(\mathrm{s})$

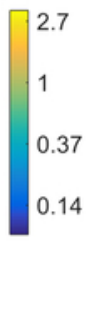

$t_{r}(s)$

$3 \cdot 10^{3}$
$4 \cdot 10^{2}$
55
7.4
1
0.14

$t_{r}(s)$

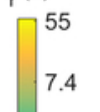

0.14

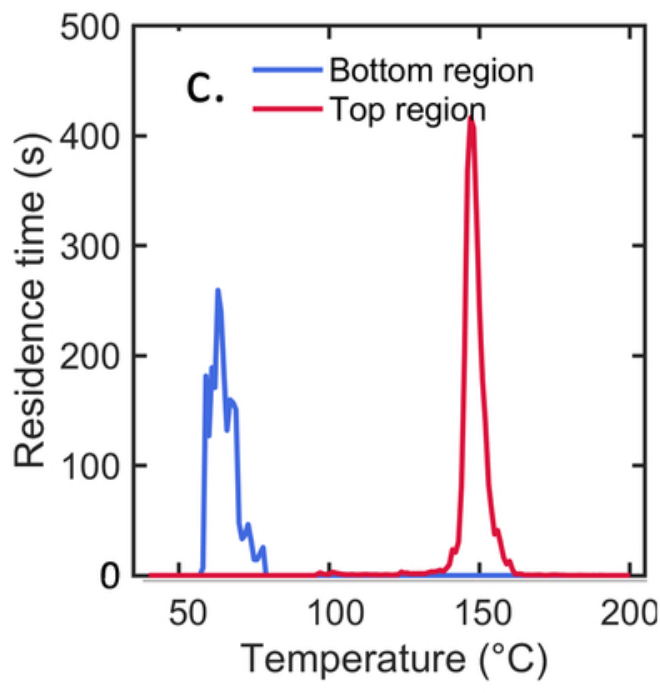

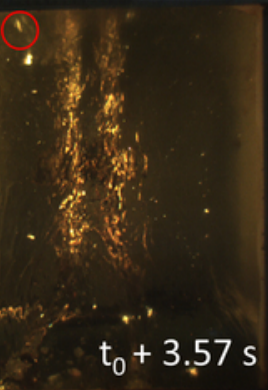
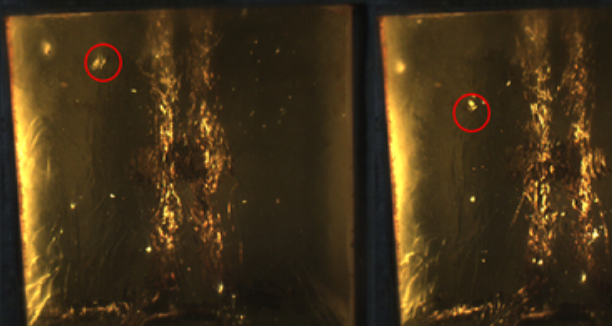

$t_{0}+5.47 \mathrm{~s}$

$t_{0}+6.17 \mathrm{~s}$
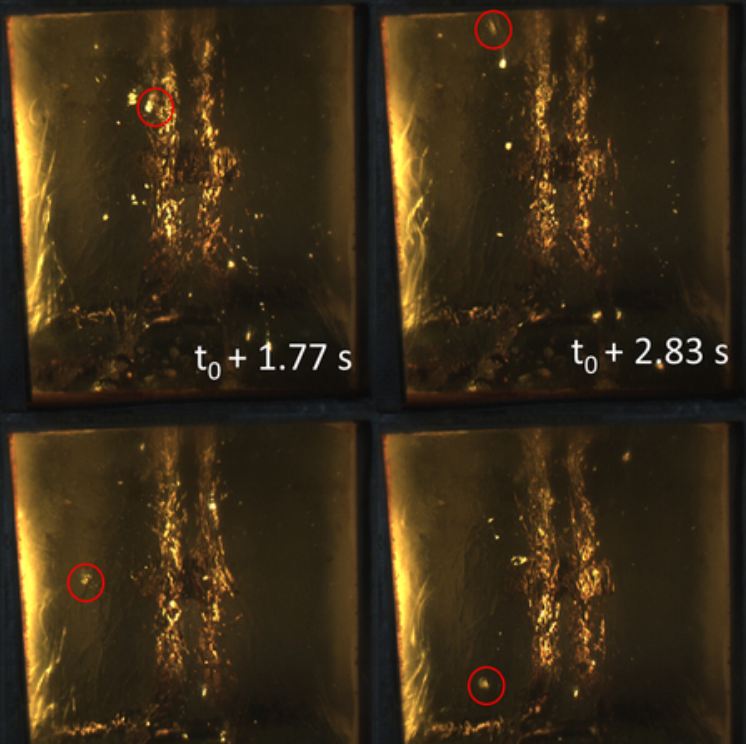

$t_{0}+9.37 \mathrm{~s}$

$t_{0}+10.47 \mathrm{~s}$ 

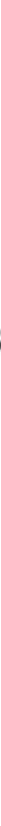

c.
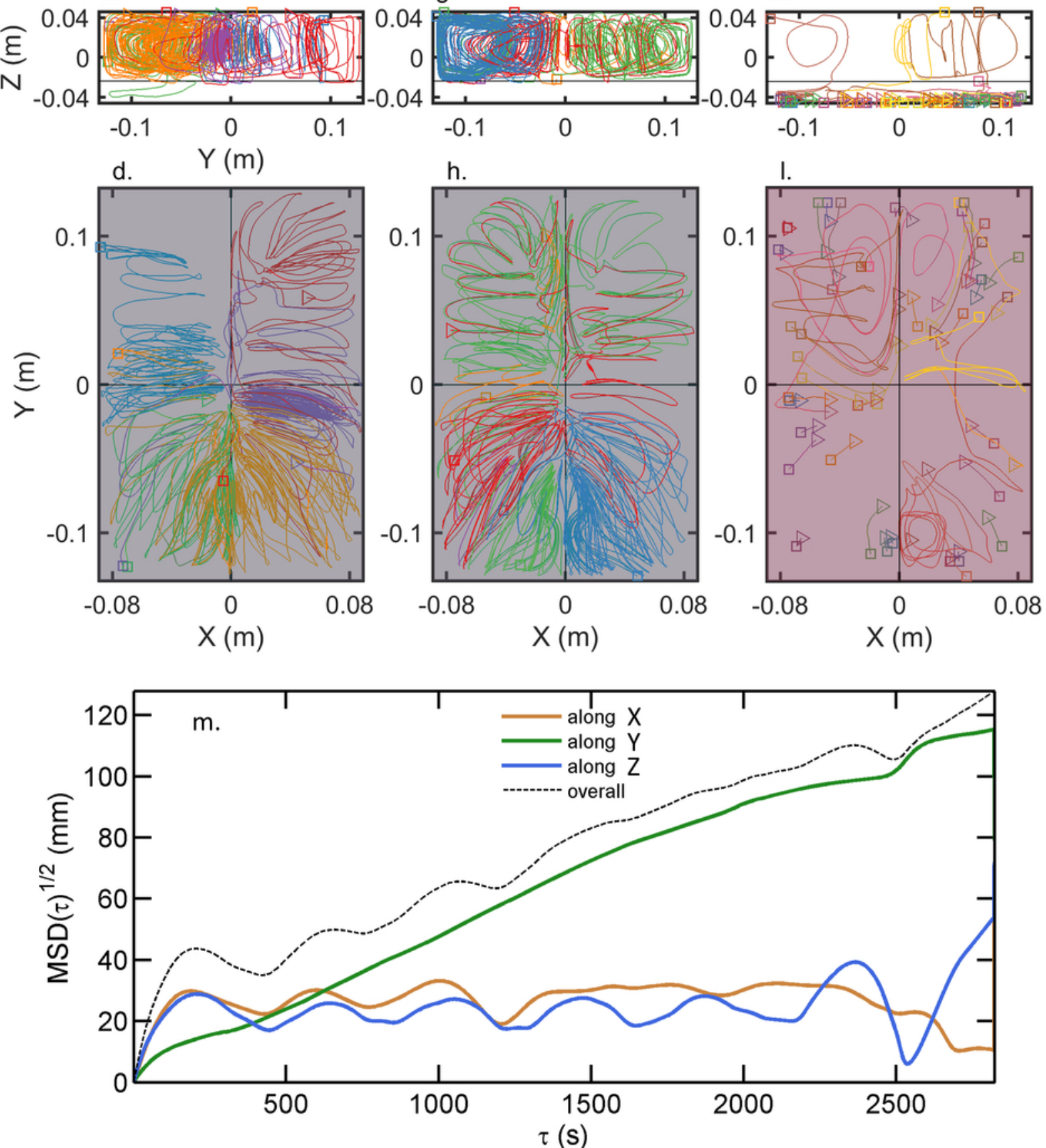


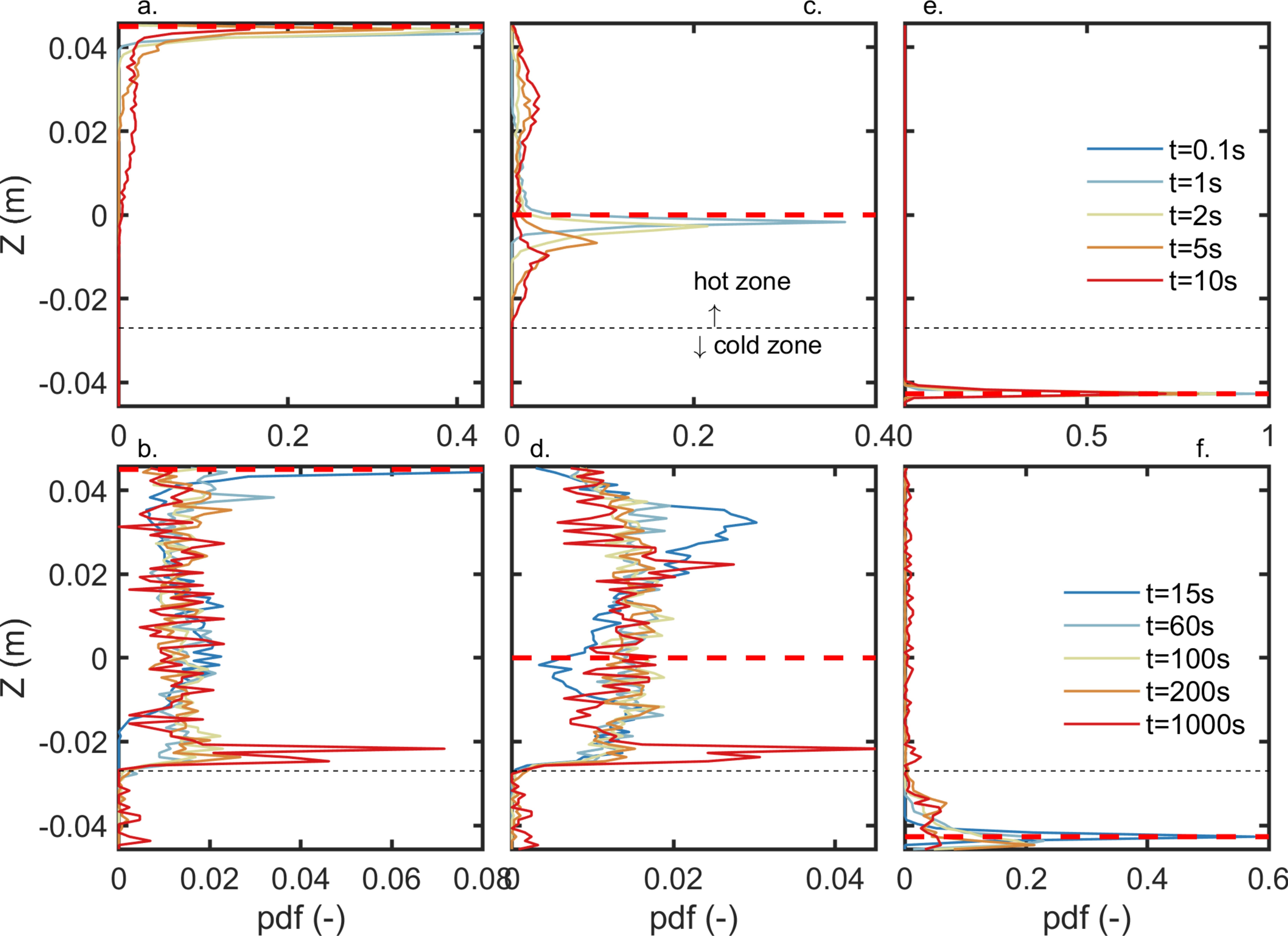




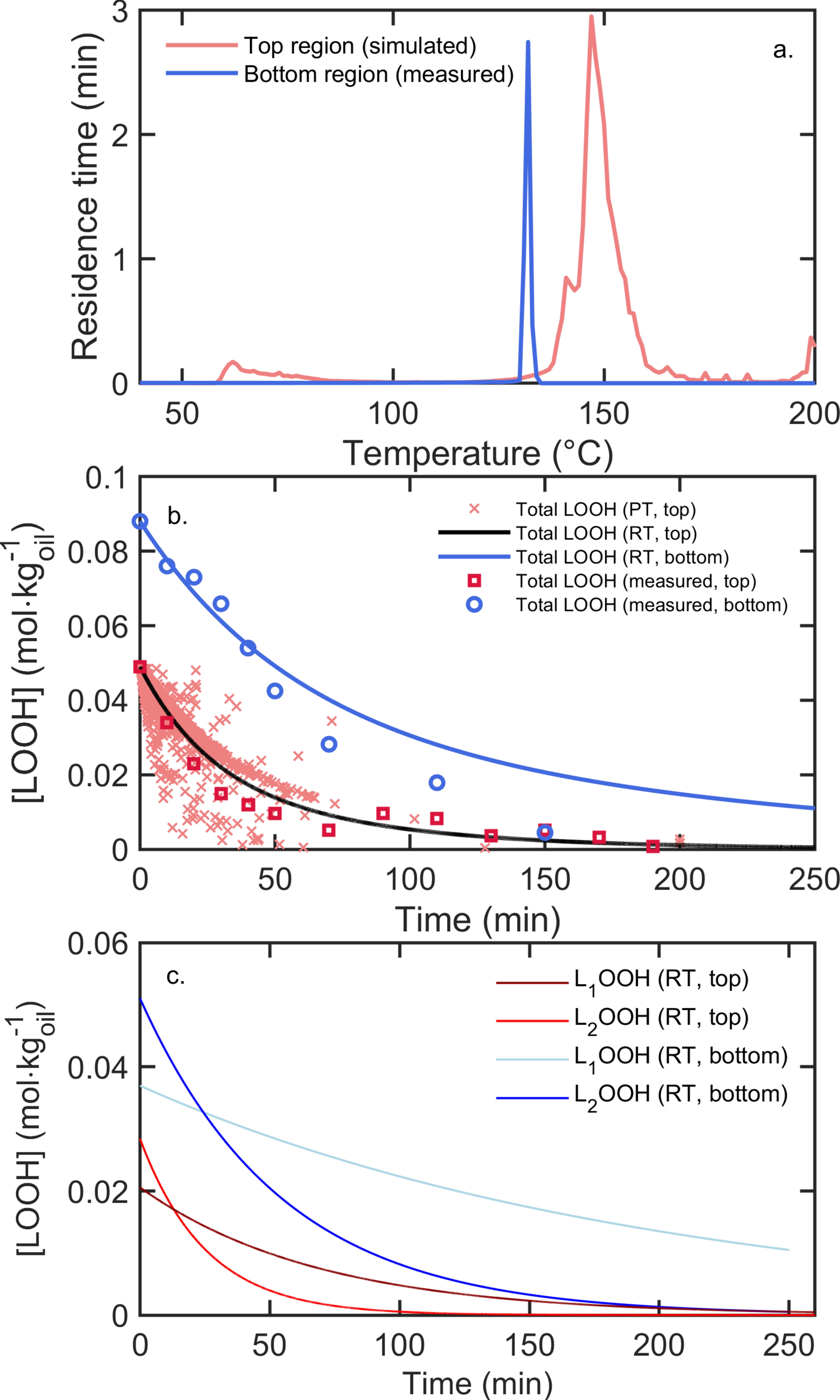


